\title{
Near-Infrared Photometry and Spectroscopy of $L$ and T Dwarfs: The Effects of Temperature, Clouds, and Gravity
}

\section{Citation}

Knapp, G. R., S. K. Leggett, X. Fan, M. S. Marley, T. R. Geballe, D. A. Golimowski, D. Finkbeiner, et al. 2004. "Near-Infrared Photometry and Spectroscopy of $L$ and T Dwarfs: The Effects of Temperature, Clouds, and Gravity." The Astronomical Journal 127 (6) (June): 3553-3578. doi:10.1086/420707.

\section{Published Version}

doi:10.1086/420707

\section{Permanent link}

http://nrs.harvard.edu/urn-3:HUL.InstRepos:33461894

\section{Terms of Use}

This article was downloaded from Harvard University's DASH repository, and is made available under the terms and conditions applicable to Other Posted Material, as set forth at http:// nrs.harvard.edu/urn-3:HUL.InstRepos:dash.current.terms-of-use\#LAA

\section{Share Your Story}

The Harvard community has made this article openly available.

Please share how this access benefits you. Submit a story.

Accessibility 


\title{
NEAR-INFRARED PHOTOMETRY AND SPECTROSCOPY OF L AND T DWARFS: THE EFFECTS OF TEMPERATURE, CLOUDS, AND GRAVITY
}

\author{
G. R. Knapp, ${ }^{1}$ S. K. Leggett, ${ }^{2}$ X. Fan, ${ }^{3}$ M. S. Marley, ${ }^{4}$ T. R. Geballe, ${ }^{5}$ D. A. Golimowski, ${ }^{6}$ D. Finkbeiner, ${ }^{1}$ J. E. Gunn, ${ }^{1}$ \\ J. Hennawi, ${ }^{1}$ Z. Ivezić, ${ }^{1}$ R. H. Lupton, ${ }^{1}$ D. J. Schlegel, ${ }^{1}$ M. A. Strauss, ${ }^{1}$ Z. I. Tsvetanov, ${ }^{6}$ K. Chiu, ${ }^{6}$ \\ E. A. Hoversten, ${ }^{6}$ K. Glazebrook, ${ }^{6}$ W. Zheng, ${ }^{6}$ M. Hendrickson, ${ }^{6}$ C. C. Williams, ${ }^{6}$ A. Uomoto, ${ }^{6,7}$ \\ F. J. Vrba, ${ }^{8}$ A. A. Henden, ${ }^{8,9}$ C. B. Luginbuhl, ${ }^{8}$ H. H. Guetter, ${ }^{8}$ J. A. Munn, ${ }^{8}$ B. Canzian, ${ }^{8}$ \\ Donald P. SchneIder, ${ }^{10}$ AND J. BRINKMANN ${ }^{11}$ \\ Received 2003 November 10; accepted 2004 February 18
}

\begin{abstract}
We present new $J H K$ photometry on the MKO-NIR system and $J H K$ spectroscopy for a large sample of L and $\mathrm{T}$ dwarfs. Photometry has been obtained for 71 dwarfs, and spectroscopy for 56 . The sample comprises newly identified very red objects from the Sloan Digital Sky Survey (SDSS) and known dwarfs from the SDSS and the Two Micron All Sky Survey (2MASS). Spectral classification has been carried out using four previously defined indices from Geballe et al. that measure the strengths of the near infrared water and methane bands. We identify nine new L8-9.5 dwarfs and 14 new T dwarfs from SDSS, including the latest yet found by SDSS, the T7 dwarf SDSS J175805.46+463311.9. We classify 2MASS J04151954-0935066 as T9, the latest and coolest dwarf found to date. We combine the new results with our previously published data to produce a sample of $59 \mathrm{~L}$ dwarfs and $42 \mathrm{~T}$ dwarfs with imaging data on a single photometric system and with uniform spectroscopic classification. We compare the near-infrared colors and absolute magnitudes of brown dwarfs near the L-T transition with predictions made by models of the distribution and evolution of photospheric condensates. There is some scatter in the Geballe et al. spectral indices for L dwarfs, suggesting that these indices are probing different levels of the atmosphere and are affected by the location of the condensate cloud layer. The near-infrared colors of the L dwarfs also show scatter within a given spectral type, which is likely due to variations in the altitudes, spatial distributions, and thicknesses of the clouds. We have identified a small group of late-L dwarfs that are relatively blue for their spectral type and that have enhanced $\mathrm{FeH}, \mathrm{H}_{2} \mathrm{O}$, and $\mathrm{K}$ I absorption, possibly due to an unusually small amount of condensates. The scatter seen in the $H-K$ color for late-T dwarfs can be reproduced by models with a range in surface gravity. The variation is probably due to the effect on the $K$-band flux of pressure-induced $\mathrm{H}_{2}$ opacity. The correlation of $H-K$ color with gravity is supported by the observed strengths of the $J$-band $\mathrm{K}$ I doublet. Gravity is closely related to mass for field $\mathrm{T}$ dwarfs with ages greater than $10^{8} \mathrm{yr}$ and the gravities implied by the $H-K$ colors indicate that the T dwarfs in our sample have masses in the range $15-75 M_{\text {Jupiter }}$. One of the SDSS dwarfs, SDSS J111010.01+011613.1, is possibly a very low mass object, with $\log g \sim 4.2-4.5$ and mass $\sim 10-15 M_{\text {Jupiter }}$.
\end{abstract}

Key words: infrared: stars — stars: fundamental parameters — stars: late-type — stars: low-mass, brown dwarfs

\section{INTRODUCTION}

Since the discovery of dwarfs of spectral type later than M as companions to nearby stars (Becklin \& Zuckerman 1988; Nakajima et al. 1995) major observational and theoretical progress has been made, thanks to sensitive new wide-area surveys at optical $(0.4-1.0 \mu \mathrm{m})$ and infrared $(1.0-2.5 \mu \mathrm{m})$ wavelengths and models of atmospheres of dwarfs with effective temperatures between those of the coolest stars and the giant planets (see the reviews by Chabrier \& Baraffe 2000; Burrows et al. 2001). Two new spectral classes have been identified later than type M: the L dwarfs, characterized by the

\footnotetext{
${ }^{1}$ Princeton University Observatory, Peyton Hall, Princeton, NJ 085441001.

${ }^{2}$ United Kingdom Infrared Telescope, Joint Astronomy Centre, 660 North A'ohoku Place, Hilo, HI 96720.

${ }_{3}^{3}$ Steward Observatory, 933 North Cherry Avenue, Tucson, AZ 85721.

${ }^{4}$ NASA/Ames Research Center, Mail Stop 245-3, Moffett Field, CA 94035 .

${ }^{5}$ Gemini Observatory, 670 North A'ohoku Place, Hilo, HI 96720.

${ }^{6}$ Department of Physics and Astronomy, Johns Hopkins University, 3400 North Charles Street, Baltimore, MD 21218.
}

disappearance of gas-phase $\mathrm{TiO}$ and $\mathrm{VO}$, and the $\mathrm{T}$ dwarfs, characterized by methane-band absorption in the $H$ and $K$ spectral regions (Martín et al. 1997, 1999b; Kirkpatrick et al. 1999, 2000; Strauss et al. 1999; Leggett et al. 2000, 2002b; Geballe et al. 2002, hereafter G02; Burgasser et al. 2002a; Hawley et al. 2002). Most $\mathrm{L}$ dwarfs and all $\mathrm{T}$ dwarfs are brown dwarfs. These objects are of interest because they occupy the mass range between that of stars and giant planets; because many of them are likely to have the intrinsic properties of giant planets, which at present cannot be directly observed; and because they allow the investigation of the initial mass function to substellar masses.

\footnotetext{
${ }^{7}$ Carnegie Observatories, 813 Santa Barbara Street, Pasadena, California 91101.

${ }^{8}$ US Naval Observatory, Flagstaff Station, P.O. Box 1149, Flagstaff, AZ 86002-1149.

${ }^{9}$ Universities Space Research Association, 1101 17th Street, NW, Washington, DC 20036.

${ }^{10}$ Department of Astronomy and Astrophysics, 525 Davey Laboratory, Pennsylvania State University, University Park, PA 16802.

${ }^{11}$ Apache Point Observatory, 2001 Apache Point Road, P.O. Box 59, Sunspot, NM 88349.
} 
Field $\mathrm{L}$ and $\mathrm{T}$ dwarfs have been discovered in large numbers in recent sky surveys: the Deep Near Infrared Survey (DENIS, Epchtein 1997); the Two Micron All Sky Survey (2MASS, Skrutskie et al. 1997; Beichman et al. 1998), and the optical Sloan Digital Sky Survey (SDSS, York et al. 2000). Including objects described in the present paper, there are now about $280 \mathrm{~L}$ dwarfs and $58 \mathrm{~T}$ dwarf systems published (e.g., Delfosse et al. 1997, 1999; Kirkpatrick et al. 1999, 2000; Burgasser et al. 2002a, 2003e; G02). This large sample has been used to establish a complete spectral sequence from L0 to T9 (G02; Burgasser et al. 2002a; McLean et al. 2003; present paper).

Unlike stars, brown dwarfs lack a sustained source of thermonuclear energy and hence cool continuously, passing through the $\mathrm{L}$ and $\mathrm{T}$ stages, with their initial spectral types depending on their masses. Their observational properties are thus a function not only of mass and metallicity, but also of age. Not all dwarfs of a given spectral type or effective temperature are identical; they have different gravities and different colors, the latter likely due to differing amounts of particulate matter in the atmosphere. For example, in mid to late $\mathrm{L}$ dwarfs there is a large scatter in the $J H K$ colors and apparently no one-to-one correspondence between effective temperature and spectral type (Leggett et al. 2002a; Golimowski et al. 2004). Such considerations drive searches for and measurements of additional dwarfs, in order to more fully characterize their atmospheres.

2MASS and SDSS have been highly complementary in the discovery of $\mathrm{L}$ and $\mathrm{T}$ dwarfs. Most of the flux from late-type dwarfs is emitted longward of $1 \mu \mathrm{m}$, and the $J-H$ and $H-K$ colors of $\mathrm{M}$ and $\mathrm{L}$ dwarfs become redder with decreasing effective temperature, allowing the identification by $2 \mathrm{MASS}$ of large numbers of L dwarfs (Kirkpatrick et al. 1999, 2000). However, in the transition from $\mathrm{L}$ to $\mathrm{T}, \mathrm{CH}_{4}$ absorption appears in the $H$ and $K$ regions (and $\mathrm{H}_{2}$ absorption predominantly at $K$ ), strengthening with later spectral type and causing the $\mathrm{T}$ dwarfs to become increasingly blue in their $J H K$ colors. The $J H K$ colors of early-T dwarfs are similar to those of the common $\mathrm{K}$ and $\mathrm{M}$ stars, making their identification in 2MASS very difficult. However in the SDSS filters the dwarfs simply become redder and thus the early- $T$ dwarfs have been found primarily in SDSS imaging (Leggett et al. 2000). Only three objects with spectral types between T0 and T3.5 have been identified from sources other than the SDSS-Martín et al. (2001) in an optical and near-infrared imaging survey of the $\sigma$ Orionis cluster tentatively classified one member as T0, Liu et al. (2002) found a distant field T3-T4 dwarf in a deep $I z$ survey, and McCaughrean et al. (2004) found that $\epsilon$ Indi $\mathrm{B}$, discovered in a high proper motion optical survey, is a binary consisting of a T1 and T6 pair (see also Scholz et al. 2003, Smith et al. 2003, and Volk et al. 2003). To date, all $\mathrm{T}$ dwarfs later than T7 have been found in the 2MASS database (Burgasser et al. 2002a), but we expect such objects to be found in the SDSS imaging data as sky coverage is increased.

In this paper we present near-infrared photometry and spectra of new and previously reported $\mathrm{L}$ and $\mathrm{T}$ dwarfs (including 14 new $\mathrm{T}$ dwarfs) and compare the colors and spectra with predictions from state-of-the-art model atmospheres with and without clouds. The new objects observed are described in the next section, and the new $J H K$ photometric and spectroscopic observations are described in $\S 3$, where we also derive spectral types. Section 4 presents colors, spectral types, and absolute magnitudes for the entire body of near-infrared data on $\mathrm{L}$ and $\mathrm{T}$ dwarfs that we have accumulated to date. In $\S 5$ we compare these data with model atmospheres. The conclusions are given in $\S 6$.

\section{THE OBSERVED SAMPLE}

The general goals of our observational efforts are to identify samples of late $\mathrm{L}$ and $\mathrm{T}$ dwarfs that are at least representative and ideally complete, and to measure their spectral and photometric characteristics. To this end, we have observed new candidate very cool dwarfs selected from the photometric observations of the SDSS. We have also reobserved some previously published SDSS L and T dwarfs for which our observations are incomplete or suspect and have added some 2MASS dwarfs where they complement our sample. Previous observations of the 2MASS objects are discussed by Kirkpatrick et al. (1999, 2000), Reid et al. (2001), Burgasser et al. (2002a, 2002b, 2003b, 2003c), and Dahn et al. (2002).

Table 1 lists the names and SDSS $i, z$ photometry of 51 confirmed SDSS dwarfs, most of them previously unpublished. A small number of these dwarfs are also detected in the SDSS $r$ band, and these magnitudes are presented later in the paper. As noted in Table 1, a few of these objects are described by Hawley et al. (2002), who present optical spectral types of a large sample of $\mathrm{M}, \mathrm{L}$, and $\mathrm{T}$ dwarfs observed by SDSS. One object, 2MASS J090837.97+503208.0, was identified as an L dwarf by Cruz et al. (2003) from the 2MASS database while this paper was in preparation. Finding charts from the SDSS $z$-band imaging are presented in Figure 1 for all 51 objects in Table 1, as charts have not been previously published for any of the objects.

Following the IAU convention, the SDSS names are based on the J2000.0 coordinates at the epoch of the initial observation and will be abbreviated in the text when individual objects are discussed; thus, SDSS J003259.36+141036.6 will be called by the shortened name SDSS J0032+1410. A similar convention is used for 2MASS objects, whose full coordinate names are given later (in Table 9). Note that these faint stars and brown dwarfs are nearby and likely to have significant proper motions, so the names do not necessarily provide accurate coordinates for later epochs. A future paper will discuss the measured SDSS positions and proper motions, since many of the objects were measured at more than one epoch.

The SDSS $i$ and $z$ magnitudes are $\mathrm{AB}_{\nu}$ magnitudes, for which the zero point in all bands is 3631 Jy (Oke \& Gunn 1983; Fukugita et al. 1996). The ZJHK magnitudes used at the United Kingdom Infrared Telescope (UKIRT) and discussed in this paper are on the $m($ Vega $)=0.0$ system. The SDSS magnitudes are modified to be asinh magnitudes, identical to logarithmic magnitudes for high signal-to-noise ratio measurements (greater than $5 \sigma$ ) and linear with flux below this (Lupton, Gunn, \& Szalay 1999). For the observations discussed herein, zero flux density corresponds to $r=25.1$, $i=24.4$, and $z=22.8$.

The SDSS camera (Gunn et al. 1998) scans the sky and produces near-simultaneous CCD images in five filters covering the optical bands $u, g, r, i$, and $z$ (centered at 3551, 4686, 6166,7480 , and $8932 \AA$ ). The imaging data are reduced through a set of automated software pipelines. The photometric pipeline PHOTO (Lupton et al. 2003) corrects the data, finds and measures objects, and applies photometric and astrometric calibrations. The photometric calibration is provided via a network of standard stars (Hogg et al. 2001; Smith et al. 2002), and the astrometric calibration via matches to standard astrometric catalogs (Pier et al. 2003). The photometry is accurate to about $2 \%$ in $g, r$, and $i$, and to about $3 \%$ in $u$ and $z$ 
TABLE 1

New Confirmed Late M, L, and T Dwarfs from SDSS

\begin{tabular}{|c|c|c|c|c|}
\hline Object & $i$ & $\sigma_{i}$ & $z$ & $\sigma_{z}$ \\
\hline SDSS J000013.54+255418.6..... & 25.59 & 0.56 & 18.48 & 0.04 \\
\hline SDSS J000112.18+153535.5 .. & 20.29 & 0.04 & 18.55 & 0.03 \\
\hline SDSS J001608.44-004302.3.. & 21.11 & 0.11 & 19.34 & 0.07 \\
\hline 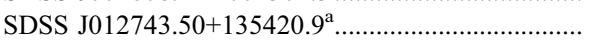 & 22.27 & 0.17 & 19.62 & 0.07 \\
\hline SDSS J020333.26-010812.5 ... & 22.76 & 0.36 & 20.36 & 0.15 \\
\hline SDSS J020735.60+135556.3 ${ }^{\mathrm{a}}$. & 19.84 & 0.03 & 18.06 & 0.02 \\
\hline 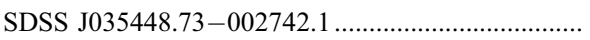 & 21.63 & 0.10 & 19.61 & 0.07 \\
\hline SDSS J040100.96-060933.0 .. & 22.93 & 0.49 & 20.19 & 0.20 \\
\hline 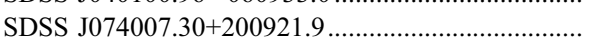 & 22.91 & 0.34 & 19.78 & 0.08 \\
\hline 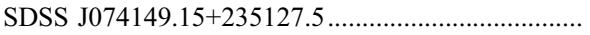 & 24.88 & 0.39 & 19.65 & 0.05 \\
\hline SDSS J074201.41+205520.5 ...... & 23.85 & 0.75 & 19.28 & 0.05 \\
\hline SDSS J074719.71+293748.6........ & 21.64 & 0.10 & 19.91 & 0.08 \\
\hline 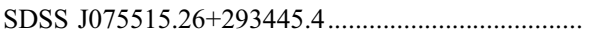 & 21.73 & 0.18 & 19.40 & 0.07 \\
\hline SDSS J075656.54+231458.5 ... & 22.89 & 0.31 & 19.82 & 0.07 \\
\hline 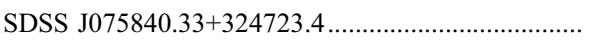 & 21.92 & 0.13 & 17.96 & 0.03 \\
\hline SDSS J080531.80+481233. $0^{\mathrm{a}} \ldots \ldots$. & 19.82 & 0.05 & 17.62 & 0.03 \\
\hline SDSS J080959.01+443422.2 ......... & 21.82 & 0.16 & 19.28 & 0.06 \\
\hline 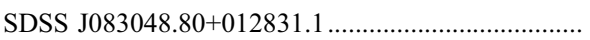 & 24.91 & 0.52 & 19.59 & 0.08 \\
\hline 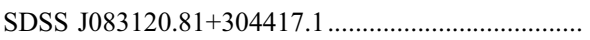 & 21.45 & 0.11 & 19.68 & 0.10 \\
\hline 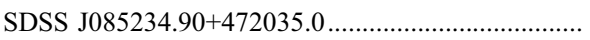 & 21.99 & 0.15 & 18.90 & 0.05 \\
\hline 2MASS J090837.97+503208.0 & 20.06 & 0.03 & 17.22 & 0.02 \\
\hline SDSS J093109.56+032732.5 & 22.00 & 0.15 & 19.28 & 0.05 \\
\hline SDSS J100401.41+005354.9 .... & 21.81 & 0.18 & 19.76 & 0.11 \\
\hline SDSS J103026.78+021306.4 .... & 23.43 & 0.61 & 19.94 & 0.11 \\
\hline SDSS J104409.43+042937.6 & 21.67 & 0.08 & 18.73 & 0.03 \\
\hline 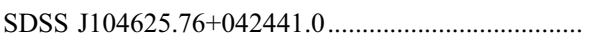 & 22.38 & 0.16 & 19.74 & 0.07 \\
\hline 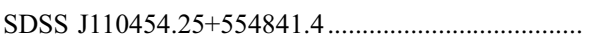 & 22.95 & 0.34 & 19.94 & 0.09 \\
\hline 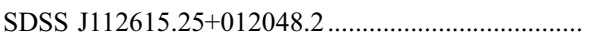 & 22.28 & 0.25 & 19.79 & 0.12 \\
\hline 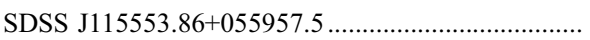 & 21.26 & 0.12 & 18.45 & 0.04 \\
\hline SDSS J115700.50+061105.2 ........ & 24.12 & 0.67 & 20.20 & 0.11 \\
\hline SDSS J120747.17+024424. ${ }^{\mathrm{a}} .$. & 21.47 & 0.11 & 18.41 & 0.04 \\
\hline 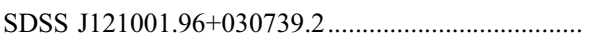 & 22.25 & 0.26 & 19.79 & 0.10 \\
\hline 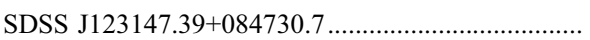 & 22.79 & 0.30 & 18.94 & 0.04 \\
\hline SDSS J133148.90-011651.4 $\ldots$ & 20.56 & 0.08 & 18.14 & 0.04 \\
\hline 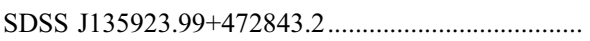 & 23.15 & 0.37 & 19.76 & 0.09 \\
\hline SDSS J140814.74+053952.9 ${ }^{\mathrm{a}} \ldots$ & 20.48 & 0.05 & 18.71 & 0.05 \\
\hline SDSS J143211.74-005900.8 ………………….... & 22.00 & 0.23 & 19.62 & 0.10 \\
\hline SDSS J143535.70-004347. $0^{\mathrm{a}} \ldots \ldots$. & 20.86 & 0.06 & 19.02 & 0.04 \\
\hline 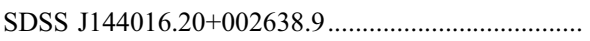 & 20.65 & 0.05 & 18.75 & 0.03 \\
\hline 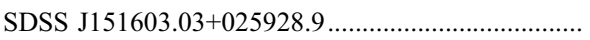 & 22.25 & 0.26 & 19.89 & 0.12 \\
\hline 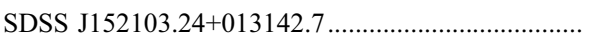 & 24.46 & 0.57 & 19.57 & 0.06 \\
\hline 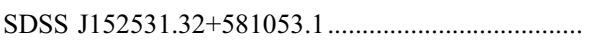 & 22.05 & 0.16 & 19.75 & 0.09 \\
\hline 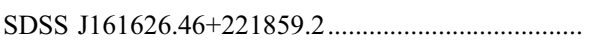 & 23.07 & 0.26 & 20.33 & 0.10 \\
\hline 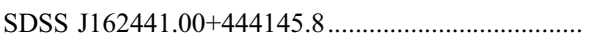 & 23.17 & 0.40 & 20.07 & 0.11 \\
\hline 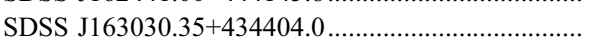 & 22.10 & 0.14 & 19.45 & 0.05 \\
\hline 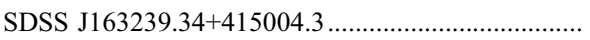 & 23.29 & 0.37 & 20.35 & 0.11 \\
\hline 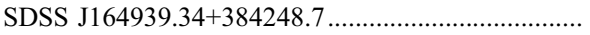 & 22.36 & 0.31 & 20.05 & 0.10 \\
\hline 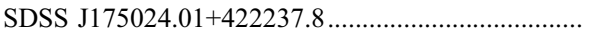 & 24.07 & 0.96 & 19.38 & 0.09 \\
\hline SDSS J175805.46+463311.9 ... & 24.18 & 0.57 & 19.67 & 0.07 \\
\hline SDSS J204749.61-071818.3.. & 23.87 & 0.78 & 19.74 & 0.10 \\
\hline 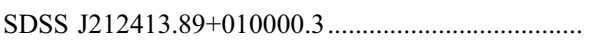 & 23.77 & 0.54 & 19.71 & 0.12 \\
\hline
\end{tabular}

Note. - The SDSS asinh magnitudes (Lupton et al. 1999) corresponding to zero flux are $i=24.4$ and $z=22.8$.

a Optical spectroscopy given by Hawley et al. 2002.

${ }^{b}$ Discovered in the 2MASS database by Cruz et al. 2003.

for objects brighter than about 20 and 19, respectively, while the astrometric accuracy is better than $0{ }^{\prime \prime} 1$ (rms) in each coordinate. The result is a catalog of objects with magnitudes in five bands, positions, and shape parameters (e.g., Abazajian et al. 2003 and references therein).

All $\mathrm{L}$ and T dwarfs are undetected in SDSS $u$ and $g$, and all save the brightest are undetected in $r$. Almost all late $\mathrm{L}$ and $\mathrm{T}$ dwarfs have $i-z>2$; thus, candidate field brown dwarfs are selected from the SDSS photometry to be very red. Given the extremely red colors and low luminosities of these dwarfs, they are often detected only in the $z$ band. Objects this red are rare; at the SDSS magnitude limits they are late L and $\mathrm{T}$ dwarfs, quasars at redshift greater than 5.7 (Fan et al. 2001, 2003 ) or very rare unusual broad absorption line quasars (see Hall et al. 2002), and their surface density is smaller than about one per $50 \mathrm{deg}^{2}$. As a result, $z$-band only detections are 


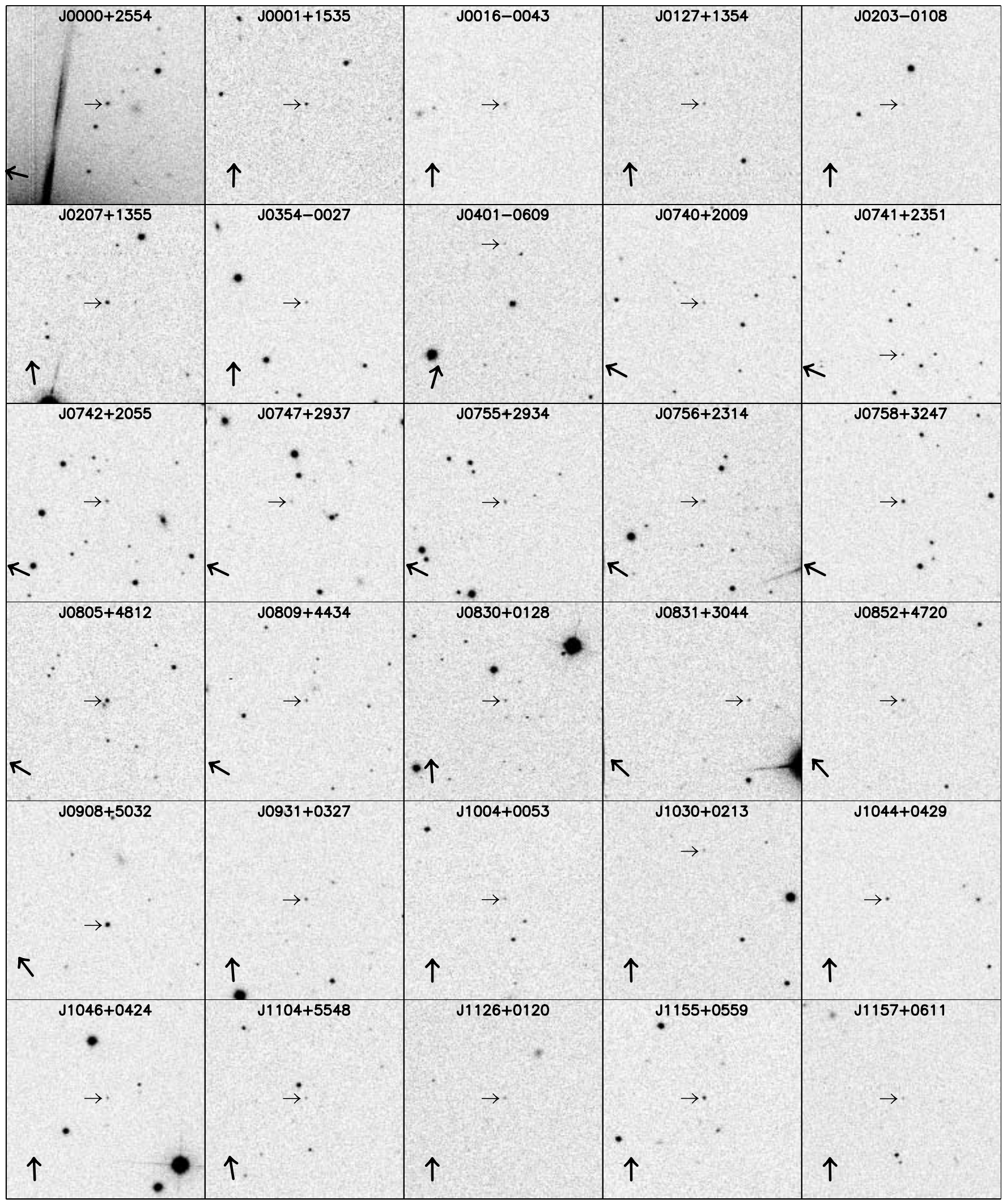

FIG. 1.-SDSS $z$ finding charts of the new dwarfs listed in Table 1. The side of each finding chart is $120^{\prime \prime}$. The large direction arrow points north, with east $90^{\circ}$ counterclockwise from north. The object is indicated by the small arrow. 


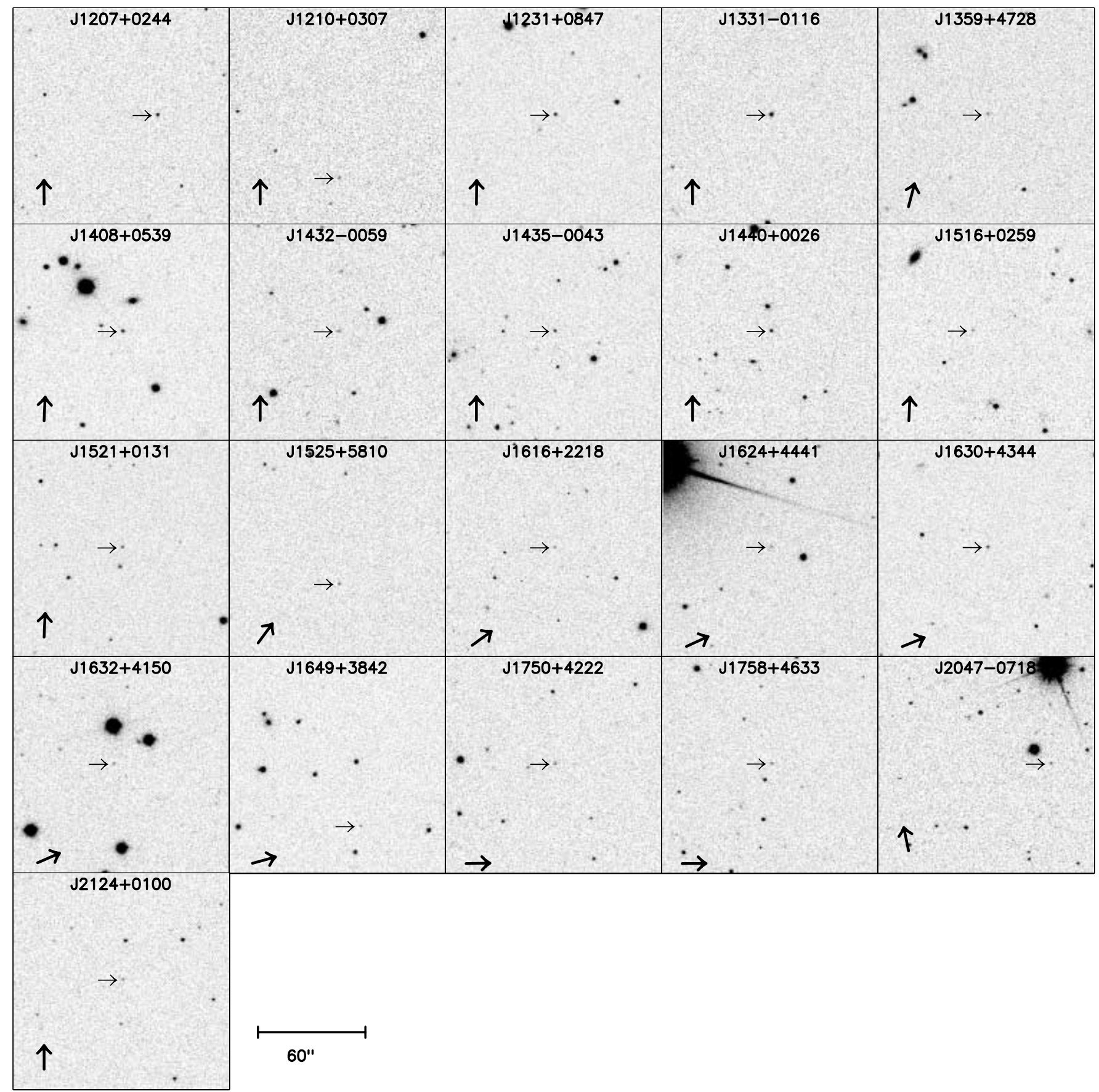

FIG. 1.-Continued

overwhelmed by data artifacts, in particular "cosmic rays" in the $z$ detectors. The winnowing of "objects" to find those that are real is an exhaustive process, consisting of careful analysis of the image to reject cosmic rays (which usually have imprints smaller than the point-spread function), reobservation with another telescope at $z$ band (usually the ARC $3.5 \mathrm{~m}$ telescope at Apache Point Observatory), and comparison with $J$-band observations from 2 MASS or elsewhere. $J$-band photometry also allows a first judgment as to whether an object is a brown dwarf or a high-redshift quasar. These procedures are described in detail by Fan et al. (2001, 2003). The total area searched to date for candidate very red dwarfs in SDSS, including objects in our previous papers and in Table 1, is $2870 \mathrm{deg}^{2}$, so the surface density of T dwarfs found by SDSS is approximately one per $100 \mathrm{deg}^{2}$. The regions of the sky searched are shown by Fan et al. (2003).

Some of the objects in the final sample presented in $\S 4$ have been found to be close binaries, usually from HST imaging. Such objects will be designated by "AB" attached to the names, since the photometric and spectroscopic observations measure the total flux of both members. These known binaries are DENIS-P J0205-1159AB (Koerner et al. 1999; Leggett et al. 2001; Bouy et al. 2003); DENIS-P J1228-1547AB (Koerner et al. 1999; Martín et al. 1999a; Bouy et al. 2003); 2MASS J0746+2000AB and 2MASS J0850+1057AB (Reid et al. 2001; Bouy et al. 2003); 2MASS J1225-2739AB and 2MASS J1534-2952AB (Burgasser et al. 2003d); and 2MASS J1553+1532AB (A. Burgasser 2003, private 
TABLE 2

New UFTI $Z$ PHOTOMETRY

\begin{tabular}{|c|c|c|}
\hline Name & $\begin{array}{c}Z \pm 0.05 \\
(\mathrm{mag})\end{array}$ & $\begin{array}{c}\text { Date } \\
\text { (YYYYMMDD) }\end{array}$ \\
\hline 2MASS J0030-1450... & 18.13 & 20021106 \\
\hline 2MASS J0243-2453... & 16.98 & 20021106 \\
\hline 2MASS J0415-0935 .............. & 17.30 & 20021106 \\
\hline 2MASS J0727+1710 & 17.17 & 20021106 \\
\hline 2MASS J0825+2115 ............ & 16.62 & 20021106 \\
\hline 2MASS J0937+2931_.......... & 16.01 & 20021106 \\
\hline 2MASS J2356-1553 ............... & 17.60 & 20021022 \\
\hline
\end{tabular}

communication). Not all of the objects in the sample have been imaged at high angular resolution, however, and there are likely to be more binaries among them.

\section{NEW OBSERVATIONAL DATA}

\subsection{Near-Infrared Photometry}

Table 2 gives new $Z$ photometry and Table 3 new JHK photometry. The central wavelengths of the filter passbands are $0.95,1.25,1.64$, and $2.2 \mu \mathrm{m}$; more details of the filters and calibration are given by Leggett et al. (2002a). All data were obtained on UKIRT. All Z-band data were obtained with UKIRT's FastTrack Imager (UFTI, Roche et al. 2003) on the dates shown in Table 2. The JHK data were taken with the Mauna Kea consortium filter set (MKO-NIR) on the dates and with the cameras as listed in Table 3. Three cameras were used for the JHK observations - the Infrared Camera (IRCAM, McLean et al. 1986), UFTI, and the UKIRT Imager-Spectrometer (UIST, Ramsay-Howat et al. 2000). UFTI contains a HAWAII $1024 \times$ $1024 \mathrm{HgCdTe}$ detector and has a plate scale of 0.091 pixel $^{-1}$. IRCAM contains an SBRC $256 \times 256 \mathrm{InSb}$ detector and has a plate scale of 0.081 pixel $^{-1}$. UIST contains an ALADDIN $1024 \times 1024 \mathrm{InSb}$ detector and has a choice of plate scales, either $0^{\prime \prime} 061$ or $0^{\prime \prime} 120 \mathrm{pixel}^{-1}$. The $0^{\prime \prime} 120 \mathrm{pixel}^{-1}$ plate scale was used with UIST for these JHK observations. Readout of the full IRCAM array was employed, but $512 \times 512$ subarrays were used with UFTI and UIST to reduce overheads (i.e., to increase efficiency). The fields of view were thus $200^{\prime \prime} 7,466^{\prime \prime} 6$, and 61 ".4 for IRCAM, UFTI, and UIST, respectively.

Individual exposure times were usually $250 \mathrm{~s}$ at $Z$ and $60 \mathrm{~s}$ at each of JHK. Observations were made in a three- or fiveposition dither pattern at $Z$ and with five or nine dither positions at $J H K$. The $J H K$ data were calibrated using the UKIRT Faint Standards of Hawarden et al. (2001), ${ }^{12}$ translated onto the MKO-NIR system using as yet unpublished observations carried out at UKIRT as part of an observatory project to provide calibrators in the MKO-NIR system. The $Z$ data were calibrated using unpublished UKIRT observations. These calibration data are currently available via the UKIRT Web site. ${ }^{13}$

We have investigated the effects of the different optical elements, their coatings, and the detector antireflection coatings on the $J H K$ photometric systems of the three cameras. Synthesizing $J H K$ for $\mathrm{L}$ and $\mathrm{T}$ dwarfs using flux-calibrated spectra (see G02) shows that the differences at $J$ are about 0.009 mag for $\mathrm{L}$ to early $\mathrm{T}$ types and $0.013 \mathrm{mag}$ for late $\mathrm{T}$ dwarfs. At $H$ the differences are around 0.001 mag for objects

\footnotetext{
${ }^{12}$ See also http://www.jach.hawaii.edu/JACpublic/UKIRT/astronomy/calib /fs_newJHK.html.

${ }^{13}$ At http://www.jach.hawaii.edu/JACpublic/UKIRT/astronomy/calib/ fs_izjhklm.dat.
}

of both $\mathrm{L}$ and $\mathrm{T}$ spectral type. At $K$, the difference is $0.001 \mathrm{mag}$ for objects of type L, 0.003 mag for objects of type early T, and $0.010 \mathrm{mag}$ for late $\mathrm{T}$ dwarfs. In all cases this is significantly less than the measurement error, so that the data from the three cameras are effectively on the same photometric system, defined by the MKO-NIR filter set. Transformations between this filter set and other widely used $J H K$ filter sets (e.g., the 2MASS system) are described by Stephens \& Leggett (2004).

\subsection{Spectroscopy and Spectral Types}

Table 4 lists the instrument configurations for the new spectroscopic observations. All spectra were obtained at UKIRT using either the Cooled Grating Spectrometer (CGS4, Wright et al. 1993) or UIST. CGS4 has an SBRC $256 \times 256$ InSb detector with 0 "' 6 pixels. In UIST's spectroscopy mode the ALADDIN array has 0 ". 12 pixels. Individual exposure times were typically $120 \mathrm{~s}$ for the CGS4-Z and CGS4-J settings, $60 \mathrm{~s}$ for CGS4-H and CGS4-K and $120 \mathrm{~s}$ for UIST-HK. The targets were nodded $7^{\prime \prime}-12^{\prime \prime}$ along the slit. A- or earlyto-mid F-type bright stars were used as calibrators to remove the effects of the terrestrial atmosphere, with $\mathrm{H}$ I recombination lines in their spectra removed artificially prior to ratioing. Both instruments have lamps that provide accurate flat fielding and wavelength calibration.

A $\log$ of the measured spectra is given in Table 5 . We concentrated on obtaining spectra in the $H$ and $K$ bands, because indices in these wavelength regions can be used for a wide range of types (G02). Table 6 gives the derived spectral indices and the mean implied type on the G02 scheme; the individual classifications are rounded off to the nearest 0.5 of a subclass, but the mean type is derived from the unrounded values. Errors are given for those dwarfs with multiple indices which show a scatter larger than the estimated classification uncertainty of 0.5 subclasses. The reader is referred to $\mathrm{G} 02$ for examples of the spectral sequences and line identifications. Spectra and photometry from this and our previous papers are available on request or from our $\mathrm{L}$ and $\mathrm{T}$ dwarf Web site. ${ }^{14}$ Note that, unlike G02, we do not incorporate red "PC3" and "Color-d" spectral indices for the current sample. These indices can be used for classifying dwarfs of spectral type L6 and earlier (G02); however, in this paper we present near-infrared data only. A future paper will examine red spectra, where available for the sample, and investigate the wavelengthdependent effects of cloud condensation (see discussion in $\S 5.2$ ).

Table 6 contains 14 new $\mathrm{T}$ dwarfs, including one SDSS object optically classified as L8 by Hawley et al. (2002). The total number of $\mathrm{T}$ dwarfs presently known is 58 , four of which are close binaries. The distribution of new SDSS $\mathrm{T}$ dwarfs is two T0's, three T1-T1.5's, two T2's, two T4.5-T5's, two T5.5's, two T6's, and one T7. The last of these, SDSS $\mathrm{J} 1758+4633$, is the latest-type dwarf found to date in the SDSS. Nine L8-L9.5 dwarfs have been identified from new infrared spectra. Together with the seven new early $\mathrm{T}$ dwarfs, they significantly increase the number of known dwarfs in the $\mathrm{L}-\mathrm{T}$ transition region. Finally, the near-infrared photometry for SDSS J1649+3842 and the photometry and spectroscopy for SDSS J0747+2937 show that they are M dwarfs. The $\mathrm{CH}_{4}-\mathrm{K}$ index for the latter (see Table 6) is at the limit of the G02 scheme and is very uncertain. These $M$ dwarfs are not discussed further. We have not obtained infrared spectra for six of the 51 objects listed in Table 1.

\footnotetext{
${ }^{14}$ At http://www.jach.hawaii.edu/ skl/LTdata.html.
} 
TABLE 3

New MKO-NIR JHK РноTometry

\begin{tabular}{|c|c|c|c|c|c|c|c|c|}
\hline Name & $J$ & $\sigma_{J}$ & $H$ & $\sigma_{H}$ & $K$ & $\sigma_{K}$ & $\begin{array}{c}\text { Date } \\
\text { (YYYYMMDD) }\end{array}$ & Camera \\
\hline 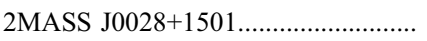 & 16.45 & 0.03 & 15.42 & 0.03 & 14.51 & 0.03 & 20011124 & IRCAM \\
\hline 2MASS J0028+1501............................... & 16.44 & 0.03 & 15.44 & 0.03 & 14.53 & 0.03 & 20020108 & UFTI \\
\hline 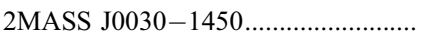 & 16.39 & 0.03 & 15.37 & 0.03 & 14.49 & 0.03 & 20011124 & IRCAM \\
\hline 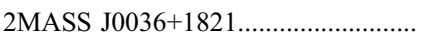 & 12.29 & 0.03 & 11.65 & 0.03 & 11.05 & 0.03 & 20021207 & UIST \\
\hline 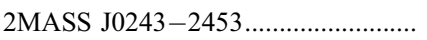 & 15.13 & 0.03 & 15.39 & 0.03 & 15.34 & 0.03 & 20011124 & IRCAM \\
\hline 2MASS J0415-0935 & 15.32 & 0.03 & 15.70 & 0.03 & 15.83 & 0.03 & 20010829 & UFTI \\
\hline 2MASS J0727+1710 & 15.19 & 0.03 & 15.67 & 0.03 & 15.69 & 0.03 & 20011125 & IRCAM \\
\hline 2MASS J0755+2212 & 15.46 & 0.03 & 15.70 & 0.03 & 15.86 & 0.03 & 20020109 & UFTI \\
\hline 2MASS J0801+4628.............................. & 16.21 & 0.03 & 15.31 & 0.03 & 14.58 & 0.03 & 20020109 & UFTI \\
\hline 2MASS J0908+5032 & 14.40 & 0.03 & 13.54 & 0.03 & 12.89 & 0.03 & 20020108 & UFTI \\
\hline 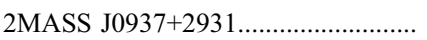 & 14.29 & 0.03 & 14.67 & 0.03 & 15.39 & 0.06 & 20011124 & IRCAM \\
\hline 2MASS J1503+2525 & 13.55 & 0.03 & 13.90 & 0.03 & 13.99 & 0.03 & 20030104 & UIST \\
\hline 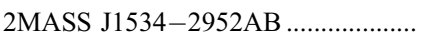 & 14.60 & 0.03 & 14.74 & 0.03 & 14.91 & 0.03 & 20020715 & IRCAM \\
\hline 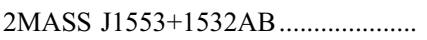 & 15.34 & 0.03 & 15.76 & 0.03 & 15.95 & 0.03 & 20020109 & UFTI \\
\hline 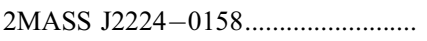 & 13.89 & 0.03 & 12.84 & 0.03 & 11.98 & 0.03 & 20020620 & UFTI \\
\hline 2MASS J2244+2043 & 16.33 & 0.03 & 15.06 & 0.03 & 13.90 & 0.03 & 20020620 & UFTI \\
\hline 2MASS J2254+3123 & 15.01 & 0.03 & 14.95 & 0.03 & 15.03 & 0.03 & 20010829 & UFTI \\
\hline 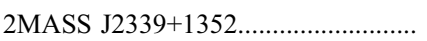 & 15.81 & 0.03 & 16.00 & 0.03 & 16.17 & 0.03 & 20010829 & UFTI \\
\hline 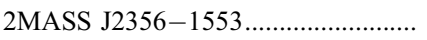 & 15.48 & 0.03 & 15.70 & 0.03 & 15.73 & 0.03 & 20011124 & IRCAM \\
\hline SDSS J0000+2554 & 14.73 & 0.05 & 14.74 & 0.03 & 14.82 & 0.03 & 20031207 & UFTI \\
\hline 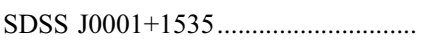 & 15.29 & 0.03 & 14.40 & 0.03 & 13.52 & 0.05 & 20031207 & UFTI \\
\hline 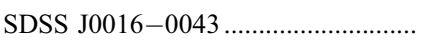 & 16.34 & 0.05 & 15.34 & 0.05 & 14.52 & 0.03 & 20031207 & UFTI \\
\hline 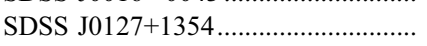 & 16.71 & 0.03 & 15.84 & 0.03 & 15.09 & 0.03 & 20020108 & UFTI \\
\hline SDSS J0203-0108 & 17.83 & 0.05 & 16.87 & 0.03 & 16.18 & 0.03 & 20030104 & UIST \\
\hline SDSS J0207+1355 & 15.27 & 0.03 & 14.45 & 0.03 & 13.81 & 0.03 & 20020109 & UFTI \\
\hline 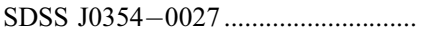 & 17.14 & 0.03 & 16.46 & 0.03 & 15.95 & 0.03 & 20030104 & UIST \\
\hline SDSS J0401-0609 & 17.38 & 0.03 & 16.39 & 0.03 & 15.71 & 0.03 & 20030129 & UFTI \\
\hline 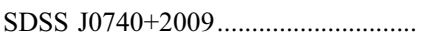 & 16.67 & 0.03 & 15.82 & 0.03 & 15.11 & 0.03 & 20030104 & UIST \\
\hline 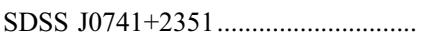 & 15.87 & 0.03 & 16.12 & 0.05 & 16.12 & 0.05 & 20020217 & UFTI \\
\hline 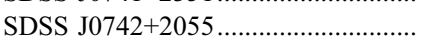 & 15.60 & 0.03 & 15.95 & 0.03 & 16.06 & 0.03 & 20030104 & UIST \\
\hline SDSS J0747+2937 & 17.87 & 0.05 & 17.28 & 0.05 & 16.93 & 0.05 & 20020108 & UFTI \\
\hline SDSS J0755+2934 & 16.71 & 0.03 & 15.94 & 0.03 & 15.32 & 0.03 & 20020109 & UFTI \\
\hline 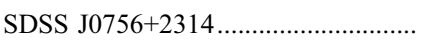 & 16.80 & 0.03 & 15.82 & 0.03 & 15.00 & 0.03 & 20030104 & UIST \\
\hline SDSS J0758+3247 & 14.78 & 0.03 & 14.21 & 0.03 & 13.87 & 0.03 & 20020217 & UFTI \\
\hline SDSS J0805+4812 & 14.61 & 0.03 & 14.01 & 0.03 & 13.51 & 0.03 & 20020109 & UFTI \\
\hline SDSS J0809+4434 & 16.37 & 0.03 & 15.25 & 0.03 & 14.31 & 0.03 & 20020109 & UFTI \\
\hline SDSS J0830+0128 & 15.99 & 0.03 & 16.17 & 0.03 & 16.38 & 0.05 & 20020108 & UFTI \\
\hline SDSS J0831+3044 & 17.45 & 0.05 & 16.89 & 0.05 & 16.35 & 0.05 & 20030105 & UIST \\
\hline SDSS J0852+4720 & 16.13 & 0.03 & 15.21 & 0.03 & 14.62 & 0.03 & 20020108 & UFTI \\
\hline 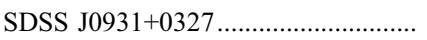 & 16.60 & 0.05 & 16.09 & 0.05 & 15.53 & 0.05 & 20020217 & UFTI \\
\hline 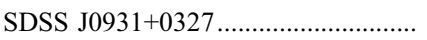 & 16.62 & 0.03 & 16.11 & 0.03 & 15.63 & 0.03 & 20030104 & UIST \\
\hline 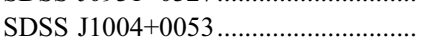 & 17.40 & 0.05 & 16.82 & 0.05 & 16.24 & 0.05 & 20020620 & UFTI \\
\hline SDSS J1030+0213 & 17.10 & 0.05 & 16.27 & 0.05 & 15.67 & 0.05 & 20020108 & UFTI \\
\hline 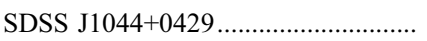 & 15.84 & 0.03 & 14.97 & 0.03 & 14.32 & 0.03 & 20020109 & UFTI \\
\hline 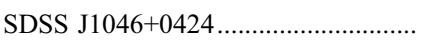 & 16.97 & 0.03 & 16.03 & 0.03 & 15.35 & 0.03 & 20020620 & UFTI \\
\hline 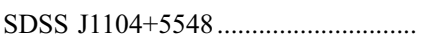 & 17.31 & 0.05 & 16.71 & 0.05 & 16.31 & 0.05 & 20020109 & UFTI \\
\hline 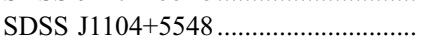 & 17.26 & 0.05 & 16.75 & 0.03 & 16.38 & 0.09 & 20021204 & UIST \\
\hline SDSS J1126+0120 & 16.68 & 0.03 & 15.81 & 0.03 & 15.04 & 0.03 & 20020620 & UFTI \\
\hline SDSS J1155+0559 & 15.63 & 0.03 & 14.74 & 0.03 & 14.09 & 0.03 & 20020109 & UFTI \\
\hline SDSS J1157+0611 & 17.09 & 0.05 & 16.45 & 0.05 & 16.00 & 0.05 & 20010530 & UFTI \\
\hline SDSS J1207+0244 & 15.38 & 0.03 & 14.63 & 0.03 & 14.16 & 0.03 & 20020108 & UFTI \\
\hline SDSS J1210+0307 & 17.27 & 0.05 & 16.58 & 0.03 & 16.03 & 0.03 & 20020620 & UFTI \\
\hline 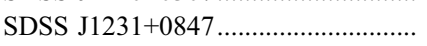 & 15.14 & 0.03 & 15.40 & 0.03 & 15.46 & 0.03 & 20020620 & UFTI \\
\hline 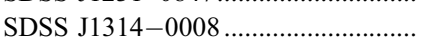 & 16.54 & 0.03 & 15.86 & 0.03 & 15.32 & 0.03 & 20020109 & UFTI \\
\hline 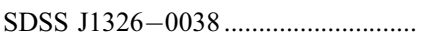 & 16.22 & 0.03 & 15.11 & 0.03 & 14.16 & 0.03 & 20030129 & UFTI \\
\hline SDSS J1331-0116 & 15.34 & 0.03 & 14.67 & 0.03 & 14.09 & 0.03 & 20020109 & UFTI \\
\hline SDSS J1331-0116 & 15.30 & 0.03 & 14.63 & 0.03 & 14.04 & 0.03 & 20020620 & UFTI \\
\hline 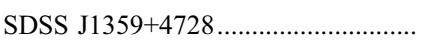 & 16.95 & 0.03 & 16.00 & 0.03 & 15.34 & 0.03 & 20020620 & UFTI \\
\hline SDSS J1408+0539 & 16.49 & 0.03 & 15.95 & 0.03 & 15.49 & 0.03 & 20030104 & UIST \\
\hline 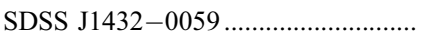 & 16.99 & 0.05 & 16.14 & 0.05 & 15.45 & 0.05 & 20010530 & UFTI \\
\hline 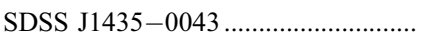 & 16.41 & 0.03 & 15.68 & 0.03 & 15.12 & 0.03 & 20020620 & UFTI \\
\hline SDSS J1440+0026 & 15.93 & 0.03 & 15.23 & 0.03 & 14.64 & 0.03 & 20020620 & UFTI \\
\hline SDSS J1516+0259 & 16.88 & 0.05 & 16.07 & 0.05 & 15.35 & 0.05 & 20010530 & UFTI \\
\hline 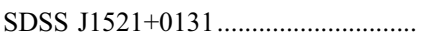 & 16.06 & 0.03 & 15.63 & 0.03 & 15.48 & 0.03 & 20020620 & UFTI \\
\hline
\end{tabular}


TABLE 3-Continued

\begin{tabular}{|c|c|c|c|c|c|c|c|c|}
\hline Name & $J$ & $\sigma_{J}$ & $H$ & $\sigma_{H}$ & $K$ & $\sigma_{K}$ & $\begin{array}{c}\text { Date } \\
\text { (YYYYMMDD) }\end{array}$ & Camera \\
\hline SDSS J1525+5810 & 16.90 & 0.05 & 16.13 & 0.05 & 15.43 & 0.05 & 20020109 & UFTI \\
\hline SDSS J1616+2218 & 17.53 & 0.03 & 16.50 & 0.03 & 15.65 & 0.03 & 20030618 & UIST \\
\hline 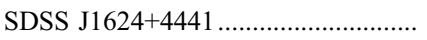 & 17.56 & 0.05 & 16.88 & 0.05 & 16.46 & 0.05 & 20010720 & UFTI \\
\hline SDSS J1630+4344 & 16.48 & 0.03 & 15.51 & 0.03 & 14.70 & 0.03 & 20020620 & UFTI \\
\hline SDSS J1632+4150 & 16.87 & 0.05 & 16.42 & 0.08 & 16.19 & 0.08 & 20020620 & UFTI \\
\hline 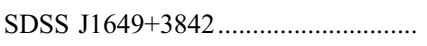 & 17.78 & 0.10 & 17.35 & 0.10 & 16.71 & 0.10 & 20010720 & UFTI \\
\hline 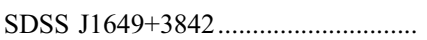 & 17.79 & 0.10 & 17.26 & 0.10 & 16.78 & 0.10 & 20020620 & UFTI \\
\hline 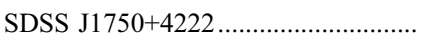 & 16.12 & 0.03 & 15.57 & 0.03 & 15.31 & 0.03 & 20020620 & UFTI \\
\hline 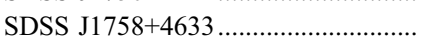 & 15.86 & 0.03 & 16.20 & 0.03 & 16.12 & 0.03 & 20020620 & UFTI \\
\hline SDSS J2047-0718 & 16.70 & 0.03 & 15.88 & 0.03 & 15.34 & 0.03 & 20011125 & IRCAM \\
\hline 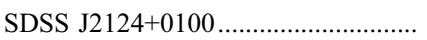 & 15.88 & 0.03 & 16.12 & 0.03 & 16.07 & 0.03 & 20031208 & UFTI \\
\hline 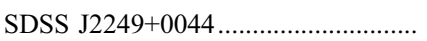 & 16.29 & 0.03 & 15.30 & 0.03 & 14.38 & 0.03 & 20020108 & UFTI \\
\hline
\end{tabular}

The spectrum of 2MASS J0415-0935 has significantly deeper $\mathrm{H}_{2} \mathrm{O}$ and $\mathrm{CH}_{4}$ bands than previously known $\mathrm{T} 8$ dwarfs, and we classify it as T9; it is the latest spectral type dwarf presently known. Figure 2 shows the $H$ - and $K$-band spectra of SDSS J1758+4833 (T7) and 2MASS J0415-0935 (T9), in addition to our previously published spectra of the T6 dwarf SDSS J1624+0029 (Strauss et al. 1999) and the T8 dwarf G1 570D (Geballe et al. 2001). The steady increase in the depths of the $\mathrm{H}_{2} \mathrm{O}$ and $\mathrm{CH}_{4}$ bands from T6 to $\mathrm{T} 9$ can be seen. Figure 2 suggests that there is room for one more T type, which would have essentially zero flux at $1.45,1.7$, and $2.25 \mu \mathrm{m}$. Provisional indices for the end of the T sequence are given in Table 7.

According to the models of Burrows, Sudarsky, \& Lunine (2003), $\mathrm{NH}_{3}$ is expected to be detectable in the $H$ and $K$ spectral regions (at $\sim 1.5,1.95$, and $2.95 \mu \mathrm{m}$ ) for $T_{\text {eff }} \leq 600 \mathrm{~K}$, and its presence may mark the transition to the spectral type after $\mathrm{T}$-although new nonequilibrium chemistry models suggest that the abundance of $\mathrm{NH}_{3}$ may be reduced (Saumon et al. 2003). As we discuss in our companion paper (Golimowski et al. 2004), the effective temperature of 2MASS J0415 - 0935 is $\sim 700 \mathrm{~K}$, too warm for $\mathrm{NH}_{3}$ absorption. There is no sign of $\mathrm{NH}_{3}$ absorption in the spectrum (Fig. 2) or in any other $H K$ spectra we have obtained to date.

\section{THE FINAL SAMPLE}

We have compiled a large sample of $\mathrm{L}$ and $\mathrm{T}$ dwarfs for further study by combining the new data presented in $\S 3$ with our previously published work (Strauss et al. 1999; Fan et al. 2000; Tsvetanov et al. 2000; Leggett et al. 2000, 2001, 2002a, 2002b; Geballe et al. 2001, 2002). This final sample consists of 63 spectroscopically confirmed L dwarfs (59 of which have infrared spectra), six other possible L dwarfs measured photometrically only, and 42 spectroscopically confirmed $\mathrm{T}$ dwarfs.

The distances to 45 of these $\mathrm{L}$ and $\mathrm{T}$ dwarfs are known by virtue of recent parallax measurements or because they are companions to nearby stars with accurately measured parallaxes, either from the ground (van Altena et al. 1995) or from Hipparcos (ESA 1997; Perryman et al. 1997). Since the discovery of isolated $\mathrm{L}$ and $\mathrm{T}$ dwarfs, much effort has been devoted to the measurement of accurate parallaxes, both at optical wavelengths (Tinney et al. 1995; Dahn et al. 2002) and recently at near-infrared wavelengths (Tinney et al. 2003; Vrba et al. 2004). Table 8 presents available parallaxes of $\mathrm{L}$ and $\mathrm{T}$ dwarfs for which we have obtained UKIRT data, together with the derived $K$-band luminosities on the MKO system. Some of the parallaxes are weighted mean values from more than one source, as noted in Table 8 . The errors for $M_{K}$ given in the table are the combined errors in the parallax and in the photometry.

Table 9 summarizes our final sample of $\mathrm{L}$ and $\mathrm{T}$ dwarfs. Column (1) gives full coordinate names for the SDSS and 2MASS dwarfs; these are listed as footnotes for dwarfs discovered in other work (e.g., for Kelu-1; Ruiz et al. 1997). Column (2) lists spectral types from the following sources: this paper (using spectra described in Table 6 or presented by Burgasser et al. 2002a); G02, Kirkpatrick et al. (1999, 2000), and Burgasser et al. (2002a). The uncertainty in the assigned type is given if there are multiple infrared spectral indices that deviate by more than the estimated classification uncertainty of 0.5 subclasses. The spectral type is also flagged if the infrared and optical types (Kirkpatrick et al. 1999, 2000; Hawley et al. 2002; Cruz et al. 2003) differ by more than 1.0 subclass. Discrepant indices, either between the optical and infrared or even within the infrared range, are a sign that the spectra are sampling very different regions of the atmosphere, as we discuss later in $\S \S 5.2$ and 5.7.4. Column (3) lists $M_{J}$, derived from our $J$ measurements and the parallaxes in Table 8. The next two columns list the SDSS $i-z$ and $z$. These values are given only for those objects for which $\sigma_{i}$ or $\sigma_{z}<0.2$ mag. They are based on the most recent SDSS reductions (PHOTO 5425, 2003 July) and may differ slightly

TABLE 4

Spectroscopic Configurations

\begin{tabular}{|c|c|c|c|}
\hline Instrument Configuration & $\begin{array}{l}\text { Wavelength Range } \\
\qquad(\mu \mathrm{m})\end{array}$ & $\begin{array}{l}\text { Resolution } \\
(\AA)\end{array}$ & $\begin{array}{l}\text { Slit Width } \\
\text { (arcsec) }\end{array}$ \\
\hline CGS4-Z. & $0.84-1.04$ & 25 & 1.20 \\
\hline ........................ & $1.03-1.35$ & 21 & 1.20 \\
\hline 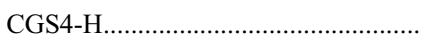 & $1.38-2.02$ & 50 & 1.20 \\
\hline CGS4-K & $1.88-2.52$ & 50 & 1.20 \\
\hline UIST-HK & $1.44-2.49$ & 44 & 0.48 \\
\hline
\end{tabular}


TABLE 5

New Spectroscopy

\begin{tabular}{|c|c|c|c|c|c|}
\hline \multirow[b]{2}{*}{ NAME } & \multicolumn{5}{|c|}{ Date for Observation with Configuration } \\
\hline & $\begin{array}{c}\text { CGS4-Z } \\
\text { (YYYYMMDD) }\end{array}$ & $\begin{array}{c}\text { CGS4-J } \\
\text { (YYYYMMDD) }\end{array}$ & $\begin{array}{c}\text { CGS4-H } \\
\text { (YYYYMMDD) }\end{array}$ & $\begin{array}{c}\text { CGS4-K } \\
\text { (YYYYMMDD) }\end{array}$ & $\begin{array}{c}\text { UIST-HK } \\
\text { (YYYYMMDD) }\end{array}$ \\
\hline 2MASS J0415-0935 .............. & 20021214 & 20021213 & 20020110 & 20020111 & . \\
\hline 2MASS J0727+1710.............. & $\ldots$ & 20021212 & $\ldots$ & $\ldots$ & 20021212 \\
\hline 2MASS J0929+3429.............. & $\ldots$ & $\ldots$ & 20011029 & $\ldots$ & $\ldots$ \\
\hline 2MASS J0908+5032 ................. & 20031208 & 20021212 & 20020110 & 20020111 & $\ldots$ \\
\hline 2MASS J0937+2931.............. & $\ldots$ & 20020112 & 20020110 & 20020111 & $\ldots$ \\
\hline 2MASS J1439+1929............... & $\ldots$ & 20020625 & 20020619 & 20020622 & $\ldots$ \\
\hline 2MASS J1507-1627.............. & $\ldots$ & 20020625 & 20020624 & 20020624 & $\ldots$ \\
\hline 2MASS J2224-0158 ............... & $\ldots$ & 20020714 & 20020618 & 20020622 & $\ldots$ \\
\hline 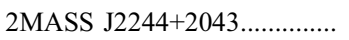 & $\ldots$ & 20021214 & 20020618,21 & $20020618,22,23$ & $\ldots$ \\
\hline 2MASS J2254+3123............... & 20030812 & 20020714 & 20020109 & 20020112 & $\ldots$ \\
\hline 2MASS J2339+1352 ................ & $\ldots$ & 20021214 & 20020625 & 20020625 & $\ldots$ \\
\hline SDSS J0000+2554 ..................... & $\ldots$ & 20031208 & 20031207 & 20031207 & $\ldots$ \\
\hline SDSS J0001+1535 .................... & $\ldots$ & $\ldots$ & $\ldots$ & $\ldots$ & 20031120 \\
\hline SDSS J0016-0043 …................ & $\ldots$ & $\ldots$ & $\ldots$ & $\ldots$ & 20031120 \\
\hline SDSS J0127+1354 .................... & $\ldots$ & $\ldots$ & 20020112 & 20020112 & $\ldots$ \\
\hline SDSS J0203-0108 ................... & $\ldots$ & $\ldots$ & $\ldots$ & $\ldots$ & 20030923 \\
\hline SDSS J0207+1355 ................... & $\ldots$ & $\ldots$ & 20020111 & 20020112 & 20030105 \\
\hline SDSS J0354-0027 ................... & $\ldots$ & $\ldots$ & $\ldots$ & $\ldots$ & 20030105 \\
\hline SDSS J0401-0609 ................... & $\ldots$ & $\ldots$ & $\ldots$ & $\ldots$ & 20030923 \\
\hline SDSS J0741+2351 ................... & $\ldots$ & 20030924 & $\ldots$ & $\ldots$ & 20021130 \\
\hline SDSS J0742+2055 ..................... & $\ldots$ & 20030925 & 20030228 & 20030228 & $\ldots$ \\
\hline SDSS J0747+2937 .................... & $\ldots$ & $\ldots$ & $\ldots$ & $\ldots$ & 20021214 \\
\hline SDSS J0755+2934 ................... & $\ldots$ & $\ldots$ & 20020110 & 20020111 & 20021212 \\
\hline SDSS J0758+3247 ..................... & 20031208 & 20020225 & 20020225 & 20020225 & $\ldots$ \\
\hline 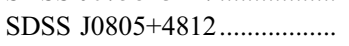 & 20031207 & 20031207 & $\ldots$ & $\ldots$ & 20030321 \\
\hline SDSS J0830+0128 .................... & $\ldots$ & $\ldots$ & 20020110 & $\ldots$ & $\ldots$ \\
\hline SDSS J0852+4720 ..................... & $\ldots$ & 20031124 & 20020110 & 20020111 & $\ldots$ \\
\hline SDSS J0931+0327 .................... & $\ldots$ & 20031207 & $\ldots$ & $\ldots$ & 20021212 \\
\hline SDSS J1004+0053 .................... & $\ldots$ & $\ldots$ & $\ldots$ & $\ldots$ & 20021214 \\
\hline SDSS J1030+0213 ..................... & $\ldots$ & 20031207 & 20020110 & 20020111 & $\ldots$ \\
\hline SDSS J1044+0429 ................... & $\ldots$ & $\ldots$ & 20020111 & 20020111 & $\ldots$ \\
\hline SDSS J1046+0424 ..................... & $\ldots$ & $\ldots$ & $\ldots$ & 20020624 & $\ldots$ \\
\hline SDSS J1104+5548 ...................... & $\ldots$ & $\ldots$ & $\ldots$ & $\ldots$ & 20021213 \\
\hline 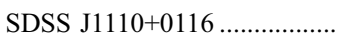 & $\ldots$ & $\ldots$ & $\ldots$ & 20020624 & $\ldots$ \\
\hline SDSS J1126+0120 …................ & $\ldots$ & $\ldots$ & $\ldots$ & 20020622 & $\ldots$ \\
\hline SDSS J1155+0559 ...................... & $\ldots$ & $\ldots$ & 20020111 & 20020112 & $\ldots$ \\
\hline SDSS J1157+0611 …................ & $\ldots$ & 20031208 & 20020110 & 20020112 & $\ldots$ \\
\hline SDSS J1207+0244 „................... & 20040110 & 20020714 & 20020112 & 20020112 & $\ldots$ \\
\hline SDSS J1210+0307 ..................... & $\ldots$ & $\ldots$ & $\ldots$ & $\ldots$ & 20021213 \\
\hline SDSS J1231+0847 .................... & $\ldots$ & 20020625 & 20020619 & 20020622 & $\ldots$ \\
\hline SDSS J1314-0008 ................... & $\ldots$ & $\ldots$ & $\ldots$ & 20020623 & $\ldots$ \\
\hline SDSS J1331-0116 ................... & $\ldots$ & 20031207 & 20020112 & 20020622 & 20021213 \\
\hline SDSS J1359+4728 .................... & $\ldots$ & $\ldots$ & 20020625 & 20020623 & $\ldots$ \\
\hline 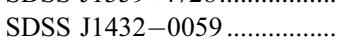 & $\ldots$ & $\ldots$ & 20020621 & 20020622 & $\ldots$ \\
\hline SDSS J1435-0043 …................. & $\ldots$ & $\ldots$ & 20020624 & $\ldots$ & $\ldots$ \\
\hline 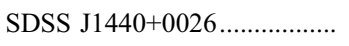 & $\ldots$ & $\ldots$ & 20020624 & $\ldots$ & $\ldots$ \\
\hline SDSS J1516+0259 ...................... & $\ldots$ & $\ldots$ & 20020619 & 20020622 & $\ldots$ \\
\hline 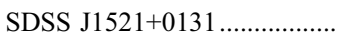 & $\ldots$ & 20030809 & 20020619 & 20020625 & $\ldots$ \\
\hline SDSS J1525+5810 ...................... & $\ldots$ & $\ldots$ & 20020621 & 20020623,25 & $\ldots$ \\
\hline SDSS J1616+2218 .................... & $\ldots$ & $\ldots$ & $\ldots$ & $\ldots$ & 20030618 \\
\hline SDSS J1630+4344 _..................... & $\ldots$ & $\ldots$ & 20020624 & 20020622 & $\ldots$ \\
\hline SDSS J1632+4150 .................... & $\ldots$ & 20030821 & 20020624,20020714 & 20020625 & $\ldots$ \\
\hline SDSS J1750+4222 …............... & $\ldots$ & 20030812 & 20020621 & 20020622 & $\ldots$ \\
\hline SDSS J1758+4633 …................. & $\ldots$ & 20030812 & 20020618 & 20020622,23 & $\ldots$ \\
\hline 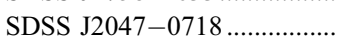 & $\ldots$ & 20030814 & 20020718 & 20020807 & $\ldots$ \\
\hline 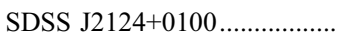 & $\ldots$ & $\ldots$ & 20031208 & $\ldots$ & $\ldots$ \\
\hline
\end{tabular}


TABLE 6

Spectral Indices

\begin{tabular}{|c|c|c|c|c|c|c|c|c|c|}
\hline \multirow[b]{2}{*}{ Name } & \multicolumn{2}{|c|}{$\mathrm{H}_{2} \mathrm{O}(\mathrm{J})$} & \multicolumn{2}{|c|}{$\mathrm{H}_{2} \mathrm{O}(\mathrm{H})$} & \multicolumn{2}{|c|}{$\mathrm{CH}_{4}(\mathrm{H})$} & \multicolumn{2}{|c|}{$\mathrm{CH}_{4}(\mathrm{~K})$} & \multirow[b]{2}{*}{ MEAn Type } \\
\hline & Index & Type & Index & Type & Index & Type & Index & Type & \\
\hline 2MASS J0415-0935 ......... & 26.35 & T9.5 & 18.66 & T9 & 9.99 & $\mathrm{~T} 8.5$ & 25.26 & T9 & T9 \\
\hline 2MASS J0727+1700.......... & 10.69 & $\mathrm{~T} 7.5$ & 11.69 & $\mathrm{~T} 8.5$ & 6.09 & $\mathrm{~T} 7.5$ & 16.26 & $\mathrm{~T} 8$ & $\mathrm{~T} 8$ \\
\hline 2MASS J0908+5032 .......... & 1.41 & {$[<\mathrm{T} 0]$} & 1.92 & L9.5 & 1.04 & T0 & 1.12 & L7.5 & $\mathrm{L} 9 \pm 1.0$ \\
\hline 2MASS J0929+3429.......... & $\ldots$ & $\ldots$ & 1.72 & L7.5 & 0.96 & {$[<\mathrm{T} 0]$} & $\ldots$ & $\ldots$ & L7.5 \\
\hline 2 MASS J0937+2931 a $\ldots \ldots . .$. & 6.05 & $\mathrm{~T} 6.5$ & 6.85 & T6.5 & 3.70 & T6.5 & 4.39 & $\mathrm{~T} 5$ & T6 \\
\hline 2MASS J1439+1929.......... & 1.10 & {$[<\mathrm{T} 0]$} & 1.30 & $\mathrm{~L} 1$ & 0.96 & {$[<\mathrm{T} 0]$} & 0.95 & {$[<\mathrm{L} 3]$} & $\mathrm{L} 1$ \\
\hline 2MASS J1507-1627......... & 1.31 & {$[<\mathrm{T} 0]$} & 1.59 & L5.5 & 0.98 & {$[<\mathrm{T} 0]$} & 1.03 & L5.5 & L5.5 \\
\hline 2MASS J2224-0158 ......... & 1.37 & {$[<\mathrm{T} 0]$} & 1.48 & L3 & 0.97 & {$[<\mathrm{T} 0]$} & 0.95 & L4 & L3.5 \\
\hline 2MASS J2244+2043 .......... & 1.62 & T0 & 1.71 & L7.5 & 0.91 & {$[<\mathrm{T} 0]$} & 0.99 & L4.5 & $\mathrm{L} 7.5 \pm 2.0$ \\
\hline 2MASS J2254+3123........... & 2.46 & $\mathrm{~T} 3.5$ & 3.69 & $\mathrm{~T} 3.5$ & 1.83 & $\mathrm{~T} 4.5$ & 3.36 & $\mathrm{~T} 4$ & $\mathrm{~T} 4$ \\
\hline 2MASS J2339+1352.......... & 4.08 & $\mathrm{~T} 5$ & 7.26 & T6.5 & 2.35 & $\mathrm{~T} 5.5$ & 5.03 & T5 & $\mathrm{T} 5.5$ \\
\hline SDSS J0000+2554 ................ & 3.03 & $\mathrm{~T} 4.5$ & 4.11 & $\mathrm{~T} 4$ & 1.84 & $\mathrm{~T} 4.5$ & 3.70 & $\mathrm{~T} 4.5$ & $\mathrm{~T} 4.5$ \\
\hline SDSS J0001+1535 ............... & $\ldots$ & $\ldots$ & 1.59 & L5.5 & 0.95 & {$[<\mathrm{T} 0]$} & 0.92 & L3 & $\mathrm{L} 4 \pm 1.0$ \\
\hline SDSS J0016-0043 ............... & $\ldots$ & $\ldots$ & 1.61 & L5.5 & 0.85 & {$[<\mathrm{T} 0]$} & 1.03 & L5.5 & L5.5 \\
\hline SDSS J0127+1354 .............. & $\ldots$ & $\ldots$ & 1.58 & L5 & 0.96 & {$[<\mathrm{T} 0]$} & 1.09 & L7 & $\mathrm{L} 6 \pm 1.0$ \\
\hline SDSS J0203-0108 …......... & $\ldots$ & $\ldots$ & 2.00 & L9.5 & 1.04 & T0 & 1.21 & L9 & L9.5 \\
\hline SDSS J0207+1355 ${ }^{\mathrm{b}} \ldots \ldots \ldots \ldots$ & $\ldots$ & $\ldots$ & 1.37 & $\mathrm{~L} 2$ & 0.98 & {$[<\mathrm{T} 0]$} & 0.97 & L4.5 & $\mathrm{L} 3 \pm 1.5$ \\
\hline SDSS J0354-0027 ............... & $\ldots$ & $\ldots$ & 1.31 & L1 & 1.00 & {$[<\mathrm{T} 0]$} & 0.93 & L3 & $\mathrm{L} 2 \pm 1.0$ \\
\hline SDSS J0401-0609 .............. & $\ldots$ & $\ldots$ & 1.43 & $\mathrm{~L} 2.5$ & 0.98 & {$[<\mathrm{T} 0]$} & 1.01 & L5 & $\mathrm{L} 4 \pm 1.5$ \\
\hline SDSS J0741+2351 ............... & 4.56 & $\mathrm{~T} 5.5$ & 4.65 & $\mathrm{~T} 4.5$ & 2.71 & T5.5 & 5.50 & T5.5 & T5.5 \\
\hline SDSS J0742+2055 ................ & 3.88 & $\mathrm{~T} 5$ & 4.78 & $\mathrm{~T} 5$ & 2.74 & T5.5 & 5.10 & $\mathrm{~T} 5.5$ & $\mathrm{~T} 5$ \\
\hline SDSS J0747+2937 ................ & $\ldots$ & $\ldots$ & 1.07 & M6 & 0.94 & {$[<\mathrm{T} 0]$} & 0.98 & {$[\mathrm{~L} 4.5]$} & late $\mathrm{M}$ \\
\hline SDSS J0755+2934 ${ }^{\mathrm{b}} \ldots \ldots \ldots \ldots$ & $\ldots$ & $\ldots$ & 1.41 & $\mathrm{~L} 2.5$ & 0.99 & {$[<\mathrm{T} 0]$} & 0.97 & L 4.5 & $\mathrm{~L} 3.5 \pm 1.0$ \\
\hline SDSS J0758+3247 ................ & 2.47 & $\mathrm{~T} 3.5$ & 2.67 & $\mathrm{~T} 2$ & 1.12 & $\mathrm{~T} 1$ & 1.59 & $\mathrm{~T} 1.5$ & $\mathrm{~T} 2 \pm 1.0$ \\
\hline SDSS J0805+4812 …........... & 1.38 & {$[<\mathrm{T} 0]$} & 2.01 & L9.5 & 1.06 & $\mathrm{~T} 0.5$ & 1.07 & L6.5 & $\mathrm{L} 9 \pm 1.5$ \\
\hline SDSS J0830+0128 ................ & $\ldots$ & $\ldots$ & 6.25 & $\mathrm{~T} 6$ & 2.44 & $\mathrm{~T} 5.5$ & $\ldots$ & $\ldots$ & T5.5 \\
\hline SDSS J0852+4720 ……....... & 1.44 & {$[<\mathrm{T} 0]$} & 2.14 & $\mathrm{~T} 0.5$ & 1.07 & $\mathrm{~T} 0.5$ & 1.15 & L8 & $\mathrm{L} 9.5 \pm 1.0$ \\
\hline SDSS J0931+0327 .............. & 1.46 & {$[<\mathrm{T} 0]$} & 1.89 & L9 & 0.90 & {$[<\mathrm{T} 0]$} & 1.06 & L6 & $\mathrm{L} 7.5 \pm 1.5$ \\
\hline SDSS J1004+0053 ................ & $\ldots$ & $\ldots$ & 1.29 & $\mathrm{~L} 1$ & 0.92 & {$[<\mathrm{T} 0]$} & 0.92 & L3 & $\mathrm{L} 2 \pm 1.0$ \\
\hline SDSS J1030+0213 ................ & 1.48 & {$[<\mathrm{T} 0]$} & 2.24 & $\mathrm{~T} 1$ & 1.06 & $\mathrm{~T} 0.5$ & 1.14 & L8 & $\mathrm{L} 9.5 \pm 1.0$ \\
\hline SDSS J1044+0429 ................. & $\ldots$ & $\ldots$ & 1.64 & L6.5 & 0.98 & {$[<\mathrm{T} 0]$} & 1.11 & L7 & L7 \\
\hline SDSS J1046+0424 ................ & $\ldots$ & $\ldots$ & $\ldots$ & $\ldots$ & . . & $\ldots$ & 1.06 & L6 & L6 \\
\hline SDSS J1104+5548 ................. & $\ldots$ & $\ldots$ & 1.98 & L9.5 & 1.09 & $\mathrm{~T} 1$ & 1.17 & L7.5 & $\mathrm{L} 9.5 \pm 1.5$ \\
\hline SDSS J1110+0116 …............ & 5.07 & $\mathrm{~T} 6$ & 5.37 & $\mathrm{~T} 5.5$ & 2.47 & $\mathrm{~T} 5.5$ & 6.28 & $\mathrm{~T} 6$ & $\mathrm{~T} 5.5$ \\
\hline SDSS J1126+0120 & $\ldots$ & $\ldots$ & $\ldots$ & $\ldots$ & & $\ldots$ & 1.05 & L6 & L6 \\
\hline SDSS J1155+0559 ............... & $\ldots$ & $\ldots$ & 1.74 & L8 & 0.97 & {$[<\mathrm{T} 0]$} & 1.09 & L7 & L7.5 \\
\hline SDSS J1157+0611 ............... & 1.78 & $\mathrm{~T} 1$ & 3.00 & $\mathrm{~T} 2.5$ & 1.14 & $\mathrm{~T} 1.5$ & 1.45 & $\mathrm{~T} 1$ & $\mathrm{~T} 1.5$ \\
\hline SDSS J1207+0244 ................ & 1.64 & T0 & 2.15 & $\mathrm{~T} 0.5$ & 1.06 & $\mathrm{~T} 0.5$ & 1.24 & L9.5 & T0 \\
\hline SDSS J1210+0307 ............... & $\ldots$ & $\ldots$ & 1.28 & L0.5 & 0.94 & {$[<\mathrm{T} 0]$} & 0.91 & $\mathrm{~L} 2.5$ & $\mathrm{~L} 1.5 \pm 1.0$ \\
\hline SDSS J1231+0847 ............... & 5.54 & T6 & 6.51 & $\mathrm{~T} 6$ & 2.94 & $\mathrm{~T} 6$ & 5.21 & $\mathrm{~T} 5.5$ & T6 \\
\hline SDSS J1314-0008 ............... & $\ldots$ & $\ldots$ & 1.39 & $\mathrm{~L} 2$ & 1.00 & {$[<\mathrm{T} 0]$} & 0.98 & L4.5 & $\mathrm{L} 3.5 \pm 1.5$ \\
\hline SDSS J1331-0116 ................ & 1.40 & {$[<\mathrm{T} 0]$} & $2.12,2.19$ & $\mathrm{~T} 0, \mathrm{~T} 0.5$ & $1.00,0.96$ & {$[<\mathrm{T} 0]$} & $1.03,1.05$ & L5.5,L6 & $\mathrm{L} 8 \pm 2.5$ \\
\hline SDSS J1359+4727 ............... & $\ldots$ & $\ldots$ & 1.77 & L8 & 1.00 & {$[<\mathrm{T} 0]$} & 1.18 & L8.5 & L8.5 \\
\hline SDSS J1432-0059 ................... & $\ldots$ & $\ldots$ & 1.53 & L4 & 0.95 & {$[<\mathrm{T} 0]$} & 1.01 & L5 & L4.5 \\
\hline SDSS J1435-0043 ............... & $\ldots$ & $\ldots$ & 1.43 & $\mathrm{~L} 2.5$ & 0.97 & {$[<\mathrm{T} 0]$} & $\ldots$ & $\ldots$ & $\mathrm{L} 2.5$ \\
\hline SDSS J1440+0026 ............... & $\ldots$ & $\ldots$ & 1.32 & $\mathrm{~L} 1$ & 0.98 & {$[<\mathrm{T} 0]$} & $\ldots$ & $\ldots$ & L1 \\
\hline SDSS J1516+0259 .............. & $\ldots$ & $\ldots$ & 2.11 & T0 & 1.14 & $\mathrm{~T} 1.5$ & 1.16 & L8 & $\mathrm{T} 0 \pm 1.5$ \\
\hline SDSS J1521+0131 ................ & 2.07 & $\mathrm{~T} 2$ & 3.07 & $\mathrm{~T} 2.5$ & 1.10 & $\mathrm{~T} 1$ & 2.01 & $\mathrm{~T} 2.5$ & $\mathrm{~T} 2$ \\
\hline SDSS J1525+5810 ................ & $\ldots$ & $\ldots$ & 1.70 & L7.5 & .. & $\ldots$ & 1.03 & L5.5 & $\mathrm{L} 6.5 \pm 1.0$ \\
\hline SDSS J1616+2218 ................ & $\ldots$ & $\ldots$ & 1.46 & L3 & 0.95 & {$[<\mathrm{T} 0]$} & 1.08 & L6.5 & $\mathrm{L} 5 \pm 2.0$ \\
\hline SDSS J1630+4344 ................ & $\ldots$ & $\ldots$ & 1.76 & L8 & 1.01 & {$[<\mathrm{T} 0]$} & 1.03 & L5.5 & $\mathrm{L} 7 \pm 1.5$ \\
\hline SDSS J1632+4150 ............... & 1.59 & T0 & 2.13 & T0 & 1.15 & $\mathrm{~T} 1.5$ & 1.81 & $\mathrm{~T} 2$ & $\mathrm{~T} 1 \pm 1.0$ \\
\hline SDSS J1750+4222 …............ & 1.75 & $\mathrm{~T} 1$ & 2.21 & $\mathrm{~T} 0.5$ & 1.09 & $\mathrm{~T} 1$ & 1.69 & $\mathrm{~T} 1.5$ & $\mathrm{~T} 1$ \\
\hline SDSS J1758+4633 ................ & 8.63 & $\mathrm{~T} 7$ & 9.08 & $\mathrm{~T} 7.5$ & 4.16 & T6.5 & 10.23 & $\mathrm{~T} 7$ & $\mathrm{~T} 7$ \\
\hline SDSS J2047-0718 ................ & 1.42 & {$[<\mathrm{T} 0]$} & 1.85 & L9 & 1.08 & $\mathrm{~T} 0.5$ & 1.18 & L8.5 & $\mathrm{L} 9.5 \pm 1.0$ \\
\hline SDSS J2124+0100 .................... & $\ldots$ & $\ldots$ & 6.42 & T6 & 2.40 & $\mathrm{~T} 5.5$ & $\ldots$ & $\ldots$ & $\mathrm{T} 6$ \\
\hline
\end{tabular}

${ }^{\text {a }}$ Mean type includes the $K$-band index for this dwarf with suppressed $K$-band flux; see $\S 5$ 5.6.

b Based on UIST data only, more consistent than previous CGS4 data. 


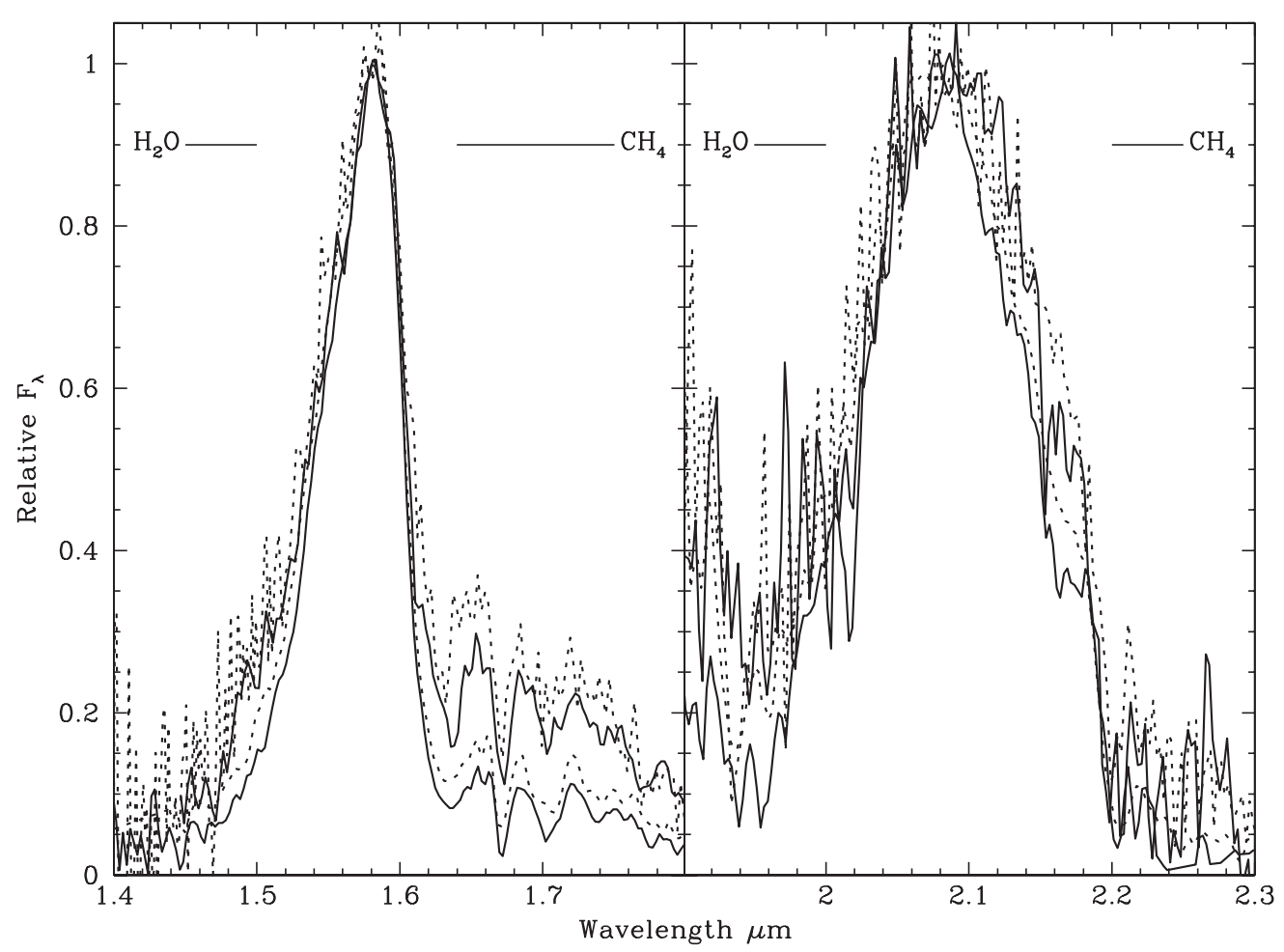

FIG. 2.-Normalized $H$ and $K$ spectra $(R \approx 400)$ for (top to bottom) SDSS J1624+0029 (T6), SDSS J1758+4633 (T7), G1 570D (T8), and 2MASS J0415-0935 (T9). The spectra for SDSS J1624+0029 (Strauss et al. 1999) and Gl 570D (Geballe et al. 2001) are shown as dotted lines.

from previously published values. SDSS photometry for nonSDSS dwarfs, where available, are included in Table 9. The remaining columns list $z-J, Z-J, J, J-K, J-H$, and $H-K$. Some near-infrared magnitudes and colors are synthesized from the spectra, as noted in the table. A small number of dwarfs have detectable SDSS $r$-band fluxes; those dwarfs for which $\sigma_{r}<0.3$ mag are given in Table 10. Note again that the SDSS $r, i$, and $z$ measurements are on the AB system, while the other magnitudes are on the Vega $=0$ system. $Z$ is on the UKIRT UFTI photometric system, while $J, H$, and $K$ are on the MKO-NIR system.

For most of the objects in the sample, we have only single measurements of JHK. However, we have obtained repeat photometry for a few objects with unusual colors. For each of the following dwarfs two measurements were obtained that agree to within the observational uncertainties, and the results are simply averaged in Table 9: 2MASS J0036+1821 (observed on 20001205 and 20021207); SDSS J0107+0041 (19991017, 20010124); SDSS J0830+4828 (20001119, 20001206); SDSS J0931+0327 (20020217, 20030104); SDSS $\mathrm{J} 1104+5548$ (20020109, 20031204), and SDSS J1331-0116 (20020109, 20020620). We have discarded data obtained on 20000314 (Leggett et al. 2002a) for two objects. The $J$ measurements for SDSS J1314-0008 appear to be spuriously bright; we have combined the $H$ and $K$ data from that night with the $J H K$ data presented in Table 3 to produce the colors given in Table 9. All of the $J H K$ data taken on that same night for SDSS J1326-0038 appear to be discrepant, and we have averaged data from 20010124 and 20030129 in Table 9. Finally, data for two of the redder mid L dwarfs (2MASS J0028+1501 and SDSS J2249+0044) suggest that they may be variable at the $5 \%-10 \%$ level, which is not unexpected, given published detections of variability of $\mathrm{L}$ dwarfs and the possibility that nonuniform clouds exist in their atmospheres (e.g., Gelino et al. 2002, Enoch et al. 2003, and the discussion in $\S 5$ 5). Including data taken with IRCAM on 20001119 that were discarded by Leggett et al. (2002a), four measurements exist for 2MASS J0028+1501 and three for SDSS J2249+0044. The former object shows variations at JHK of about $0.05 \mathrm{mag}$, while the latter object varies by about $0.1 \mathrm{mag}$ at each of $J H K$. Weighted means are given for these two objects in Table 9, and the individual data sets are listed in the footnotes.

\section{DISCUSSION}

\subsection{Characteristics of $L$ and $T$ Dwarf Atmospheres}

As effective temperatures of dwarfs cool to those of the late $\mathrm{M}$ dwarfs and below, two chemical changes occur in their photospheres that strongly impact their emergent spectral energy distributions. The first to occur, for late M dwarfs, is

TABLE 7

Provisional Spectral Indices for the End of the T Sequence

\begin{tabular}{|c|c|c|c|c|}
\hline Type & $\mathrm{H}_{2} \mathrm{O} 1.2 \mu \mathrm{m}$ & $\mathrm{H}_{2} \mathrm{O} 1.5 \mu \mathrm{m}$ & $\mathrm{CH}_{4} 1.6 \mu \mathrm{m}$ & $\mathrm{CH}_{4} 2.2 \mu \mathrm{m}$ \\
\hline 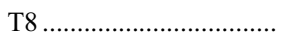 & $10-15$ & $9-12$ & $6-9$ & $12-18$ \\
\hline . & $15-30$ & $12-24$ & $9-18$ & $18-36$ \\
\hline $\mathrm{T} 10 \ldots \ldots \ldots \ldots \ldots$ & $>30$ & $>24$ & $>18$ & $>36$ \\
\hline
\end{tabular}


TABLE 8

Parallaxes and Absolute $K$ Magnitudes

\begin{tabular}{|c|c|c|c|c|c|c|}
\hline Name & $\begin{array}{c}\pi^{\mathrm{a}} \\
(\mathrm{mas})\end{array}$ & $\begin{array}{c}\text { Error } \\
(\pi \text { mas })\end{array}$ & $M_{K}$ & Error $M_{K}$ & Spectral Type & Reference $^{b}$ \\
\hline 2MASS J0030-1450......................... & 37.4 & 4.5 & 12.36 & 0.26 & L7 & V03 \\
\hline 2MASS J0036+1821........................ & 114.2 & 0.8 & 11.33 & 0.03 & L4 & D02 \\
\hline 2MASS J0328+2302... & 33.1 & 4.2 & 12.47 & 0.28 & L9.5 & V03 \\
\hline 2MASS J0345+2540........................... & 37.1 & 0.5 & 10.51 & 0.04 & L1 & D02 \\
\hline 2MASS J0415-0935 ......................... & 174.3 & 2.8 & 17.04 & 0.04 & T9 & V03 \\
\hline 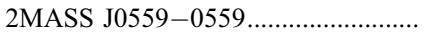 & 96.7 & 1.0 & 13.66 & 0.04 & $\mathrm{~T} 4.5$ & D02,V03 \\
\hline 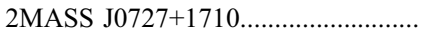 & 110.1 & 2.3 & 15.90 & 0.05 & T8 & V03 \\
\hline 2MASS J0746+2000AB .................... & 81.9 & 0.3 & 10.00 & 0.03 & L1 & D02 \\
\hline 2MASS J0825+2115 ......................... & 94.2 & 0.9 & 12.80 & 0.04 & L6 & $\mathrm{D} 02, \mathrm{~V} 03$ \\
\hline 2MASS J0850+1057AB ...................... & 33.8 & 2.7 & 12.00 & 0.18 & L6 & D02,V03 \\
\hline 2MASS J0937+2931_....................... & 162.8 & 3.9 & 16.45 & 0.08 & T6 & V03 \\
\hline 2MASS J1047+2124_..................... & 98.8 & 3.3 & 16.17 & 0.08 & T6.5 & V03,T03 \\
\hline 2MASS J1217-0311 .......................... & 93.2 & 2.1 & 15.77 & 0.06 & $\mathrm{~T} 8$ & V03,T03 \\
\hline 2MASS J1225-2739AB ................... & 74.8 & 2.0 & 14.65 & 0.07 & T6 & V03,T03 \\
\hline 2MASS J1439+1929.... & 69.6 & 0.5 & 10.68 & 0.03 & L1 & $\mathrm{D} 02$ \\
\hline 2MASS J1507-1627.......................... & 136.4 & 0.6 & 11.96 & 0.03 & L5.5 & D02 \\
\hline 2 MASS J1523+3014 ${ }^{\mathrm{c}} \ldots \ldots \ldots \ldots \ldots \ldots \ldots$ & 54.4 & 1.1 & 13.03 & 0.06 & L8 & Hip,YPC,V03 \\
\hline 2MASS J1534-2952AB .................. & 73.6 & 1.2 & 14.24 & 0.06 & T5.5 & T03 \\
\hline 2MASS J1632+1904_....................... & 65.0 & 1.8 & 13.03 & 0.07 & L7.5 & D02,V03 \\
\hline 2MASS J2224-0158........................ & 87.0 & 0.9 & 11.68 & 0.04 & L3.5 & D02,V03 \\
\hline 2MASS J2356-1553 ......................... & 69.0 & 3.4 & 14.92 & 0.11 & T6 & V03 \\
\hline DENIS-P J0205.4-1159AB .............. & 50.6 & 1.5 & 11.51 & 0.07 & L5.5 & D02 \\
\hline DENIS-P J1058.7-1548 ................... & 57.7 & 1.0 & 11.36 & 0.05 & L3 & D02 \\
\hline DENIS-P J1228.2-15AB ................. & 49.4 & 1.9 & 11.18 & 0.09 & L6 & D02 \\
\hline 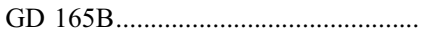 & 31.7 & 2.5 & 11.59 & 0.17 & L3 & YPC \\
\hline 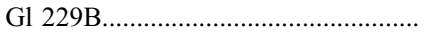 & 173.2 & 1.1 & 15.55 & 0.03 & T6 & Hip,YPC \\
\hline 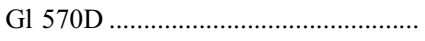 & 170.2 & 1.4 & 16.67 & 0.04 & $\mathrm{~T} 8$ & Hip,YPC \\
\hline 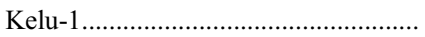 & 53.6 & 2.0 & 10.43 & 0.09 & L3 & D02 \\
\hline 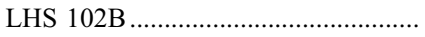 & 104.7 & 11.4 & 11.46 & 0.24 & L4.5 & YPC \\
\hline 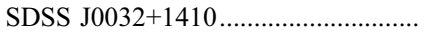 & 30.1 & 5.2 & 12.39 & 0.37 & L8 & V03 \\
\hline 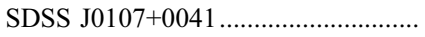 & 64.1 & 4.5 & 12.61 & 0.16 & L5.5 & V03 \\
\hline 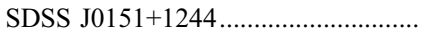 & 46.7 & 3.4 & 13.53 & 0.16 & $\mathrm{~T} 1$ & V03 \\
\hline 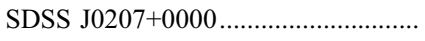 & 34.9 & 9.9 & 14.33 & 0.62 & $\mathrm{~T} 4.5$ & V03 \\
\hline 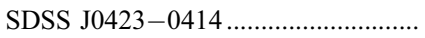 & 65.9 & 1.7 & 12.05 & 0.06 & T0 & V03 \\
\hline 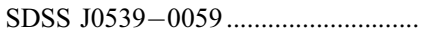 & 76.1 & 2.2 & 11.81 & 0.07 & L5 & V03 \\
\hline 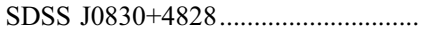 & 76.4 & 3.4 & 13.10 & 0.10 & L9 & V03 \\
\hline SDSS J0837-0000 ........................... & 33.7 & 13.5 & 13.62 & 0.87 & $\mathrm{~T} 0.5$ & V03 \\
\hline 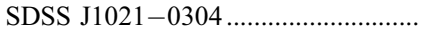 & 35.4 & 4.2 & 13.00 & 0.26 & $\mathrm{~T} 3$ & V03,T03 \\
\hline 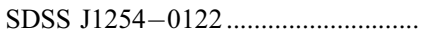 & 74.0 & 1.6 & 13.22 & 0.06 & $\mathrm{~T} 2$ & V03,T03 \\
\hline 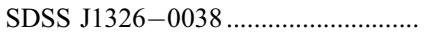 & 50.0 & 6.3 & 12.66 & 0.28 & L5.5 & V03 \\
\hline 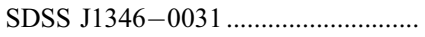 & 69.1 & 2.1 & 14.93 & 0.07 & T6 & V03,T03 \\
\hline 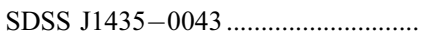 & 16.1 & 5.8 & 11.15 & 0.78 & $\mathrm{~L} 2.5$ & V03 \\
\hline SDSS J1446+0024 ........................... & 45.5 & 3.2 & 12.09 & 0.16 & L5 & V03 \\
\hline 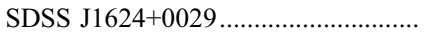 & 90.7 & 1.0 & 15.40 & 0.04 & T6 & $\mathrm{D} 02, \mathrm{~V} 03, \mathrm{~T} 03$ \\
\hline 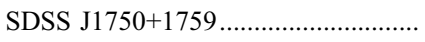 & 36.2 & 4.5 & 13.82 & 0.27 & $\mathrm{~T} 3.5$ & V03 \\
\hline
\end{tabular}

\footnotetext{
a Parallaxes are weighted means of the values given in the cited references (see Golimowski et al. 2004).

b References for parallax: (Hip) Hipparcos Astrometric Catalogue (ESA 1997, Perryman et al. 1997); (YPC) Yale Parallax Catalogue (van Altena et al. 1995); (D02) Dahn et al. 2002; (V03) Vrba et al. 2004; (T03) Tinney et al. 2003.

c 2 MASS J1523+3014=Gl 584C.
}

the appearance of corundum $\left(\mathrm{Al}_{2} \mathrm{O}_{3}\right)$ grains within the photosphere (Jones \& Tsuji 1997) and the formation of condensate clouds. At the even lower effective temperatures of the L dwarfs iron and silicate are the most important condensates. ${ }^{15}$ The effect of the clouds is to weaken or veil the molecular absorption bands and to redden the $J H K$ colors of $\mathrm{L}$ dwarfs

\footnotetext{
15 These condensates are frequently termed "dust," but this can be misleading since in many dwarfs the iron will be in the liquid phase (Lodders 1999). Hence we generally prefer the terms "condensate" to refer to grains or drops of the condensed phase, and "cloud" as the region in the atmosphere within which the condensed species are found.
}

(see, e.g., Ackerman \& Marley 2001; Allard et al. 2001; Marley et al. 2002; Tsuji \& Nakajima 2003). The extent of these effects depends on the number, size, and vertical distribution of the condensates; for spectral modeling these parameters must either be computed from a model or somehow specified. Ackerman \& Marley (2001) developed a onedimensional model of mixing and sedimentation for this purpose. In their model upward vertical mixing of gas and condensate replaces condensates that fall through the cloud base, while far above the cloud base sedimentation efficiently cleanses the atmosphere of condensates. Tsuji \& Nakajima (2003) model the cloud by specifying the temperature range 
TABLE 9

Magnitudes and Colors, Sorted by Infrared Spectral Type

\begin{tabular}{|c|c|c|c|c|c|c|c|c|c|c|}
\hline Name & Type $^{\text {a }}$ & $M_{J}$ & $i-z$ & $z$ & $z-J$ & $Z-J$ & $J$ & $J-K$ & $J-H$ & $H-K$ \\
\hline 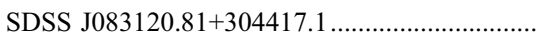 & {$[\mathrm{M}-\mathrm{L} 2]$} & $\ldots$ & 1.77 & 19.68 & 2.23 & $\ldots$ & 17.45 & 1.10 & 0.56 & 0.54 \\
\hline 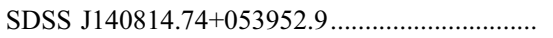 & {$[\mathrm{M}-\mathrm{L} 2]$} & $\ldots$ & 1.77 & 18.71 & 2.22 & $\ldots$ & 16.49 & 1.00 & 0.54 & 0.46 \\
\hline SDSS J162441.00+444145.8 & {$[\mathrm{M}-\mathrm{L} 3]$} & $\ldots$ & $\ldots$ & 20.07 & 2.51 & $\ldots$ & 17.56 & 1.10 & 0.68 & 0.42 \\
\hline 2 MASS J03454316+2540233 ${ }^{\mathrm{b}} \ldots$ & $\mathrm{L} 1 \pm 1$ & 11.69 & $\ldots$ & $\ldots$ & $\ldots$ & $\ldots$ & 13.84 & 1.18 & 0.63 & 0.54 \\
\hline 2MASS J07464256+2000321AB ... & L1 & 11.21 & 1.80 & 14.30 & 2.66 & 1.47 & 11.64 & 1.21 & 0.63 & 0.58 \\
\hline 2MASS J14392836+1929149_.......................... & $\mathrm{L} 1^{\mathrm{c}}$ & 11.87 & $\ldots$ & $\ldots$ & $\ldots$ & $\ldots$ & 12.66 & 1.19 & 0.61 & 0.58 \\
\hline 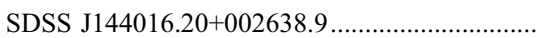 & $\mathrm{L} 1^{\mathrm{d}}$ & $\ldots$ & 1.90 & 18.75 & 2.82 & $\ldots$ & 15.93 & 1.29 & 0.70 & 0.59 \\
\hline 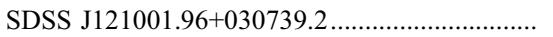 & $\mathrm{L} 1.5 \pm 1$ & $\ldots$ & $\ldots$ & 19.79 & 2.52 & $\ldots$ & 17.27 & 1.24 & 0.69 & 0.55 \\
\hline 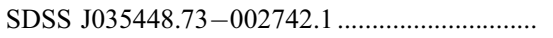 & $\mathrm{L} 2 \pm 1$ & $\ldots$ & 2.02 & 19.61 & 2.47 & $\ldots$ & 17.14 & 1.19 & 0.68 & 0.51 \\
\hline 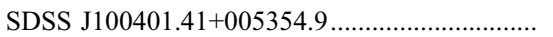 & $\mathrm{L} 2 \pm 1$ & $\ldots$ & 2.05 & 19.76 & 2.36 & $\ldots$ & 17.40 & 1.16 & 0.58 & 0.58 \\
\hline 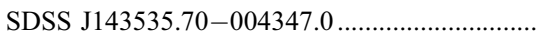 & $\mathrm{L} 2.5$ & 12.44 & 1.84 & 19.02 & 2.61 & $\ldots$ & 16.41 & 1.29 & 0.73 & 0.56 \\
\hline 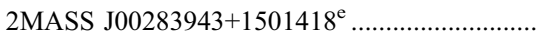 & $\mathrm{L}^{\mathrm{d}}$ & $\ldots$ & 2.19 & 19.52 & 3.03 & 2.01 & 16.49 & 1.95 & 1.01 & 0.94 \\
\hline DENIS-P J1058.7-1548 ${ }^{\mathrm{b}, \mathrm{f}} \ldots \ldots \ldots$ & L3 & 12.93 & $\ldots$ & $\ldots$ & $\ldots$ & 1.64 & 14.12 & 1.57 & 0.84 & 0.74 \\
\hline 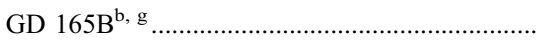 & $\mathrm{L} 3 \pm 2$ & 13.14 & $\ldots$ & $\ldots$ & $\ldots$ & $\ldots$ & 15.64 & 1.55 & 0.89 & 0.66 \\
\hline Kelu- $1^{\mathrm{b}, \mathrm{h}} \ldots \ldots$ & $\mathrm{L} 3 \pm 1$ & 11.88 & $\ldots$ & $\ldots$ & $\ldots$ & 1.77 & 13.23 & 1.45 & 0.78 & 0.67 \\
\hline SDSS J020735.60+135556.3 & $\mathrm{L} 3 \pm 1.5$ & $\ldots$ & 1.78 & 18.06 & 2.79 & $\ldots$ & 15.27 & 1.46 & 0.82 & 0.64 \\
\hline 2MASS J22244381-0158521 ........................... & $\mathrm{L} 3.5$ & 13.59 & $\ldots$ & $\ldots$ & $\ldots$ & $\ldots$ & 13.89 & 1.91 & 1.05 & 0.86 \\
\hline 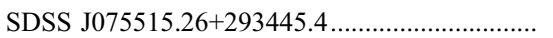 & $\mathrm{L} 3.5 \pm 1$ & $\ldots$ & 2.33 & 19.40 & 2.69 & $\ldots$ & 16.71 & 1.39 & 0.77 & 0.62 \\
\hline 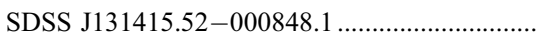 & $\mathrm{L} 3.5 \pm 1.5$ & $\ldots$ & 2.00 & 19.63 & 3.11 & $\ldots$ & 16.52 & 1.22 & 0.68 & 0.55 \\
\hline 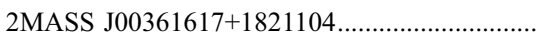 & $\mathrm{L} 4 \pm 1$ & 12.61 & $\ldots$ & $\ldots$ & $\ldots$ & 1.81 & 12.30 & 1.26 & 0.66 & 0.60 \\
\hline 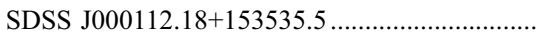 & $\mathrm{L} 4 \pm 1$ & $\ldots$ & 1.74 & 18.55 & 3.26 & $\ldots$ & 15.29 & 1.77 & 0.89 & 0.88 \\
\hline 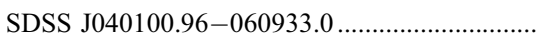 & $\mathrm{L} 4 \pm 1.5$ & $\ldots$ & $\ldots$ & 20.19 & 2.81 & $\ldots$ & 17.38 & 1.67 & 0.99 & 0.68 \\
\hline 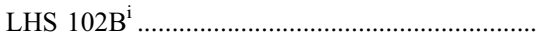 & $\mathrm{L} 4.5$ & 13.16 & $\ldots$ & $\ldots$ & $\ldots$ & 1.61 & 13.06 & 1.70 & 0.92 & 0.78 \\
\hline 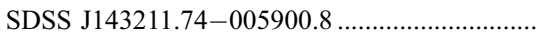 & L4.5 & $\ldots$ & $\ldots$ & 19.62 & 2.63 & $\ldots$ & 16.99 & 1.54 & 0.85 & 0.69 \\
\hline 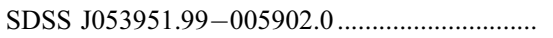 & L5 & 13.26 & 2.24 & 16.78 & 2.93 & 1.75 & 13.85 & 1.45 & 0.81 & 0.64 \\
\hline 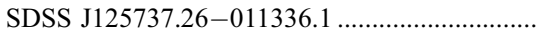 & L5 & 13.86 & 2.14 & 18.56 & 2.92 & $\ldots$ & 15.64 & 1.58 & 0.96 & 0.62 \\
\hline 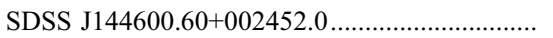 & L5 & 13.85 & 2.30 & 18.48 & 2.92 & $\ldots$ & 15.56 & 1.76 & 0.97 & 0.79 \\
\hline 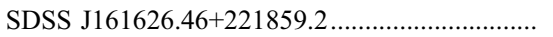 & $\mathrm{L} 5 \pm 2$ & $\ldots$ & $\ldots$ & 20.33 & 2.80 & $\ldots$ & 17.53 & 1.88 & 1.03 & 0.85 \\
\hline 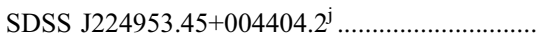 & $\mathrm{L} 5 \pm 1.5^{\mathrm{k}}$ & $\ldots$ & 2.16 & 19.42 & 2.95 & 1.77 & 16.47 & 2.05 & 1.11 & 0.94 \\
\hline 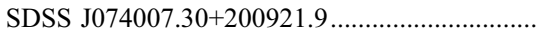 & [L3-8] & $\ldots$ & $\ldots$ & 19.78 & 3.11 & $\ldots$ & 16.67 & 1.56 & 0.85 & 0.71 \\
\hline 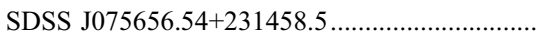 & [L3-8] & $\ldots$ & $\ldots$ & 19.82 & 3.02 & $\ldots$ & 16.80 & 1.80 & 0.98 & 0.82 \\
\hline 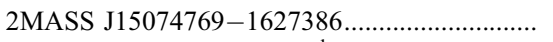 & L5.5 & 13.37 & $\ldots$ & $\ldots$ & $\ldots$ & $\ldots$ & 12.70 & 1.41 & 0.80 & 0.61 \\
\hline 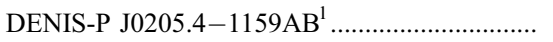 & $\mathrm{L} 5.5 \pm 2^{\mathrm{d}}$ & 12.95 & $\ldots$ & $\ldots$ & $\ldots$ & 1.58 & 14.43 & 1.44 & 0.82 & 0.62 \\
\hline 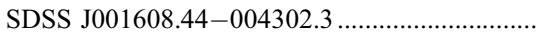 & L5.5 & $\ldots$ & 1.77 & 19.34 & 3.00 & $\ldots$ & 16.34 & 1.82 & 1.00 & 0.82 \\
\hline 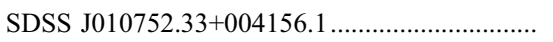 & $\mathrm{L} 5.5^{\mathrm{d}}$ & 14.78 & 2.83 & 18.70 & 2.95 & 1.58 & 15.75 & 2.17 & 1.19 & 0.98 \\
\hline 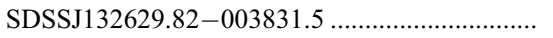 & L5.5 & 14.70 & 2.63 & 19.05 & 2.84 & $\ldots$ & 16.21 & 2.04 & 1.11 & 0.93 \\
\hline 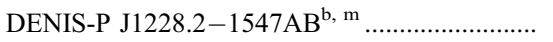 & $\mathrm{L} 6 \pm 2$ & 12.75 & $\ldots$ & $\ldots$ & $\ldots$ & 1.73 & 14.28 & 1.57 & 0.88 & 0.69 \\
\hline 2MASS J08251968+2115521 ............................. & $\mathrm{L}^{\mathrm{d}}$ & 14.76 & $\ldots$ & $\ldots$ & $\ldots$ & 1.73 & 14.89 & 1.96 & 1.08 & 0.88 \\
\hline 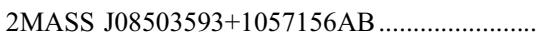 & $\mathrm{L} 6^{\mathrm{c}}$ & 13.85 & $\ldots$ & $\ldots$ & $\ldots$ & 1.95 & 16.20 & 1.85 & 0.99 & 0.86 \\
\hline 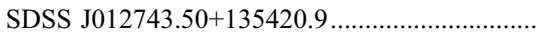 & $\mathrm{L} 6 \pm 1$ & $\ldots$ & 2.65 & 19.62 & 2.91 & $\ldots$ & 16.71 & 1.62 & 0.87 & 0.75 \\
\hline SDSS J104625.76+042441.0 & L6 & $\ldots$ & 2.64 & 19.74 & 2.77 & $\ldots$ & 16.97 & 1.62 & 0.94 & 0.68 \\
\hline 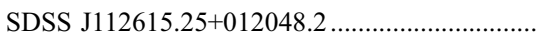 & L6 & $\ldots$ & $\ldots$ & 19.79 & 3.11 & $\ldots$ & 16.68 & 1.64 & 0.87 & 0.77 \\
\hline 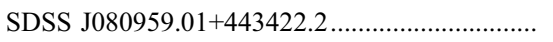 & {$[\mathrm{L} 5-8]$} & $\ldots$ & 2.54 & 19.28 & 2.91 & $\ldots$ & 16.37 & 2.06 & 1.12 & 0.94 \\
\hline 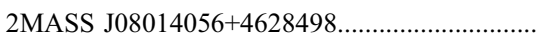 & $\mathrm{L} 6.5^{\mathrm{c}}$ & $\ldots$ & 2.50 & 18.78 & 2.57 & $\ldots$ & 16.21 & 1.63 & 0.90 & 0.73 \\
\hline 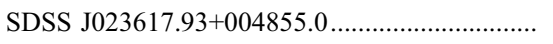 & L6.5 & $\ldots$ & 2.87 & 18.80 & 2.79 & 1.53 & 16.01 & 1.47 & 0.85 & 0.62 \\
\hline 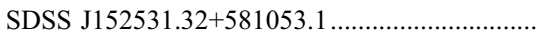 & $\mathrm{L} 6.5 \pm 1$ & $\ldots$ & $\ldots$ & 19.75 & 2.85 & $\ldots$ & 16.90 & 1.47 & 0.77 & 0.70 \\
\hline 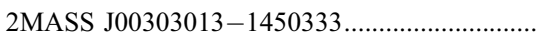 & $\mathrm{L} 7^{\mathrm{c}}$ & 14.26 & $\ldots$ & $\ldots$ & $\ldots$ & 1.74 & 16.39 & 1.90 & 1.02 & 0.88 \\
\hline 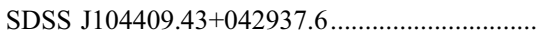 & L7 & $\ldots$ & 2.94 & 18.73 & 2.89 & $\ldots$ & 15.84 & 1.52 & 0.87 & 0.65 \\
\hline 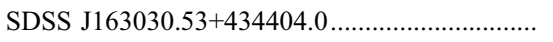 & $\mathrm{L} 7 \pm 1.5$ & $\ldots$ & 2.65 & 19.45 & 2.97 & $\ldots$ & 16.48 & 1.78 & 0.97 & 0.81 \\
\hline 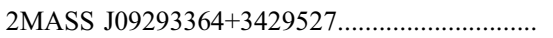 & L7.5 & $\ldots$ & $\ldots$ & 19.52 & 2.83 & 1.69 & 16.69 & 1.96 & 1.07 & 0.89 \\
\hline 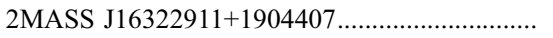 & L7.5 & 14.83 & 3.20 & 18.58 & 2.81 & 1.75 & 15.77 & 1.80 & 1.09 & 0.71 \\
\hline 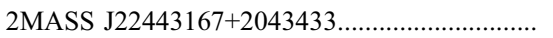 & $\mathrm{L} 7.5 \pm 2$ & $\ldots$ & $\ldots$ & $\ldots$ & $\ldots$ & $\ldots$ & 16.33 & 2.43 & 1.27 & 1.16 \\
\hline 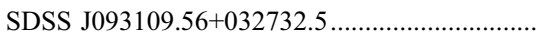 & $\mathrm{L} 7.5 \pm 1.5$ & $\ldots$ & 2.72 & 19.28 & 2.68 & $\ldots$ & 16.60 & 1.07 & 0.51 & 0.56 \\
\hline 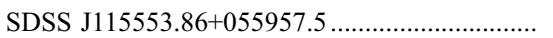 & L7.5 & $\ldots$ & 2.81 & 18.45 & 2.82 & $\ldots$ & 15.63 & 1.54 & 0.89 & 0.65 \\
\hline 2 MASS J15232263+3014562 ${ }^{\mathrm{n}} \ldots$ & L8 & 14.63 & $\ldots$ & $\ldots$ & $\ldots$ & 1.65 & 15.95 & 1.60 & 0.90 & 0.70 \\
\hline SDSS J003259.36+141036.6 & L8 & 13.98 & $\ldots$ & 19.40 & 2.82 & 1.67 & 16.58 & 1.59 & 0.92 & 0.67 \\
\hline 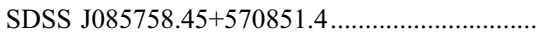 & $\mathrm{L} 8 \pm 1$ & $\ldots$ & 2.98 & 17.77 & 2.97 & 1.72 & 14.80 & 1.86 & 1.00 & 0.86 \\
\hline 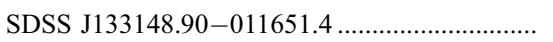 & $\mathrm{L} 8 \pm 2.5^{\mathrm{k}}$ & $\ldots$ & 2.42 & 18.14 & 2.82 & $\ldots$ & 15.32 & 1.25 & 0.67 & 0.58 \\
\hline 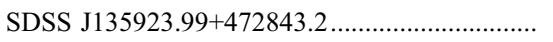 & L8.5 & $\ldots$ & $\ldots$ & 19.76 & 2.81 & $\ldots$ & 16.95 & 1.61 & 0.95 & 0.66 \\
\hline 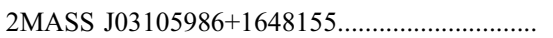 & L9 & $\ldots$ & $\ldots$ & $\ldots$ & $\ldots$ & $\ldots$ & 15.88 & 1.70 & 0.97 & 0.73 \\
\hline 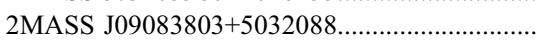 & $\mathrm{L} 9 \pm 1^{\mathrm{k}}$ & $\ldots$ & 2.84 & 17.22 & 2.82 & $\ldots$ & 14.40 & 1.51 & 0.86 & 0.65 \\
\hline 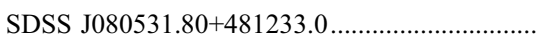 & $\mathrm{L} 9 \pm 1.5^{\mathrm{k}}$ & $\ldots$ & 2.20 & 17.62 & 3.01 & $\ldots$ & 14.61 & 1.10 & 0.60 & 0.50 \\
\hline 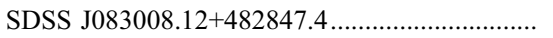 & $\mathrm{L} 9 \pm 1$ & 14.64 & 3.14 & 18.08 & 2.86 & 1.64 & 15.22 & 1.54 & 0.82 & 0.72 \\
\hline 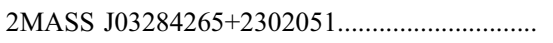 & $\mathrm{L} 9.5^{\mathrm{k}}$ & 13.95 & $\ldots$ & $\ldots$ & $\ldots$ & 1.71 & 16.35 & 1.48 & 0.88 & 0.60 \\
\hline 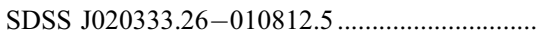 & L9.5 & $\ldots$ & $\ldots$ & 20.36 & 2.53 & $\ldots$ & 17.83 & 1.65 & 0.96 & 0.69 \\
\hline
\end{tabular}


TABLE 9-Continued

\begin{tabular}{|c|c|c|c|c|c|c|c|c|c|c|}
\hline Name & Type $^{\mathrm{a}}$ & $M_{J}$ & $i-z$ & $z$ & $z-J$ & $Z-J$ & $J$ & $J-K$ & $J-H$ & $H-K$ \\
\hline SDSS J085234.90+472035.0 & $\mathrm{L} 9.5 \pm 1$ & $\ldots$ & 3.09 & 18.90 & 2.77 & $\ldots$ & 16.13 & 1.51 & 0.92 & 0.59 \\
\hline SDSS J103026.78+021306.4 …....................... & $\mathrm{L} 9.5 \pm 1$ & $\ldots$ & $\ldots$ & 19.94 & 2.84 & $\ldots$ & 17.10 & 1.43 & 0.83 & 0.60 \\
\hline 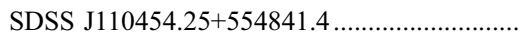 & $\mathrm{L} 9.5 \pm 1.5$ & $\ldots$ & $\ldots$ & 19.94 & 2.66 & $\ldots$ & 17.28 & 0.94 & 0.55 & 0.39 \\
\hline SDSS J204749.61-071818.3 …….................... & $\mathrm{L} 9.5 \pm 1$ & & $\ldots$ & 19.74 & 3.04 & $\ldots$ & 16.70 & 1.36 & 0.82 & 0.54 \\
\hline SDSS J042348.57-041403.5 ............................ & $\mathrm{T} 0^{\mathrm{k}}$ & 13.39 & 2.89 & 17.29 & 2.99 & 1.68 & 14.30 & 1.34 & 0.79 & 0.55 \\
\hline 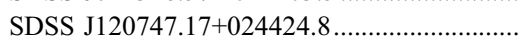 & $\mathrm{T} 0^{\mathrm{k}}$ & $\ldots$ & 3.06 & 18.41 & 3.03 & $\ldots$ & 15.38 & 1.22 & 0.75 & 0.47 \\
\hline 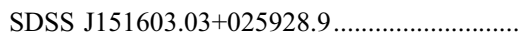 & $\mathrm{T} 0 \pm 1.5$ & & $\ldots$ & 19.89 & 3.01 & . & 16.88 & 1.53 & 0.81 & 0.72 \\
\hline 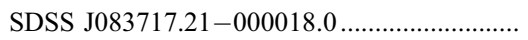 & $\mathrm{T} 0.5$ & 14.54 & $\ldots$ & 20.06 & 3.16 & 1.69 & 16.90 & 0.92 & 0.69 & 0.23 \\
\hline 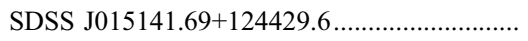 & $\mathrm{T} 1 \pm 1$ & 14.60 & $\ldots$ & 19.45 & 3.20 & 1.84 & 16.25 & 1.07 & 0.71 & 0.36 \\
\hline 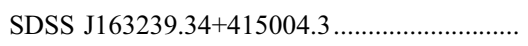 & $\mathrm{T} 1 \pm 1$ & $\ldots$ & $\ldots$ & 20.35 & 3.48 & $\ldots$ & 16.87 & 0.68 & 0.45 & 0.23 \\
\hline 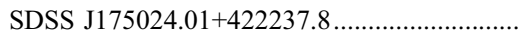 & $\mathrm{T} 1$ & $\ldots$ & $\ldots$ & 19.38 & 3.26 & $\ldots$ & 16.12 & 0.81 & 0.55 & 0.26 \\
\hline 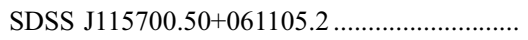 & $\mathrm{T} 1.5$ & $\ldots$ & $\ldots$ & 20.20 & 3.11 & $\ldots$ & 17.09 & 1.09 & 0.64 & 0.45 \\
\hline SDSS J075840.33+324723.4 & $\mathrm{T} 2 \pm 1$ & $\ldots$ & 3.96 & 17.96 & 3.18 & $\ldots$ & 14.78 & 0.91 & 0.57 & 0.34 \\
\hline 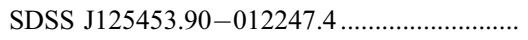 & $\mathrm{T} 2$ & 14.00 & 4.22 & 18.03 & 3.37 & 1.74 & 14.66 & 0.82 & 0.53 & 0.29 \\
\hline 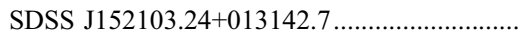 & $\mathrm{T} 2$ & $\ldots$ & $\ldots$ & 19.57 & 3.51 & $\ldots$ & 16.06 & 0.58 & 0.43 & 0.15 \\
\hline 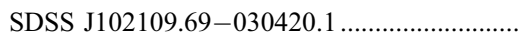 & $\mathrm{T} 3$ & 13.62 & $\ldots$ & 19.33 & 3.45 & 1.78 & 15.88 & 0.62 & 0.47 & 0.15 \\
\hline 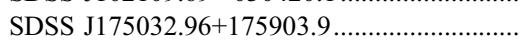 & $\mathrm{T} 3.5$ & 13.94 & $\ldots$ & 19.63 & 3.49 & $\ldots$ & 16.14 & 0.12 & 0.20 & -0.08 \\
\hline 2MASS J22541892+3123498_........................... & $\mathrm{T} 4$ & $\ldots$ & $\ldots$ & $\ldots$ & $\ldots$ & $\ldots$ & 15.01 & -0.02 & 0.06 & -0.08 \\
\hline 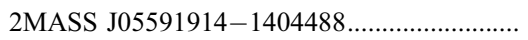 & $\mathrm{T} 4.5$ & 13.50 & $\ldots$ & $\ldots$ & $\ldots$ & 1.98 & 13.57 & -0.16 & -0.07 & -0.09 \\
\hline 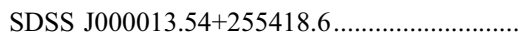 & $\mathrm{T} 4.5$ & $\ldots$ & $\ldots$ & 18.48 & 3.75 & $\ldots$ & 14.73 & -0.09 & -0.01 & -0.08 \\
\hline 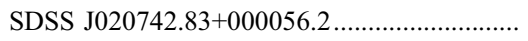 & $\mathrm{T} 4.5$ & 14.34 & $\ldots$ & 20.11 & 3.48 & 2.08 & 16.63 & 0.01 & -0.03 & 0.04 \\
\hline 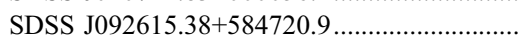 & $\mathrm{T} 4.5$ & $\ldots$ & $\ldots$ & 19.01 & 3.54 & $\ldots$ & 15.47 & -0.03 & 0.05 & -0.08 \\
\hline 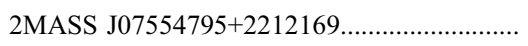 & $\mathrm{T} 5^{\circ}$ & $\ldots$ & $\ldots$ & 19.12 & 3.66 & $\ldots$ & 15.46 & -0.40 & -0.24 & -0.16 \\
\hline 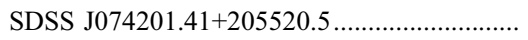 & $\mathrm{T} 5$ & $\ldots$ & $\ldots$ & 19.28 & 3.68 & $\ldots$ & 15.60 & -0.46 & -0.35 & -0.11 \\
\hline 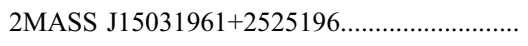 & $\mathrm{T} 5.5^{\mathrm{o}}$ & $\ldots$ & $\ldots$ & $\ldots$ & $\ldots$ & $\ldots$ & 13.55 & -0.44 & -0.35 & -0.09 \\
\hline 2MASS J15344984-2952274AB ..................... & $\mathrm{T} 5.5^{\mathrm{o}}$ & 13.93 & $\ldots$ & $\ldots$ & $\ldots$ & $\ldots$ & 14.60 & -0.31 & -0.14 & -0.17 \\
\hline 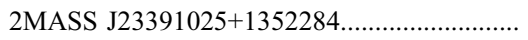 & $\mathrm{T} 5.5$ & $\ldots$ & $\ldots$ & $\ldots$ & $\ldots$ & $\ldots$ & 15.81 & -0.36 & -0.19 & -0.17 \\
\hline SDSS J074149.15+235127.5 & $\mathrm{T} 5.5$ & $\ldots$ & $\ldots$ & 19.65 & 3.78 & $\ldots$ & 15.87 & -0.35 & -0.25 & -0.10 \\
\hline 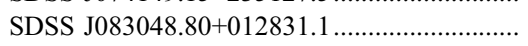 & $\mathrm{T} 5.5$ & $\ldots$ & $\ldots$ & 19.59 & 3.60 & $\ldots$ & 15.99 & -0.39 & -0.18 & -0.21 \\
\hline 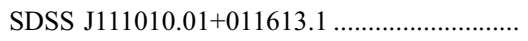 & T5.5 & $\ldots$ & $\ldots$ & 19.64 & 3.52 & $\ldots$ & 16.12 & 0.07 & -0.10 & 0.17 \\
\hline 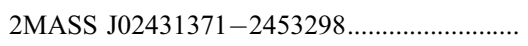 & $\mathrm{T} 6^{\mathrm{o}}$ & 14.99 & $\ldots$ & $\ldots$ & $\ldots$ & 1.85 & 15.13 & -0.21 & -0.26 & 0.05 \\
\hline 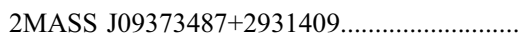 & $\mathrm{T} 6$ & 15.35 & $\ldots$ & $\ldots$ & $\ldots$ & 1.72 & 14.29 & -1.10 & -0.38 & -0.72 \\
\hline 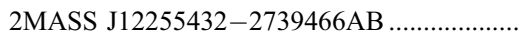 & T6 & 14.25 & $\ldots$ & $\ldots$ & $\ldots$ & 1.89 & 14.88 & -0.40 & -0.29 & -0.11 \\
\hline 2MASS J23565477-1553111 .............................. & $\mathrm{T} 6^{\circ}$ & 14.67 & $\ldots$ & $\ldots$ & $\ldots$ & 2.12 & 15.48 & -0.25 & -0.22 & -0.03 \\
\hline Gl 229B $\mathrm{B}^{\mathrm{p}}$ & $\mathrm{T} 6 \pm 1$ & 15.20 & $\ldots$ & $\ldots$ & $\ldots$ & 2.17 & 14.01 & -0.35 & -0.35 & 0.00 \\
\hline 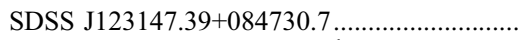 & T6 & & $\ldots$ & 18.94 & 3.80 & . & 15.14 & -0.32 & -0.26 & -0.06 \\
\hline SDSS J134646.45-003150.4 $4^{\mathrm{b}} \ldots \ldots \ldots \ldots \ldots \ldots \ldots \ldots$ & T6 & 14.69 & $\ldots$ & 19.29 & 3.80 & 2.24 & 15.49 & -0.24 & -0.35 & 0.11 \\
\hline SDSS J162414.37+002915.6 & $\mathrm{T} 6$ & 14.99 & $\ldots$ & 19.02 & 3.82 & 2.12 & 15.20 & -0.41 & -0.28 & -0.13 \\
\hline 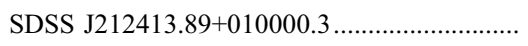 & T6 & $\ldots$ & $\ldots$ & 19.71 & 3.83 & $\ldots$ & 15.88 & -0.19 & -0.24 & 0.05 \\
\hline 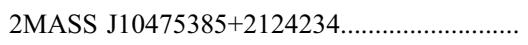 & $\mathrm{T} 6.5$ & 15.43 & $\ldots$ & $\ldots$ & $\ldots$ & 1.93 & 15.46 & -0.74 & -0.37 & -0.37 \\
\hline 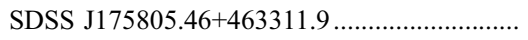 & $\mathrm{T} 7$ & $\ldots$ & $\ldots$ & 19.67 & 3.81 & $\ldots$ & 15.86 & -0.26 & -0.34 & 0.08 \\
\hline 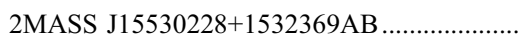 & $\mathrm{T} 7.5^{\mathrm{o}}$ & $\ldots$ & $\ldots$ & $\ldots$ & $\ldots$ & $\ldots$ & 15.34 & -0.60 & -0.42 & -0.18 \\
\hline 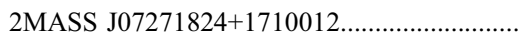 & $\mathrm{T} 8$ & 15.40 & $\ldots$ & $\ldots$ & $\ldots$ & 1.98 & 15.19 & -0.50 & -0.48 & -0.02 \\
\hline 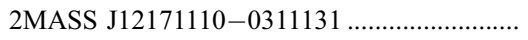 & $\mathrm{T} 8$ & 15.41 & $\ldots$ & 19.18 & 3.62 & 2.00 & 15.56 & -0.36 & -0.42 & 0.06 \\
\hline G1 570D & $\mathrm{T} 8$ & 15.97 & $\ldots$ & $\ldots$ & $\ldots$ & 1.92 & 14.82 & -0.70 & -0.46 & -0.24 \\
\hline 2MASS J04151954-0935066........................... & T9 & 16.53 & $\ldots$ & $\ldots$ & $\ldots$ & 1.98 & 15.32 & -0.51 & -0.38 & -0.13 \\
\hline
\end{tabular}

a Spectral types in square brackets estimated from colors.

b $J H K$ magnitudes synthesized from flux calibrated spectrum.

c Spectral type from Kirkpatrick et al. 2000.

d Optical spectral type later than infrared type by greater than one subclass.

e 2MASS J0028+1501 may be variable at the $5 \%$ level; measured $J / H / K$ of $16.52(0.03) / 15.42(0.03) / 14.63(0.05)$ on $20001119,16.65(0.05) / 15.56(0.05) /$ 14.57(0.05) on 20010114, 16.45(0.03)/15.54(0.03)/14.51(0.03) on 20011124 and 16.44(0.03)/15.44(0.03)/14.53(0.03) on 20020108.

${ }^{f}$ DENIS-P J1058.7-1548=2MASS J10584787-1548172.

g GD 165B=2MASS J14243909+0917104.

${ }^{\mathrm{h}}$ Kelu-1=2MASS J13054019-2541059.

${ }^{\mathrm{i}}$ LHS 102B=2MASS J00043484-4044058.

j SDSS J2249+0044 may be variable at the $10 \%$ level; measured $J / H / K$ of $16.58(0.03) / 15.39(0.03) / 14.46(0.03)$ on $20001119,16.46(0.05) / 15.42(0.05) /$ $14.43(0.05)$ on $20010116,16.36(0.03) / 15.30(0.03) / 14.38(0.03)$ on 20020108.

${ }^{\mathrm{k}}$ Optical spectral type earlier than infrared type by greater than one subclass.

${ }^{1}$ DENIS-P J0205.4-1159AB=2MASS J02052940-1159296AB.

${ }^{m}$ DENIS-P J1228.2-1547AB=2MASS J12281523-1547342AB.

n 2MASS J15232263+3014562=Gl 584C.

${ }^{\circ}$ Spectral type from Burgasser et al. 2002 or typed by us from Burgasser et al. data.

p $Z J H K$ magnitudes synthesized from flux calibrated spectrum.

${ }^{\mathrm{q}}$ Gl 570D=2MASS J14571496-2121477. 
TABLE 10

SDSS $r$ Magnitudes for Detected Objects in Table 9

\begin{tabular}{|c|c|c|}
\hline Name & $r$ & $\sigma_{r}$ \\
\hline 2MASS J0746+2000AB.. & 18.65 & 0.01 \\
\hline 2MASS J0755+2212.. & 22.27 & 0.23 \\
\hline 2MASS J0908+5032.. & 22.43 & 0.17 \\
\hline SDSS J0001+1535 .... & 23.02 & 0.22 \\
\hline SDSS J0207+1355 .... & 22.37 & 0.14 \\
\hline 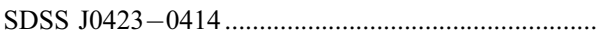 & 22.82 & 0.22 \\
\hline SDSS J0539-0059 ... & 21.42 & 0.08 \\
\hline SDSS J0755+2934 ..... & 22.96 & 0.29 \\
\hline 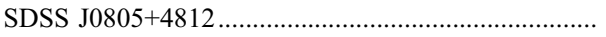 & 22.79 & 0.27 \\
\hline SDSS J1257-0113. & 22.68 & 0.27 \\
\hline SDSS J1314-0008 .... & 23.32 & 0.27 \\
\hline 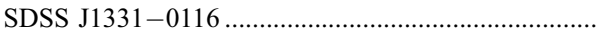 & 22.91 & 0.26 \\
\hline SDSS J1440+0026...... & 22.92 & 0.19 \\
\hline SDSS J1446+0024 & 23.20 & 0.23 \\
\hline
\end{tabular}

Note. - The $r$ magnitude for zero flux $=25.1$.

within which the condensates are found. They describe their limits to be the points at which the atmosphere is cool enough for condensation but hot enough that the condensates are small enough to remain suspended in the atmosphere and are less prone to sedimentation. The temperature domain in which the cloud is formed depends on the details of the model used, but is around $\sim 1500-1700 \mathrm{~K}$ (Ackerman \& Marley 2001) or 1800-2000 K (Tsuji \& Nakajima 2003). In the T dwarfs the cloud layer lies near the base or below the wavelengthdependent photosphere and plays a smaller role in determining the observed flux distribution.

The second and later chemical change that occurs in these high-pressure, low-temperature atmospheres is the formation of additional molecular species in the photosphere, most importantly $\mathrm{CH}_{4}$. $\mathrm{CO}$ and $\mathrm{H}_{2} \mathrm{O}$ are abundant in $\mathrm{M}$ dwarf atmospheres, but by mid $\mathrm{L}$ the abundance of $\mathrm{CH}_{4}$ becomes significant at the expense of CO (Noll et al. 2000). At moderate spectral resolution $\mathrm{CH}_{4}$ absorption is not seen in the near-infrared until temperatures drop to those of the late $\mathrm{L}$ dwarfs, at which point $K$-band $\mathrm{CH}_{4}$ features are detectable, and at $\mathrm{T} 0$ (by definition, G02) $\mathrm{CH}_{4}$ absorptions are seen at both $H$ and $K$. The increasing $\mathrm{CH}_{4}$ absorption largely accounts for the increasingly blue $J H K$ colors of the T dwarfs with later spectral type, more than compensating for the reddening due to the decreasing effective temperature. For dwarfs of type T5 and later, pressure-induced $\mathrm{H}_{2}$ becomes a significant opacity source. This opacity depresses the flux in the $K$ band, and to a lesser extent the $H$ band, and also contributes to the blue nearinfrared colors. For a useful summary of the important molecular species and the wavelength ranges in which they are observed, see Figure 15 of Burrows et al. (2001).

\subsection{Clouds, Molecules, and Classification Schemes}

Spectral classification schemes for $\mathrm{L}$ and $\mathrm{T}$ dwarfs have been developed using both the red and the near-infrared spectral regions. In the late 1990s Kirkpatrick et al. (1999) and Martín et al. (1999b) developed schemes using the strengths of various absorption features and pseudocontinuum slopes seen in optical spectra to classify the L dwarfs. A few years later Burgasser et al. (2002a) and G02 presented schemes using the strengths of the near-infrared molecular absorption bands to classify the $\mathrm{T}$ dwarfs, and in the case of $\mathrm{G} 02$, L dwarfs also. While the Burgasser et al. (2002a) and G02 schemes for $\mathrm{T}$ dwarfs give results in very close agreement, the G02 scheme for L dwarfs can give results that differ by as much as 2.5 subclasses from those given by the Kirkpatrick et al. (1999) scheme, suggesting that there are significant differences in the optical and infrared classification of L dwarfs. Dwarfs in our sample whose optical and infrared spectral classes differ by more than one subclass are identified in Table 9. Some of the scatter is due to the small differences in the infrared indices from one subtype to the next, combined with measurement errors. However, the differences in optical and infrared spectral types are not entirely random. Where there are differences, G02 generally assign earlier spectral types to those objects that are redder than average in $J-K$ and later types to those that are bluer (Stephens 2001, Fig. 7.5).

Models of $\mathrm{L}$ and $\mathrm{T}$ dwarf atmospheres give some insight into the variations seen among the spectral indices. Generally speaking, the spectra of $\mathrm{L}$ and $\mathrm{T}$ dwarfs are less sensitive to the effects of cloud decks in the $0.7-1.0 \mu \mathrm{m}$ spectral region than at wavelengths longer than $1 \mu \mathrm{m}$. This is because, for effective temperatures corresponding to the earliest $\mathrm{L}$ types, optical depth unity in the far-red is reached below the cloud deck, but as the cloud is still fairly optically thin it does not substantially influence the red spectrum. At lower effective temperatures the clouds place a "floor" on the region from which the emergent flux arises, but because of the large opacity in the far-red due to initially refractory diatomics and water and later to $\mathrm{K}$ I and $\mathrm{Na}$ I resonance line absorption, most of the outgoing red flux arises from above the cloud decks. Thus, for dwarfs with effective temperature cooler than about $1800 \mathrm{~K}$ (types $\sim$ L3 and later, Leggett et al. 2002a, 2002b; Golimowski et al. 2004) slight changes in the cloud deck optical depth have little effect on the emergent red spectrum.

In the near-infrared (particularly the $Z$ and the $J$ bands) the windows between the molecular bands of water and other opacity sources allow flux to emerge from very deep in the atmosphere. In these regions the opacity floor imposed by the clouds substantially alters the depth to which one can see into the atmosphere (see Fig. 7 of Ackerman \& Marley 2001 and Fig. 4 of Marley et al. 2002). Thus, for dwarfs with $T_{\text {eff }}$ in the range from about 1800 to $1500 \mathrm{~K}$ (roughly L3 to L7), slight changes in the cloud profile substantially alter the nearinfrared spectrum.

This proposed atmospheric structure implies that spectral typing schemes for mid to late $\mathrm{L}$ dwarfs that rely on far-red spectra (e.g., Kirkpatrick et al. 1999) tend to be less sensitive to the vertical distribution of condensates in the atmosphere than schemes that rely on near-infrared spectra or spectral indices (G02). Stephens $(2001,2003)$ considered the boundaries of the regions employed in the G02 spectral typing system and found that the flux in the G02 bandpasses usually originates from within the cloud decks. For the earliest L dwarfs, cloud opacity is not significant, but for the mid L dwarfs, the G02 $1.5 \mu \mathrm{m}$ water index can be a more sensitive indicator of cloud optical depth than of effective temperature. At the same time, the $2.2 \mu \mathrm{m}$ methane index is more sensitive to $T_{\text {eff }}$ than to cloud properties, since this spectral region is more opaque and optical depth unity is reached higher in the atmosphere. However, the classification system of G02 relies heavily on the $1.5 \mu \mathrm{m}$ index, as it is the only infrared index that covers the entire L spectral type range. This index undergoes a much larger change through the $\mathrm{L}$ sequence than does the other useful infrared index, $\mathrm{CH}_{4}-\mathrm{K}$. Note that $\mathrm{G} 02$ do not claim that defining the spectral type is equivalent to measuring the effective temperature; they use a simple classification scheme based on spectral 
appearance in which the effects of gravity and clouds are not separated from those of temperature.

This larger sample of dwarfs also suggests some inconsistency in the infrared classification of late L dwarfs. While the G02 scheme provides excellent internal consistency for $\mathrm{T}$ dwarfs, the $\mathrm{H}_{2} \mathrm{O} 1.5 \mu \mathrm{m}$ index tends to give a later spectral type than does the $\mathrm{CH}_{4} 2.2 \mu \mathrm{m}$ index for dwarfs in the range L5-L9.5. This tendency was not apparent in the smaller G02 sample; the results for the present larger sample suggest that an adjustment of the flux ratio definitions as a function of spectral type for $\mathrm{H}_{2} \mathrm{O} 1.5 \mu \mathrm{m}$ and $\mathrm{CH}_{4} 2.2 \mu \mathrm{m}$ in the L5-L9.5 range could give better internal consistency.

\subsection{Observed Color as a Function of Spectral Type}

Figure 3 plots, for the final sample presented in Table 9, various colors against spectral type (determined by the G02 scheme apart from four L dwarfs classified optically; see Table 9); typical error bars are shown. Only those dwarfs with types determined from their spectra are shown. Clouds strongly affect both colors and spectral types of mid L dwarfs classified from the near-infrared indices, as discussed in $\S \S 5.1$ and 5.2. This effect can be seen in Figure 3, where the spread in the $Z$ through $K$ colors is greatest from L3 to L7.5, just when the clouds in the detectable atmosphere are expected to be most optically thick. The overall conclusion is that color cannot be used as an (infrared) spectral type indicator for $\mathrm{L}$ dwarfs. For $\mathrm{T}$ dwarfs, $z-J$ and $J-H$ appear to be reasonable indicators of type. Given improved sensitivity, $i-z$ may also be a useful T-type indicator. Note, however, that $i-z$ is expected to turn blueward at $T_{\text {eff }} \lesssim 600 \mathrm{~K}$ as the $\mathrm{Na}, \mathrm{K}$, and other alkalis condense into solids, and the opacity of the $\mathrm{Na}$ and $\mathrm{K}$ lines falls (Burrows et al. 2002; Marley et al. 2002). The late $\mathrm{T}$ dwarfs show significant scatter in their $H-K$ and $J-K$ colors. This can be understood in terms of the onset of pressure-induced $\mathrm{H}_{2}$ absorption, which is very gravity sensitive (Borysow, Jorgensen, \& Zheng 1997). As described in $\S 5.6$, we can interpret the observed spread in $H-K$ as a range in surface gravity for the field $\mathrm{T}$ dwarf population.

The $Z-J$ colors of the mid $\mathrm{T}$ dwarfs also show considerable scatter (Fig. 3). This is an intriguing result, because the $Z$ and $J$ bands are the most transparent windows into these atmospheres, with the $Z$ band being the clearer of the two. As such, these bands are particularly sensitive to any deep variations in opacity between atmospheres, such as might be related to the upper reaches of any remaining deep silicate cloud (see Fig. 7 of Ackerman \& Marley 2001). The variation may thus be due to differences in the process(es) responsible for the removal of condensates at the $\mathrm{L}-\mathrm{T}$ transition. Since the $Z$ band is sensitive to the far wings of the optical Na-D and K I resonance lines (Burrows \& Volobuyev 2003) these variations might arise from differences in the removal of gaseous $\mathrm{Na}$ and $\mathrm{K}$, due to gravity or metallicity effects. More detailed modeling of the $\mathrm{L}-\mathrm{T}$ dwarf transition and the removal of atmospheric condensates is required to account for these observations.

\subsection{Families of Models for Comparison with the Data}

In the remainder of this paper we compare observed colors with two varieties of models from Marley et al. (2002). In the first type of model condensate opacity is ignored, although condensation chemistry is accounted for in chemical equilibrium and molecular opacities. We term these the "cloud-free" models. In the second type the effects of condensate opacity are computed using the Ackerman \& Marley (2001) cloud model. In these "cloudy" models the efficiency of condensate sedimentation is parameterized by $f_{\text {sed. }} \cdot{ }^{16}$ When the sedimentation efficiency is high (large $f_{\text {sed }}$ ) both the optical depth and vertical extent of the cloud are small. In the extreme case of no condensate sedimentation $f_{\text {sed }}=0$.

\section{5. $J-H$ and $H-K$ Colors of L Dwarfs: Unusually Red and Unusually Blue L Dwarfs}

Figure 4 shows $J-H$ plotted against $H-K$ for the L dwarfs in the sample, where spectral subclass ranges are indicated by different symbols. Overlaid are cloudy model sequences with $\log g=5$ and $f_{\text {sed }}$ values of 3 and 5. The $f_{\text {sed }}=3$ models match the data well, although a shift in modeled $J-H$ color of about 0.15 mag would encompass many more of the data points. The discrepancy is likely attributable to the modeled $\mathrm{TiO}$ bands at $J$ being too deep, causing the $J$-band magnitudes to be too faint and the $J-H$ model color to be too red. Whether this is a shortcoming in the chemical equilibrium calculation or the molecular opacities themselves is as yet unclear. $\log g=4$ models make the $H-K$ colors bluer, in better agreement with the data, but this is an unlikely gravity for these field dwarfs. (Burrows et al. 1997 show that if $T_{\text {eff }}=1500-2200 \mathrm{~K}$ and age $=1-5$ Gyr then $\log g \geq 5.0$.)

The detailed distribution of most of the data points in this color-color space is challenging to interpret as both the models and the observations show that $J-H$ and $H-K$ first become redder and then bluer with falling $T_{\text {eff. }}$. Thus, the colors double back on themselves. Despite the scattered distribution, some extreme objects stand out. The L7.5 dwarf 2MASS J2244+ 2043 and the L5.5 dwarf SDSS J0107+0041 are quite red in both colors. This may imply that their condensate cloud decks are more optically thick than average, which could arise from either less efficient sedimentation $\left(f_{\text {sed }} \sim 2\right)$ or higher metallicity.

Our sample also includes four late-type L dwarfs-SDSS J0805+4812, SDSS J0931+0327, SDSS J1104+5548, and SDSS J1331-0116 - that are unusually blue for their spectral types. As shown in Figure 3, they are bluer than average at $J-H$ by about $0.2 \mathrm{mag}$ and at $H-K$ by about $0.1 \mathrm{mag}$. Applying the shift to the models described above of about -0.15 in $J-H$, Figure 4 suggests that these dwarfs are better described by the $f_{\text {sed }}=5$ models, i.e., the sedimentation efficiency is high and the cloud optical depth is small. The spectral indices for all four of these objects show a large range - the $H$-band indices imply a late type of L9 to T1, while the $K$-band index gives an earlier type of L5.5-7.5 (the $J$-band index only implies a type earlier than T0). Our spectra show that they have enhanced $\mathrm{FeH}, \mathrm{K}$ I and $\mathrm{H}_{2} \mathrm{O}$ absorption (although the spectrum of SDSS J1104+5548 is noisy). Figure 5 shows the $J$-band spectra for SDSS J0805+4812, SDSS J0931+0327, and SDSS J1331-0116 bracketed by more typical L5.5 and L9 dwarfs. Gorlova et al. (2003) and McLean et al. (2003) show that the equivalent widths of the $J$-band $\mathrm{FeH}$ and $\mathrm{K}$ I features peak at spectral types around L3 and then become smaller as the $\mathrm{Fe}$ condenses into grains and $\mathrm{K}$ is lost to $\mathrm{KCl}$. The strengths of these features in SDSS J0805+4812, SDSS J0931+0327, and SDSS J1331-0116 are similar to those of the early $\mathrm{L}$ types, while their $\mathrm{H}_{2} \mathrm{O}$ bands are more typical of the latest $\mathrm{L}$ dwarfs, supporting the interpretation that we are looking deep into unusually condensate-free atmospheres. The possibility of low metallicity should also be

\footnotetext{
16 Ackerman \& Marley (2001) employed the parameter " $f$ rain" to describe the efficiency of condensate sedimentation in a brown dwarf atmosphere. Strictly speaking, "rain" is falling water, and this term has now been replaced by $f_{\text {sed. }}$.
} 


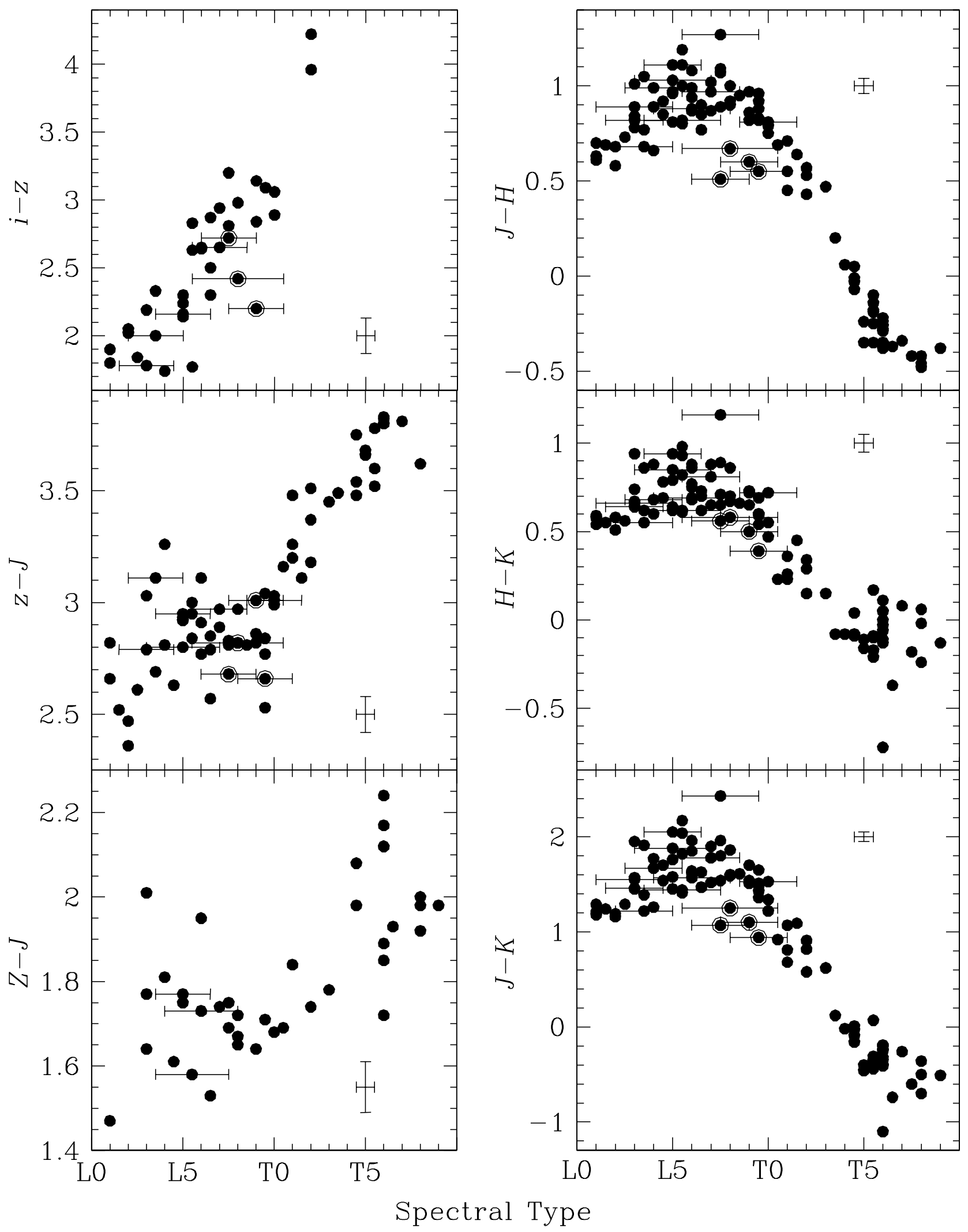

Fig. 3. - Colors as a function of spectral type. Note that SDSS $i$ and $z$ measurements are on the AB system, while the other magnitudes are on the Vega $=0$ system. $Z$ is on the UKIRT (UFTI) photometric system, while $J H K$ are on the MKO-NIR system. Points of low signal-to-noise ratio are not plotted. Typical error bars are shown, as well as those for dwarfs with spectral type uncertainty larger than one subclass. Data points for the four unusually blue late L dwarfs are ringed $(\S 5.5)$. 


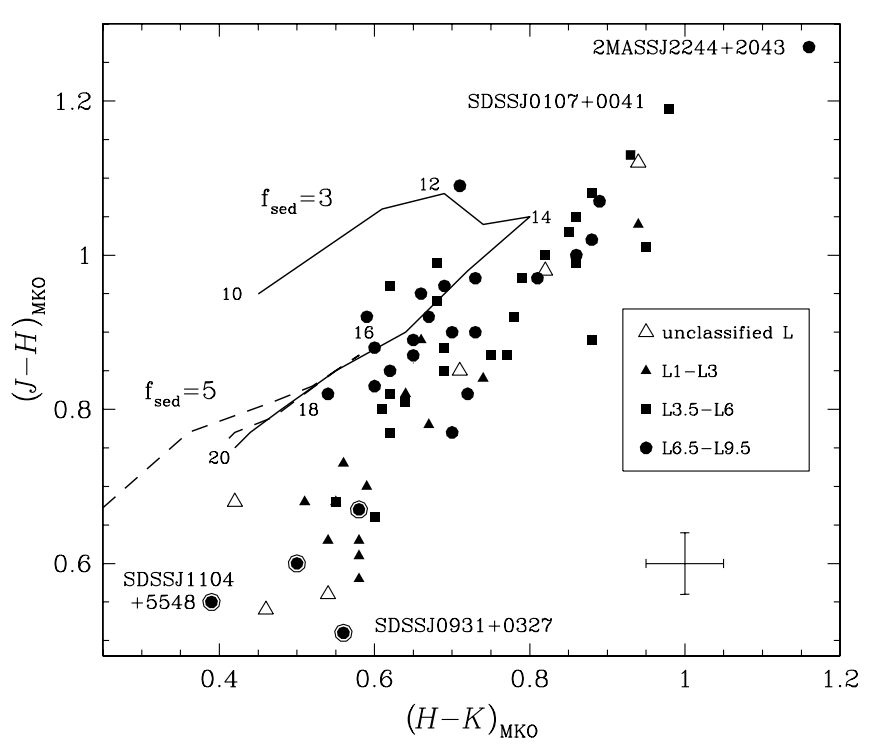

FIg. 4. $-J-H$ vs. $H-K$ for the $\mathrm{L}$ dwarfs in Table 9. Model sequences (Marley et al. 2002) are shown for $f_{\text {sed }}=3$ (solid line) and $f_{\text {sed }}=5$ (dashed line), $\log g=5.0$, cloudy atmospheres. $T_{\text {eff }}$ is indicated in units of $100 \mathrm{~K}$ along the $f_{\text {sed }}=3$ sequence. Dwarfs with extreme colors are identified, data points for four unusually blue late L dwarfs are ringed. A typical error bar is shown.

considered. Burgasser et al. (2003a) identified a late L dwarf (2MASS J05325346+8246465) whose extremely blue nearinfrared colors are similar to those of the mid T types. This high-velocity dwarf appears to be an extremely metal-poor halo subdwarf with strong $\mathrm{FeH}$ features as well as $\mathrm{H}_{2}$ absorption that depresses the $H$ and $K$ band fluxes. Cruz et al. (2003) identify two early L dwarfs (2MASS J1300425+ 191235 and 2MASS J172139+334415) that are bluer than

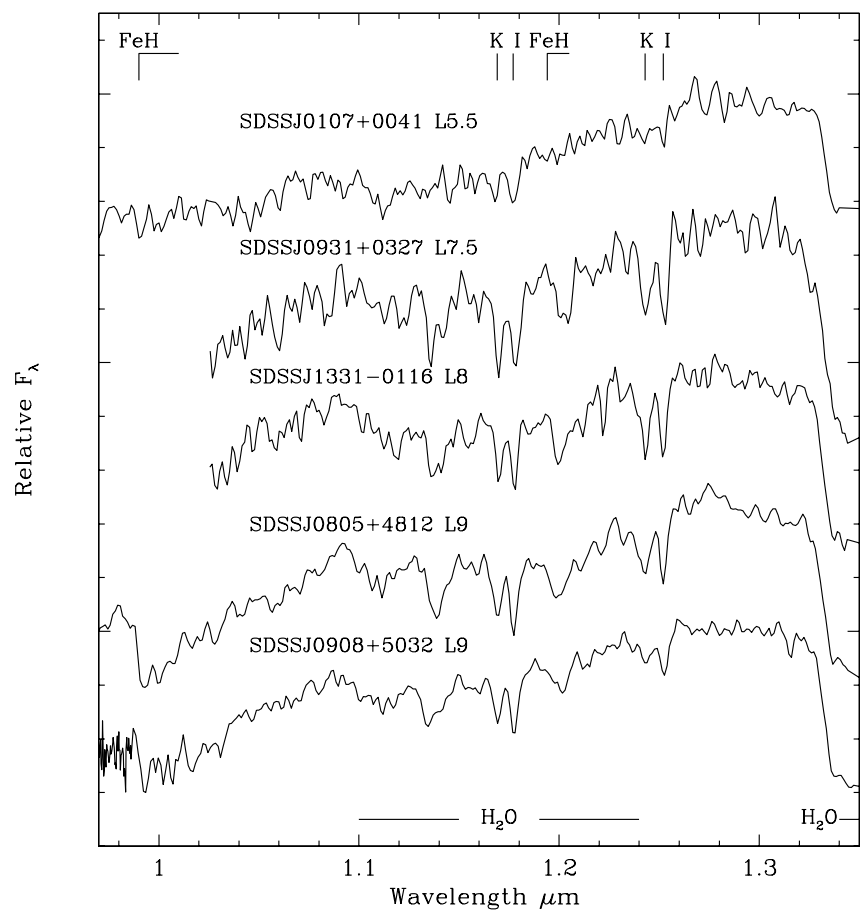

FIG. 5.- $J$-band spectra $(R \approx 600)$ for three of the four unusually blue late L dwarfs, bracketed by more typical L5.5 and L9 dwarfs. We identify the strong FeH features at 0.99 and $1.19 \mu \mathrm{m}, \mathrm{K}$ I lines at $1.169,1.177,1.243$, and $1.252 \mu \mathrm{m}$, and $\mathrm{H}_{2} \mathrm{O}$ bands around 1.15 and $1.33 \mu \mathrm{m}$. See Cushing et al. (2003) for a more complete identification of $\mathrm{FeH}$ features.

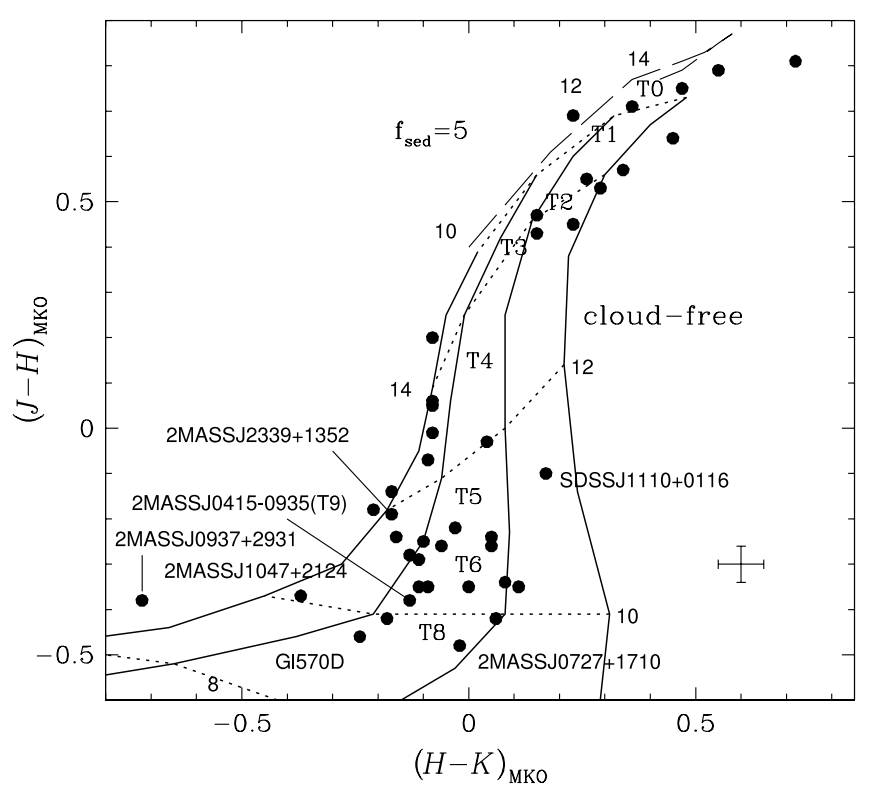

FIg. 6. $-J-H$ vs. $H-K$ for the T dwarfs in Table 9. Model sequences from Marley et al. (2002) are shown for $f_{\text {sed }}=5$ and $\log g=5.0$ cloudy models (dashed line) and cloud-free models (solid line), where from left to right $\log g=5.5,5.0,4.5$, and 4.0. $T_{\text {eff }}$ is indicated in units of $100 \mathrm{~K}$ and for the cloud-free models dotted lines indicate constant $T_{\text {eff. }}$ The typical error is shown at the bottom right, and the sequence of spectral type is shown. Dwarfs of particular interest are labelled (see $\S 5.6$ ). For field T dwarfs, gravity correlates with mass, and the range in $\log g$ shown here translates into masses 75 , 35,15 , and $5 M_{\text {Jupiter }}$ from left to right.

average at $J-K$ by about $0.2 \mathrm{mag}$. As condensate clouds are optically thin for early L dwarfs, and these dwarfs have significant proper motion, they may also be part of a lowmetallicity population.

\section{6. $J-H$ and $H-K$ Colors of T Dwarfs: Gravity and Mass Determinations}

The $H-K$ and $J-K$ colors of T5-T9 dwarfs are scattered (Fig. 3), even though the $H$ - and $K$-band indices of G02 yield consistent classifications. Figure 6 shows $J-H$ against $H-K$ for the $\mathrm{T}$ dwarfs in the sample with sequences from the cloudfree and cloudy $f_{\text {sed }}=5$ models by Marley et al. (2002) overlaid. The synthetic $H-K$ colors for the late $\mathrm{T}$ dwarfs and the range in color over a plausible range of gravities of $\log g=4.5-5.5$ reproduce the observed colors extremely well.

Gl 570 D provides a further test for the model-data correspondence shown in Figure 6. Geballe et al. (2001) fitted models to the absolute luminosity of G1 570D and used age constraints to find $T_{\text {eff }}=784-824 \mathrm{~K}$ and a surface gravity in the range 5.00-5.27. This gravity range is consistent with that implied by Figure 6 . The effective temperature implied by Figure 6 , however, is high by about $150 \mathrm{~K}$. Although Geballe et al. (2001) found that their best-fitting models generally reproduced the $J H K$ spectrum of Gl 570D quite well, there were notable discrepancies. In particular the notorious inadequacy of the $H$-band methane opacity database and the tendency of all clear-atmosphere models to overestimate the water-band depths limit the fidelity of the fit. These deficiencies both result in the best-fitting temperature contours in Figure 6 being somewhat too warm.

Further, the trends shown in Figure 6 may break down for lower temperatures. One interesting challenge is the T9 dwarf 2MASS J0415-0935, the latest and coolest T dwarf currently known, with $T_{\text {eff }} \approx 700 \mathrm{~K}$ (Golimowski et al. 2004; Vrba et al. 
TABLE 11

Surface Gravities of T5 and Later Dwarfs Estimated from $H-K$ Color

\begin{tabular}{lll}
\hline \hline \multicolumn{1}{c}{$\log g \approx 4.5$} & \multicolumn{1}{c}{$\log g \approx 5.0$} & \multicolumn{1}{c}{$\log g \approx 5.5$} \\
\hline 2MASS J0243-2453 & 2MASS J0415-0935 & 2MASS J0755+2212 \\
2MASS J0727+1710 & 2MASS J1225-2739AB & 2MASS J0937+2931 \\
2MASS J1217-0311 & 2MASS J1503+2525 & 2MASS J1047+2124 \\
G1 229B & 2MASS J1553+1532AB & 2MASS J1534-2952AB \\
SDSS J1110+0116 & 2MASS J2356-1553 & 2MASS J2339+1352 \\
SDSS J1346-0031 & G1 570D & SDSS J0830+0128 \\
SDSS J1758+4633 & SDSS J0741+2351 & $\ldots$ \\
SDSS J2124+0100 & SDSS J0742+2055 & $\ldots$ \\
$\ldots$ & SDSS J1231+0847 & $\ldots$ \\
$\ldots$ & SDSS J1624+0029 & $\ldots$ \\
\hline
\end{tabular}

2004). Figures 6,8 , and 9 show that instead of being bluer in $J-H, H-K$, and $J-K$ than Gl $570 \mathrm{D}$, it is redder, by $0.2 \mathrm{mag}$, in $J-K$. While models by Marley et al. (2002) and Burrows et al. (2003) predict that, indeed, the coolest dwarfs will become redder in $J-K$ with falling $T_{\text {eff }}$ and the onset of water cloud formation, this happens only for models with $T_{\text {eff }} \lesssim 500 \mathrm{~K}$, unless the brown dwarf is older than $7 \mathrm{Gyr}$ and more massive than $40 M_{\text {Jupiter }}$. The condensation of the alkalis into their solid chloride forms may also lead to redder colors at these kinds of temperatures (Lodders 1999; Marley 2000; Burrows et al. 2003).

Despite these discrepancies the overall trends seen in Figure 6 can be understood in the context of our presently limited understanding of brown dwarf atmospheres. At the effective temperatures of late $\mathrm{T}$ dwarfs, the $K$-band flux is very sensitive to gravity. This is because the opacity of pressureinduced $\mathrm{H}_{2}$ absorption is proportional to the square of the local gas number density. Higher gravity objects of a given $T_{\text {eff }}$ tend to be cooler at a given pressure than lower gravity dwarfs and thus have denser, more opaque atmospheres. Hence, highgravity objects tend to be dimmer at $K$ and bluer in $H-K$ than comparable lower gravity objects. 2MASS J0937+2931 shows a particularly depressed $K$-band flux standing out in Figure 6, presumably because of strong $\mathrm{H}_{2}$ opacity (Burgasser et al. 2002a). Structural models imply an upper limit to $\log g$ of 5.5 for brown dwarfs (e.g., Burrows et al. 1997), and therefore Figure 6 suggests that 2MASS J0937+2931 may be both a high-gravity and a low-metallicity dwarf, as also suggested by Burgasser et al. (2003b). While $\mathrm{H}_{2}$ opacity is also enhanced by decreasing metallicity (e.g., Saumon et al. 1994; Borysow et al. 1997), variations in metallicity are less likely than variations in gravity for this sample of local field brown dwarfs.

$H-K$ appears to be an easy-to-obtain and straightforward indicator of gravity for late $\mathrm{T}$ dwarfs. This is significant as, for a field sample with a likely range in age of $1-5 \mathrm{Gyr}$ (Dahn et al. 2002 estimate 2-4 Gyr based on kinematic arguments), gravity corresponds directly to mass. This tight relationship is due to the small dependence of radius on age or mass for brown dwarfs older than about $200 \mathrm{Myr}$ (Burrows et al. 2001). Figure 9 of Burrows et al. (1997) shows that $\log g=4.5$ implies a mass of $15 M_{\text {Jupiter }}, \log g=5.0$ a mass of $35 M_{\text {Jupiter }}$, and $\log g=5.5$ a mass of $75 M_{\text {Jupiter }}$. We list the $H-K$ implied surface gravities for the later T dwarfs in Table 11.

Recent investigations of spectroscopic gravity indicators for $\mathrm{L}$ and $\mathrm{T}$ dwarfs include those by Lucas et al. (2001), Burgasser et al. (2003b), Gorlova et al. (2003), Martín \& Zapatero Osorio (2003), and McGovern et al. (2004). Figure 2 of Martín \& Zapatero Osorio shows synthetic spectra for $T_{\text {eff }}=1000 \mathrm{~K}$ from COND models by Allard et al. (2001). The models imply that the lines of $\mathrm{K}$ I at 1.243 and $1.254 \mu \mathrm{m}$ become weaker with increasing gravity for $\mathrm{T}$ dwarfs. ${ }^{17}$ Figure 7 shows $J$-band spectra of three T6 $( \pm 0.5)$ dwarfs (SDSS J1110+0116, 2MASS J2339+13, and 2MASS J0937+ 2931) and two T8 dwarfs (2MASS J0727+1710 and Gl 570D). These dwarfs span a range in $H-K$ color and are identified in Figure 6. It can be seen that as $H-K$ increases from top to bottom in Figure 7, the $\mathrm{K}$ I lines strengthen, supporting the interpretation of increasing $H-K$ as being due to decreasing gravity. The increase in $\mathrm{K}$ I equivalent widths for these dwarfs is confirmed by the higher resolution NIRSPEC data of McLean et al. (2003).

Comparison of our Figure 7 with Figure 2 of Martín \& Zapatero Osorio (2003) shows that the depths of the $\mathrm{K}_{\mathrm{I}}$ lines seen in 2MASS J0937+2931 are similar to the model predictions for $\log g \sim 5.5$ and that the lines seen in SDSS $\mathrm{J} 1110+0116$ are almost as strong as the synthetic spectrum with $\log g \sim 3.5$. Figure 6 and the models of Marley et al. (2002) imply that SDSS J1110+0116 has $\log g$ between 4.0 and 4.5. There is as yet no direct measurement of the effective temperature of this dwarf, but the effective temperatures of other T6 dwarfs are in the range 900-1075 K (Golimowski et al. 2004). If SDSS J1110+0116 has $T_{\text {eff }} \approx 1000 \mathrm{~K}$ and $\log g \approx$ 4.2, the evolutionary models of Burrows et al. (2003, their Fig. 1) imply that it is a $10 M_{\text {Jupiter }}$ brown dwarf with an age of about $1 \times 10^{8} \mathrm{yr}$, i.e., similar to that of the Pleiades cluster. However, we argue in $\S 5.7 .4$ that one might shift the model contours on Figure 6 up and to the right to bring them into better agreement with observed $J-K$ and measured effective temperatures. In that case SDSS J1110+0116 would have a somewhat larger gravity, mass, and age. We thus adopt a more conservative estimated mass of $10-15 M_{\text {Jupiter }}$ and an age of $1-3 \times 10^{8}$ yr. We note also that the candidate young-cluster

\footnotetext{
${ }^{17}$ Theoretically this is explained by the fact that the column abundance of molecules above a given pressure level $P$ in an atmosphere is proportional to $P / g$, where $g$ is the gravity. In a higher gravity atmosphere an outside observer must, all else being equal, look to higher pressure to observe the same column of absorber as in a lower gravity atmosphere. The calculations of Lodders (1999) show that with rising pressure at a fixed temperature chemical equilibrium increasingly favors $\mathrm{KCl}$ over $\mathrm{K}$. Thus in a higher gravity atmosphere the total column of potassium above the floor set by the continuum opacity is less than in a lower gravity model. The sensitivity to gravity should be greater in later $\mathrm{T}$ (lower $T_{\text {eff }}$ ) atmospheres since the line forming region (roughly 1000 and $1500 \mathrm{~K}$ in the 1.15 and $1.25 \mu \mathrm{m}$ regions, respectively) falls closer to the chemical equilibrium boundary than for earlier T dwarfs (see Fig. 2 of Lodders 1999). In addition higher pressures produce greater line broadening, thus decreasing the line depth.
} 


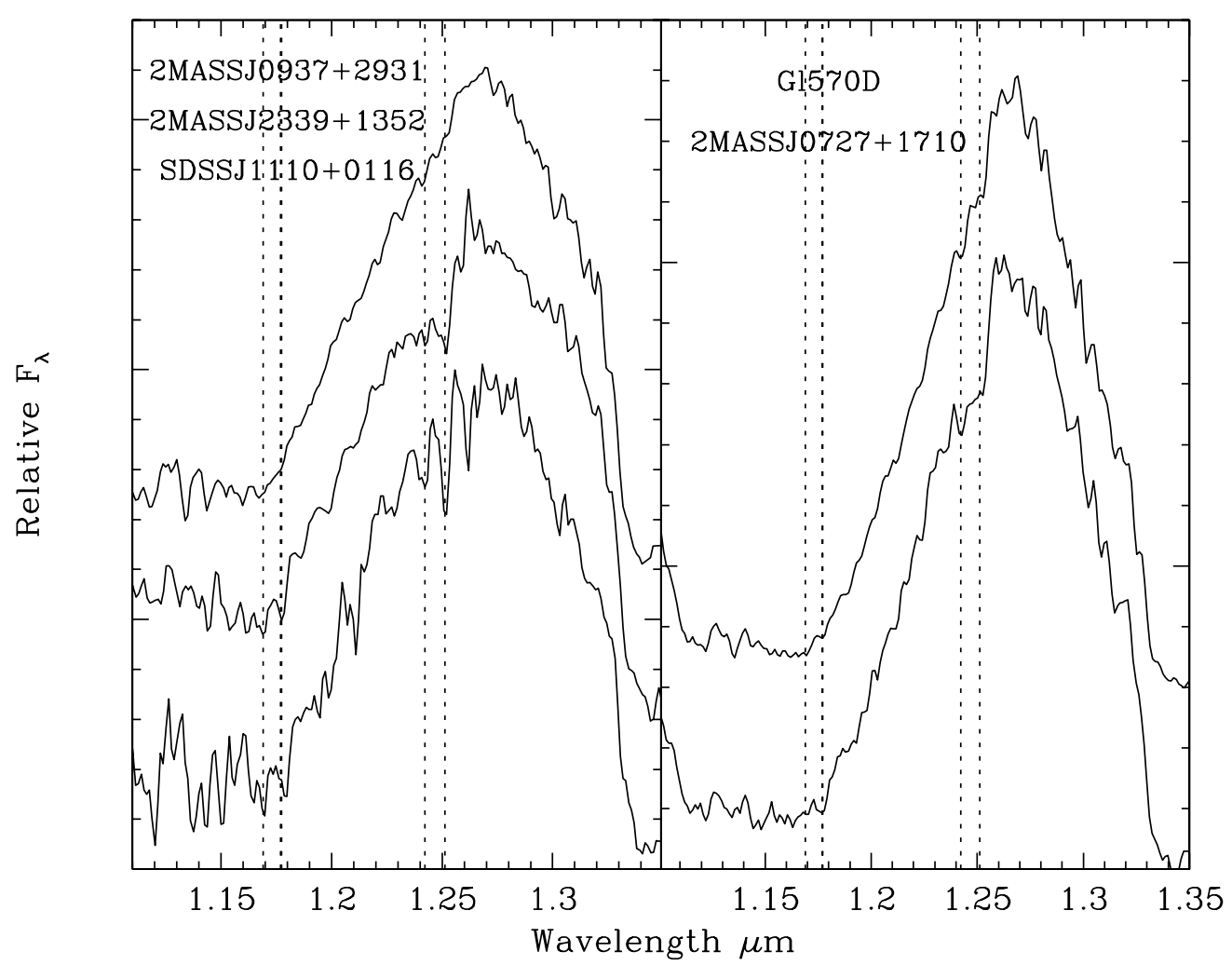

FIG. 7.-Normalized $J$-band spectra $(R \approx 600)$ for a sample of T6 $( \pm 0.5)$ dwarfs (left $)$ and T8 dwarfs $($ right $)$. The location of $\mathrm{K}_{\mathrm{I}}$ doublets are shown by the dotted lines. The dwarfs have $H-K$ increasing from top to bottom, and the $\mathrm{K}$ I features strengthen from top to bottom, supporting the conclusion that log $g$ decreases from top to bottom.

T dwarf S Ori 70 has an unusually red $H-K_{\mathrm{s}}$ color (Zapatero Osorio et al. 2002), apparently consistent with $\log g<4$.

\subsection{Absolute Magnitudes: The L-T Transition}

It has been apparent since the earliest discoveries of $\mathrm{L}$ and $\mathrm{T}$ dwarfs that L-type objects evolve into T-type objects as they cool. With decreasing effective temperatures the condensation level for the principle L dwarf condensates (iron and silicates) falls progressively deeper in the atmosphere. Unless upward mixing is very efficient, the clouds will eventually disappear beneath the photosphere, whose location is strongly wavelength dependent for these objects. Both the general evolutionary cooling trend and the removal of condensates produce lower atmospheric temperatures; under these conditions the equilibrium chemistry rapidly begins to favor $\mathrm{CH}_{4}$ over $\mathrm{CO}$ as the dominant $\mathrm{C}$-bearing species. With less photospheric condensate to veil the molecular bands and the growing importance of $\mathrm{CH}_{4}$ opacity in the $\mathrm{K}$ band, the objects turn to the blue in $J-K$. Marley (2000) employed a simple, one scale-height thick cloud layer to demonstrate that the sinking of a finite cloud deck explains the red to blue transition in $J-K$. This was subsequently confirmed by models employing more elaborate cloud models (Marley et al. 2002; Tsuji 2002; Allard et al. 2003).

The absolute magnitudes presented here can be used to better understand this behavior. Figures 8 and 9 show, respectively, absolute $J$ and $K$ magnitude against spectral type and $J-K$ color. A fifth-order polynomial fit is shown to absolute magnitude against spectral type and the coefficients of the fit are given in Table 12. Known binaries were removed from the sample before fitting the data; the mean scatter around the fit is $0.4 \mathrm{mag}$ for $M_{J}$ and $0.3 \mathrm{mag}$ for $M_{K}$.
As noted by Burgasser et al. (2002b), these data suggest that the $\mathrm{L}-\mathrm{T}$ dwarf transition may be more complex than implied by the picture of a continuously sinking cloud. The most notable discrepancy between the simple picture and the data shown in these figures is the brightening seen at $J$-band as the objects transition from $L$ to $T$. In this section we summarize various suggested mechanisms to explain this behavior and compare their predictions to the photometric data presented here.

\subsubsection{Thin Cloud Decks}

A finite-thickness cloud deck forming progressively lower in the atmosphere will eventually disappear from sight. In the opposite extreme dust that is well mixed through the entire observable atmosphere, as in the DUSTY models of the Lyon group (e.g., Allard et al. 2001), will by definition never disappear. Models of such objects show that they simply become progressively redder as they cool and thus, because of veiling of the changing molecular bands, never exhibit an $\mathrm{L}-\mathrm{T}$ transition.

Although models with finite-thickness cloud decks do move from red, L-like colors to blue, T-like colors, they tend to do so relatively slowly. This is because the cloud always has a finite thickness and thus does not disappear from a given bandpass instantaneously. During the time the cloud is departing, say, from $J$-band visibility, the overall atmosphere is continuing to cool and become fainter. Thus, in a color-magnitude diagram (Figs. 8 and 9) models with finite-thickness clouds that are opaque enough to reach the colors of the latest $\mathrm{L}$ dwarfs $(J-K \sim 2)$ tend to leisurely turn to the blue as they cool and so reach the colors of the early $\mathrm{T}$ dwarfs at too faint magnitudes. The $f_{\text {sed }}=3$ model in Figure 8 is an example. 


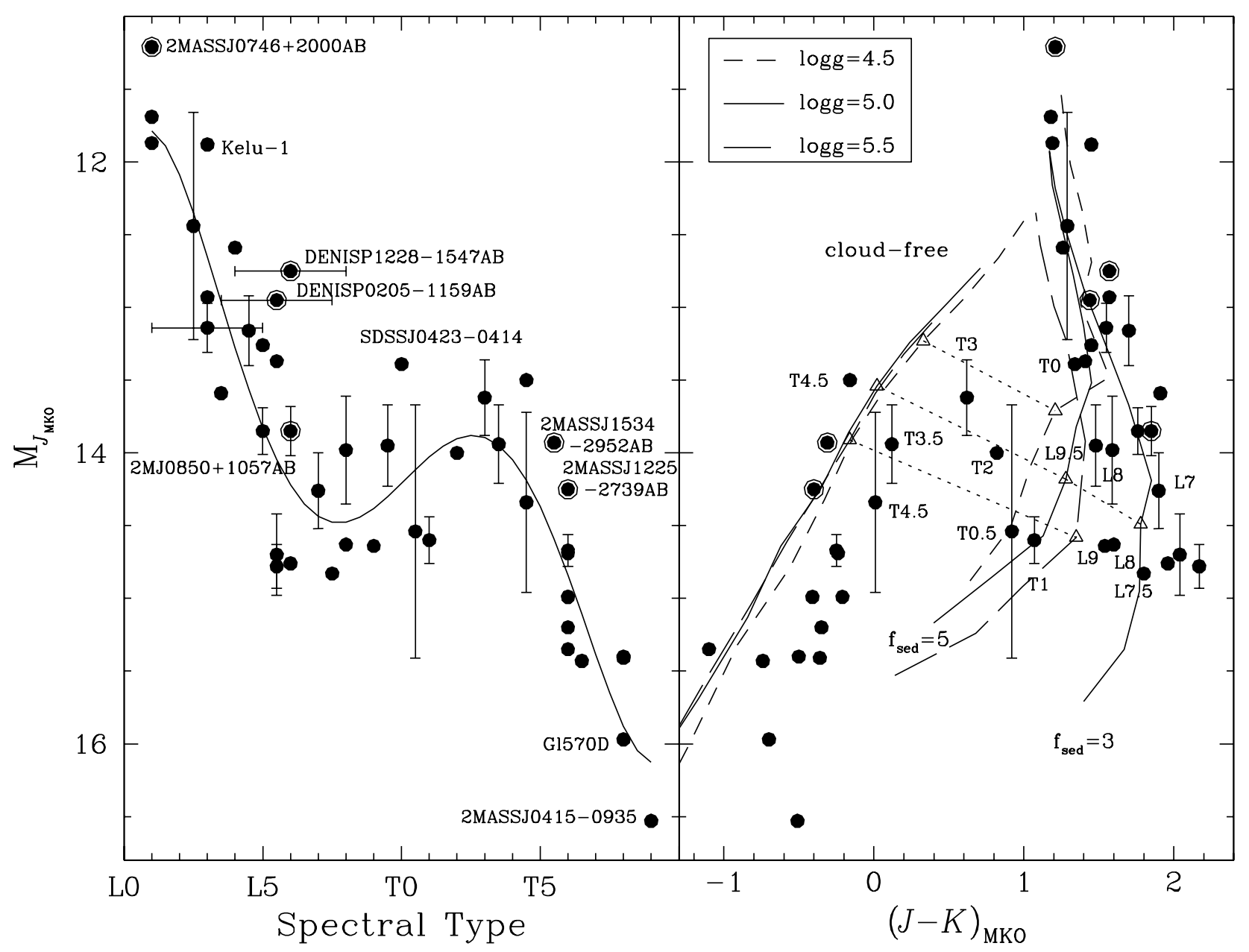

FIG. 8. $-M_{J}$ as a function of spectral type and $J-K$ color. Error bars are shown where the distance modulus is uncertain by $\geq 0.1$ mag and where type is uncertain by greater than 1 subclass. Known binaries are indicated by ringed symbols. In the left panel the known binaries and other apparently superluminous dwarfs are labelled, as well as the two faintest dwarfs. A fifth-order polynomial fit to $M_{j}$ : type is shown (coefficients are given in Table 12). In the right panel all objects with types between L7 and T4.5 are identified. Model $M_{J}$ against $J-K$ sequences are shown for $f_{\text {sed }}=3, f_{\text {sed }}=5$, and cloud-free models from Marley et al. (2002). The dotted lines between the triangles connect the $T_{\text {eff }}=1300 \mathrm{~K}$ points on each model sequence (see discussion in $\S 5.7$ ).

Tsuji \& Nakajima (2003) proposed an interesting solution to this problem. They found a family of models with relatively thin cloud decks in which the turnoff from red to blue in $J-K$ was a function of gravity. In these models low-gravity $10 M_{\text {Jupiter }}$ objects depart from what might be called the "Ltype cooling sequence" (the progressive reddening in $J-K$ with later spectral type) and turn from red to blue at a point almost 2 mag brighter than high-gravity $70 M_{\text {Jupiter }}$ objects. A similar, though less extreme, bright turn off is seen in the $f_{\text {sed }}=5$ family of models in our Figures 8 and 9 . Tsuji \& Nakajima then suggest that there is not a single evolutionary path in which objects first fade at $J$ band as they get redder and then brighten as they turn blue. Rather, they propose that the brighter transition $\mathrm{T}$ dwarfs are low-mass objects that cooled to mid-L type and then turned from red to blue colors around $M_{J} \sim 13.3$. Dimmer transition objects would represent intermediate-mass brown dwarfs that turned off the $\mathrm{L}$ cooling sequence at a later $\mathrm{L}$ type and redder $J-K$, and the latest L's would represent the highest-mass objects.

This model makes a number of interesting predictions. First, the $M_{J}$ versus $J-K$ phase space between the $\mathrm{L}$ and $\mathrm{T}$ dwarfs should eventually be found to be fairly evenly populated both at brighter and fainter magnitudes than is shown by the transition objects detected to date. Second, bright early T dwarfs, like SDSS J1021-0304 (T3) or 2MASS J0559-1404 (T4.5), should be fairly low mass objects, while the latest and reddest L dwarfs, like 2MASS J1632+1904 (L7.5), should be fairly high mass objects. Emerging gravity indicators should be able to test this hypothesis.

\subsubsection{Patchy Clouds}

Plotted in the right panels of Figures 8 and 9 are model sequences from Marley et al. (2002). Both cloud-free and cloudy models are shown, the latter with sedimentation parameters $f_{\text {sed }}=3$ and 5 . For the $f_{\text {sed }}=5$ and the no-cloud models, three gravities are shown $(\log g=4.5,5$, and 5.5). Only $\log g=5$ is shown for $f_{\text {sed }}=3$. Model effective temperatures are given on the right axis of Figure 9. The general agreement between the observed $\mathrm{L}$ colors and the $f_{\text {sed }}=3$ models seen in Figures 4, 8, and 9, suggests that the JHK colors of the L dwarfs can be explained by a uniform global cloud model. The cloud's vertical extent and optical depth are limited by sedimentation. Models with much less or much more efficient sedimentation would generally be too red or too blue, respectively, than most of the L dwarf population. However, the existence of a few dwarfs that are redder and a few that are bluer than most ( $§ 5.5$ ) implies that about $10 \%$ of the $\mathrm{L}$ dwarf population would require more 


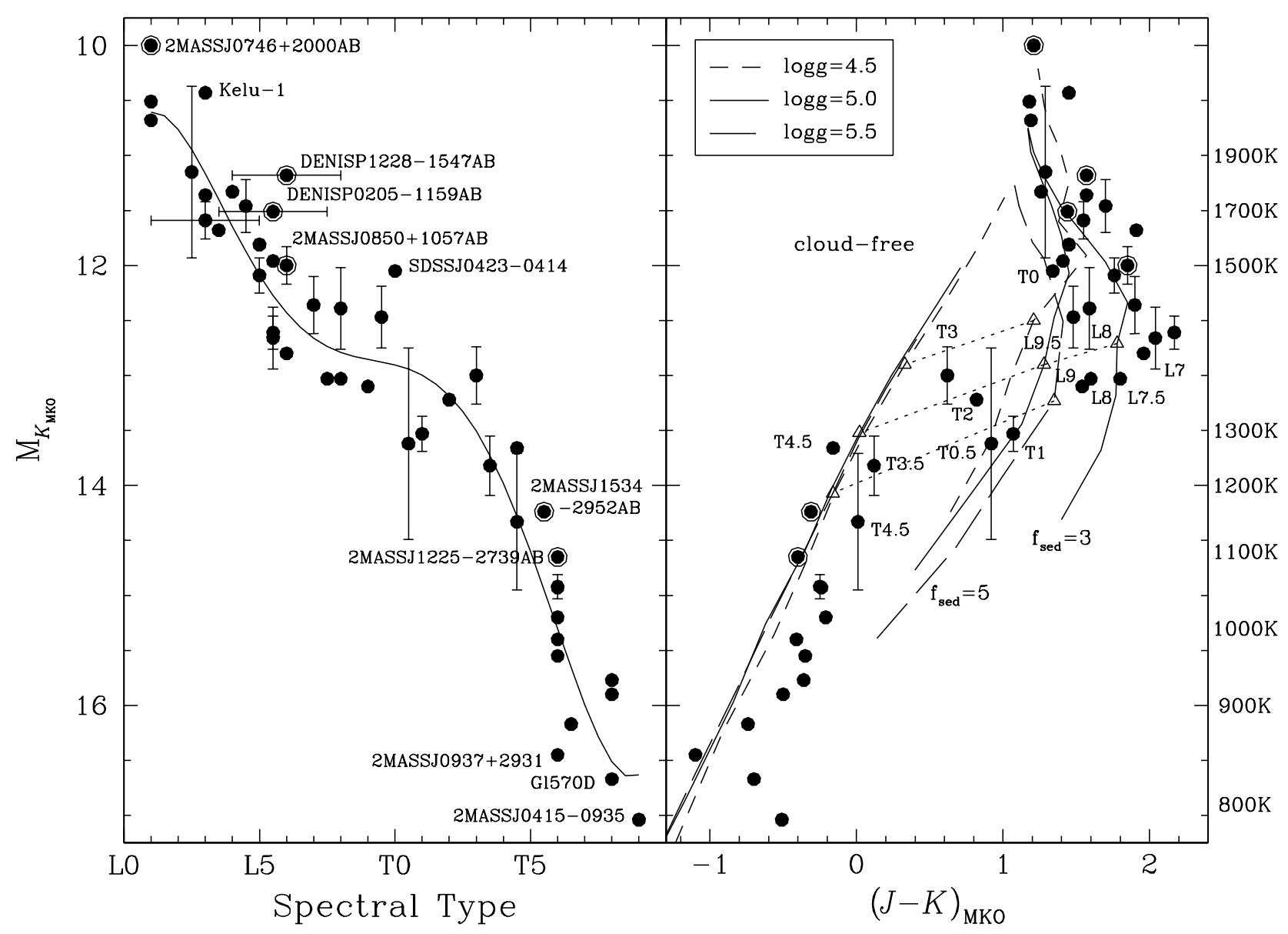

FIG. 9. $-M_{K}$ as a function of spectral type and $J-K$ color. Error bars are shown where the distance modulus is uncertain by $\geq 0.1$ mag and where type is uncertain by greater than 1 subclass. Known binaries are indicated by ringed symbols. In the left panel the known binaries and other apparently superluminous dwarfs are labelled as well as the three faintest dwarfs. A fifth-order polynomial fit to $M_{K}$ : type is shown (coefficients are given in Table 12). In the right panel all objects with types between L7 and T4.5 are identified. Model $M_{K}$ against $J-K$ sequences are shown for $f_{\text {sed }}=3, f_{\text {sed }}=5$, and cloud-free models from Marley et al. (2002). The dotted lines between the triangles connect the $T_{\text {eff }}=1300 \mathrm{~K}$ points on each model sequence. Effective temperatures from the log $g=5.0$ models are shown on the right axis, where $T_{\text {eff }}=1500-1900 \mathrm{~K}$ correspond to the $f_{\text {sed }}=3$ models and $T_{\text {eff }}=800-1300 \mathrm{~K}$ correspond to the cloud-free models

extreme models. These variations could arise from either metallicity or sedimentation efficiency differences between objects.

However, the $f_{\text {sed }}=3$ models turn too slowly to the blue and reach the colors of the bluest $\mathrm{T}$ dwarfs at $J$-band magnitudes that are too faint. Faced with this difficulty of finite cloud layers taking too long to disappear in models like those of Tsuji \& Nakajima (2003), Burgasser et al. (2002b) propose a different mechanism for the $\mathrm{L}-\mathrm{T}$ transition. Drawing on a suggestion from Ackerman \& Marley (2001), Burgasser et al. (2002b) propose that the L-T transition region is marked by the appearance of holes in the global cloud deck, not unlike those seen in the " $5 \mu \mathrm{m}$ hot spots" on Jupiter. Deeply seated flux, particularly in the clear $Z$ and $J$ windows, would then pour out of these holes, pushing the disk-integrated color to the blue. Indeed, Burgasser et al. (2002b) found that a relatively small fraction of holes would appreciably move a late L-type object toward the blue in $J-K$. They argued that such a mechanism would explain the brightening observed at $Z$ and $J$, and not at other bands, across the transition, and also the observed resurgence in $\mathrm{FeH}$ absorption from the latest Ls to the early to mid T's.

To illustrate the effect such holes might have, we have joined with a dotted line the magnitude-color values for the cloudy and cloud-free models at $T_{\text {eff }}=1300 \mathrm{~K}$ in both Figures 8 and $9 .{ }^{18}$ The agreement between the data points for T1 to T3 dwarfs and the cloudy to cloud-free interpolations in Figures 8 and 9 is generally good. The parameters $T_{\text {eff }}=1300 \mathrm{~K}$ and $\log g=$ 4.5-5.5 appear to bracket the known transition objects in these plots, in fair agreement with Golimowski et al. (2004), who show that there is an apparent plateau at $T_{\text {eff }} \approx 1450 \mathrm{~K}$ for types L7 to T4. The cloud-clearing model simply posits that at a given effective temperature the global cloud deck begins to break up, perhaps because it has settled sufficiently deeply into the convection zone that it becomes subject to the global circulation pattern. The observed constancy of $T_{\text {eff }}$ across the transition is not required by this hypothesis, although it does raise problems for the Tsuji \& Nakajima (2003) suggestion of continuous cooling across the transition.

If the clearing does happen over a narrow temperature range, with a large spectroscopic change occurring over a small change in temperature and luminosity as the brown dwarf cools, we would not expect to discover many early $\mathrm{T}$ dwarfs. However, about one-third of our T dwarf sample is

\footnotetext{
${ }^{18}$ Note that Burgasser et al. presented a slightly more sophisticated interpolation, computing colors using a weighted sum of flux produced by clear and cloudy temperature profiles. The straight lines in Figs. 8 and 9 are reasonable approximations to their "clearing models."
} 
TABLE 12

Coefficients of Polynomial Fit to $M_{j}$ :Type and $M_{K}$ :Type

\begin{tabular}{lcccccr}
\hline \hline Magnitude & $c_{0}$ & $c_{1}$ & $c_{2}$ & $c_{3}$ & $c_{4}$ & $c_{5}$ \\
\hline$M_{J_{\text {MKO }}}$ & 12.03 & $-6.278 \mathrm{e}-1$ & $4.500 \mathrm{e}-1$ & $-6.848 \mathrm{e}-2$ & $3.986 \mathrm{e}-3$ & $-7.923 \mathrm{e}-5$ \\
$M_{K_{\mathrm{MKO}}}$ & 10.93 & $-6.485 \mathrm{e}-1$ & $3.876 \mathrm{e}-1$ & $-5.819 \mathrm{e}-2$ & $3.524 \mathrm{e}-3$ & $-7.351 \mathrm{e}-5$ \\
\hline
\end{tabular}

Note.-Fit is valid for L1 to T9 and is applied as mag $=c_{0}+c_{1} \times$ Type $+c_{2} \times$ Type $^{2}+c_{3} \times$ Type $^{3}+$ $c_{4} \times$ Type $^{4}+c_{5} \times$ Type $^{5}$, where type is an integer such that $01=\mathrm{L} 1,10=\mathrm{T} 0,19=\mathrm{T} 9$.

made up of types $\mathrm{T} 0-\mathrm{T} 3.5$, with a possible dearth of $\mathrm{T} 3-4$ types (Fig. 3). A study of the spectral type distribution in an SDSS magnitude-limited sample will be the subject of a future paper (Collinge et al. 2002).

\subsubsection{Sudden Downpour}

Rather than relying on spatial inhomogeneities, Figures 8 and 9 suggest a third possibility for the $\mathrm{L}-\mathrm{T}$ transition, which we term the "sudden downpour" model. The $f_{\text {sed }}=3$ models do a reasonably good job of reproducing the colors of the latest L dwarfs. Like the thin Tsuji \& Nakajima (2003) cloud, models with more efficient sedimentation (larger $f_{\text {sed }}$ ) turn off the L dwarf cooling track sooner (at brighter magnitudes) than seems to be consistent with the available data. However, one might argue that $\mathrm{L}$ dwarfs first cool at essentially constant $f_{\mathrm{sed}}$, then at around $T_{\text {eff }}=1300 \mathrm{~K} f_{\text {sed }}$ begins to gradually increase from $\sim 3$ to infinity at roughly fixed effective temperature. This rapid increase in the efficiency of sedimentation would, in essence, produce a torrential rain of condensed iron and silicate grains. Unlike the Tsuji \& Nakajima (2003) mechanism, this would begin at the late L spectral type for all masses. T1 to T4 dwarfs would represent different stages of this cloud thinning process. Figures 8 and 9 show where the $f_{\text {sed }}=5$ models would lie, for example. Once grains are essentially completely removed from the atmosphere, the object would continue to evolve and cool.

A useful diagnostic for evaluating the various transition models may be gravity. The sudden downpour mechanism, for example, would predict that the T3.5 dwarf SDSS J1750+1759 (see Fig. 8) would have $\log g \sim 5$.4. Evolution tracks for the patchy cloud model curve downward at blue $J-K$ compared with the straight lines shown in the figure, and thus this model would predict a smaller gravity, say, $\log g=5$. On the other hand, Tsuji \& Nakajima (2003) would predict that since this relative bright $\mathrm{T}$ dwarf has already made the transition to blue $J-K$, it must be relatively low in mass and have a substantially lower gravity, say, $\log g=4$. There are also gravity tests among the late L dwarfs, although these are more subtle since all of the models would predict that the faintest and reddest $\mathrm{L}$ dwarfs will have progressively higher masses. Tsuji \& Nakajima (2003) predict that the lowest-mass dwarfs turn to the blue relatively early. The downpour model also predicts that lower masses turn blueward earlier than higher masses, as can be seen by studying the tracks for the various gravities in the $f_{\text {sed }}=5$ case. However, this turn off happens at later spectral types than in the Tsuji \& Nakajima model and is accompanied by a subsequent brightening in $M_{J}$. For example, the L7 dwarf labeled in Figure 8 could have $\log g \geq 4.8$ under the cloud clearing and downpour models, but Tsuji \& Nakajima (2003) would predict a higher minimum gravity, likely around 5.3 or so. Certainly, there are hints in Figure 8 of a width in $M_{J}$ to the transition, and this will facilitate such tests.
There are also other diagnostics to consider. The patchycloud model straightforwardly accounts for the resurgence seen in FeH absorption across the transition (Burgasser et al. $2002 \mathrm{~b}$ ), and it is not clear that other models can account for this. Clearly, more modeling of all mechanisms must be completed to better define gravity and other diagnostics of the transition mechanism. Unfortunately, gravity indicators among the late $\mathrm{L}$ and early $\mathrm{T}$ field dwarfs are elusive, and it may be difficult to use them to definitively test the models. (One might expect that the Li test [Martín, Rebolo, \& Magazzu 1994] could be used to identify high-gravity dwarfs, since only brown dwarfs more massive than $0.06 M_{\odot}$ will have burned Li during their evolution. As a practical matter, however, the $\mathrm{Li}$ line at $0.6708 \mu \mathrm{m}$ in late $\mathrm{L}$ and early $\mathrm{T}$ dwarfs is detectable only with the largest telescopes because of the lack of continuum flux in this region. Also, in T dwarf atmospheres $\mathrm{LiCl}$ and $\mathrm{LiOH}$ become the dominant $\mathrm{Li}$ bearing molecule [Lodders 1999] and these species are currently undetectable.) Self-consistent evolutionary models accounting for each mechanism must be developed, as well as mechanisms to explain the onset of either patchiness or varying $f_{\text {sed }}$ at a particular $T_{\text {eff. }}$. We plan to more fully explore these issues in a future paper.

\subsubsection{Additional Considerations}

Discrepant objects to note in Figures 8 and 9 are Kelu-1 (L3), SDSS J0423-0414 (T0), and 2MASS J0415-0935 (T9). 2MASS J0415-0935 is significantly redder in $J-K$ than the models would predict, as discussed in $\S$ 5.6. Kelu-1 and SDSS J0423-0414 are both superluminous by about 0.75 mag, suggesting that they may be pairs of identical dwarfs in unresolved binary systems. Kelu-1 has been imaged by $H S T$ and by Keck, with no evidence of duplicity found (Martín et al. 1999a; Koerner et al. 1999), while SDSS J0423-0414 has not been imaged at high resolution to our knowledge. Cruz et al. (2003) classify this dwarf as L7.5 using red spectra; however, $\mathrm{CH}_{4}$ bands are clearly seen in the $\mathrm{G} 02$ spectrum (Fig. 3 in G02), and the bolometric correction (i.e., ratio of $K$-band flux to total luminosity) is more compatible with an infrared classification of $\mathrm{T}$ than $\mathrm{L}$ (Golimowski et al. 2004). Although it has been suggested that this object consists of a late $\mathrm{L}$ and early $\mathrm{T}$ close pair (Burgasser et al. 2003b), a discrepancy between the optical and infrared types is not unexpected. As described in $\S 5.2$, different wavelength regions probe different levels of the photosphere. It is likely that the optically derived spectral types are more representative of effective temperature, and as Golimowski et al. (2004) show that there is an apparent plateau at $T_{\text {eff }} \approx 1450 \mathrm{~K}$ for types L7 to T4, we would expect optical types to be earlier than infrared types for the $\mathrm{L}-\mathrm{T}$ transition objects. The earlier optical classification is in fact observed for six L8 to T0 dwarfs in our sample, as indicated in Table 9.

Although the clear atmosphere models do a good job of reproducing the colors of the later T dwarfs in the $J-H-H-K$ 
diagram (Fig. 6), they tend to predict bluer $J-K$ colors for these objects than is observed (Figs. 8 and 9). This seems to be a generic problem with clear models (see also Tsuji \& Nakajima 2003). The $J-K$ result implies that the model tracks shown in Figure 6 should slide up and to the right, consistent with the suggestion in $\S 5.6$ that the temperature contours are too warm. Because of the overall shape of the model contours, the quality of the fit in $J-H: H-K$ would remain about the same. The discussion of the gravity signature seen in $H-K$ is still qualitatively valid, although SDSS J1110+0116 would be expected to have a somewhat higher gravity.

Finally, we consider detectability limits for SDSS using Figures 3, 8, and 9. Many SDSS T dwarfs have to be selected as $z$-only objects and they are either not detected or only barely detected at $i$ (see Table 1 ). The nominal ( $5 \sigma$, better than 1 .'5 seeing) $z$-detection limit is 20.8 , and the faintest SDSS T dwarf discovered to date is close to this limit, at $z=20.4$. Since the colors of late (e.g., T8) dwarfs are $z-J \approx 3.8$, the corresponding $J$ limit is $\approx 16.6$; Table 9 therefore indicates that SDSS should be able to detect T9 dwarfs to $10 \mathrm{pc}$. The latest SDSS dwarf discovered to date is a T7, but we anticipate that some later types will be found.

\section{SUMMARY}

We have presented new near-infrared photometry and spectroscopy for cool dwarfs from two sources: new very red objects from SDSS and known L and T dwarfs from SDSS and 2MASS. We have obtained new $J H K$ photometry for 71 $\mathrm{L}$ and $\mathrm{T}$ dwarfs (53 from SDSS and 18 from 2MASS), $Z$ photometry for seven 2MASS objects, and spectroscopy of $56 \mathrm{~L}$ and $\mathrm{T}$ dwarfs (45 from SDSS and 11 from 2MASS). The spectral types have been obtained using the classification scheme of G02, which uses four molecular band indices at $J$, $H$, and $K$. The combined data from this and our previous papers are analyzed. Absolute magnitudes are available for 45 late-type dwarfs, thanks to recent parallax measurements. The relationships among color, absolute magnitude, and spectral type are compared with model atmospheres with and without clouds. The major results and conclusions are as follows:

1. Of the 44 new SDSS targets for which infrared spectra were obtained, one is an M dwarf, six are L dwarfs previously reported by Hawley et al. (2002) and Cruz et al. (2003), which are also classified as L in the G02 scheme, 23 are new L dwarfs, and 14 are new T dwarfs (one of which was classified as late L from optical spectra by Hawley et al. 2002). The new $\mathrm{T}$ dwarf sample consists of seven T0-T2 and seven T4.5-7 types, and we also identify nine L dwarfs with type L8 and later. These observations add significantly to the sample of L$\mathrm{T}$ transition objects and bring to 58 the total number of published $\mathrm{T}$ dwarf systems.

2. We provide provisional indices on the G02 scheme for the end of the T spectral sequence. The spectral type of 2MASS J0415-0935 is T9; it is currently the coolest known dwarf, with an effective temperature of $\sim 700 \mathrm{~K}$ (Golimowski et al. 2004; Vrba et al. 2004).

3. As recognized previously, the relatively muted colors of the L dwarfs (compared with models in which there is no sedimentation) imply that silicate and iron cloud optical depths are limited by condensate sedimentation.

4. As noted in previous work, the $J H K$ colors of mid to late L dwarfs show a large scatter within a given spectral type. The $J H K$ colors are reasonably reproduced by models that incorporate cloud formation with a modest range of condensate sedimentation efficiencies (or equivalently cloud optical depth). About $10 \%$ of the dwarfs in our sample seem to be either substantially bluer or redder than a modest range in $f_{\text {sed, }}$ of about 3 to 4, would predict. This suggests that cloud properties are generally similar, but can differ, among L dwarfs.

5. The reddest and bluest $\mathrm{L}$ dwarfs show a scatter in the infrared spectral indices, and there can also be significant differences between the spectral types determined using optical and near-infrared spectra. The differences between indices can be understood in terms of the depths probed by the different wavelength regions.

6. The near-infrared colors of $\mathrm{T}$ dwarfs become rapidly bluer toward later types. However, beyond about T5 these colors, especially $H-K$, show large scatter. This is correlated with the equivalent width of the $J$-band $\mathrm{K}$ I doublet absorption, strongly suggesting that the $H-K$ color is gravity-dependent. Model atmospheres show that the $K$-band flux is depressed by $\mathrm{H}_{2}$ absorption and that at a given effective temperature $H-K$ becomes bluer with increasing gravity. As intermediateage $\mathrm{T}$ dwarfs all have essentially the same radius, $H-K$ is a good indicator of mass for field $\mathrm{T}$ dwarfs of a given spectral type.

7. The implied masses of almost all of the $\mathrm{T}$ dwarfs in the sample are 15-75M Jupiter. The T5.5 dwarf SDSS J1110+0116 appears to have a particularly low mass $\left(10-15 M_{\text {Jupiter }}\right)$ with an inferred age of about $1-3 \times 10^{8} \mathrm{yr}$.

8. The absolute magnitude-spectral type relationship for $\mathrm{L}$ and $\mathrm{T}$ dwarfs shows a mostly steady decline toward later spectral type, from $M_{J} \sim 11.5, M_{K} \sim 10.5$ at L0 to $M_{J} \sim 16.5$, $M_{K} \sim 17$ at T9. There is a peak or plateau, depending on wavelength, in absolute magnitude between types L7 and T4 (where $T_{\text {eff }} \approx 1450 \mathrm{~K}$, Golimowski et al. 2004). Models (e.g., Burgasser et al. 2002b), that invoke an onset of some type of modification to the vertical or horizontal extent of the cloud at a fixed $T_{\text {eff }}$ seem to better explain this observation than models that assume continuous sinking of a cloud that is spatially and vertically uniform over time. Additional observational tests of the various cloud disruption possibilities are needed.

We are most grateful to the staff at UKIRT for their assistance in obtaining the data presented in this paper. Some data were obtained through the UKIRT Service Programme. UKIRT is operated by the Joint Astronomy Centre on behalf of the UK Particle Physics and Astronomy Research Council. D. A. G. thanks the Center for Astrophysical Sciences at Johns Hopkins University for its moral and financial support of this work. G. R. K. is grateful for support to Princeton University and to NASA via grants NAG5-8083 and NAG5-11094. M. S. M. acknowledges support from NASA grants NAG26007 and NAG5-8919 and NSF grant AST 00-86288. T. R. G.'s research is supported by the Gemini Observatory, which is operated by the Association of Universities for Research in Astronomy on behalf of the international Gemini partnership of Argentina, Australia, Brazil, Canada, Chile, the United Kingdom, and the United States. Funding for the creation and distribution of the SDSS Archive has been provided by the Alfred P. Sloan Foundation, the Participating Institutions, the National Aeronautics and Space Administration, the National Science Foundation, the Department of Energy, the Japanese Monbukagakusho, and the Max Planck Society. The SDSS 
Web site is http://www.sdss.org. The SDSS is managed by the Astrophysical Research Consortium for the Participating Institutions. The Participating Institutions are the University of Chicago, Fermilab, the Institute for Advanced Study, the Japan Participation Group, Johns Hopkins University, Los
Alamos National Laboratory, the Max Planck Institute for Astronomy, the Max Planck Institute for Astrophysics, New Mexico State University, University of Pittsburgh, Princeton University, the United States Naval Observatory, and the University of Washington.
Abazajian, K., et al. 2003, AJ, 126, 2081

Ackerman, A. S., \& Marley, M. S. 2001, ApJ, 556, 872

Allard, F., Guillot, T., Ludwig, H., Hauschildt, P. H., Schweitzer, A., Alexander,

D. R., \& Ferguson, J. W. 2003, in IAU Symp. 211, Brown Dwarfs, ed. E. Martín (San Francisco: ASP), 325

Allard, F., Hauschildt, P. H., Alexander, D. R., Tamanai, A., \& Schweitzer, A. 2001, ApJ, 556, 357

Becklin, E. E., \& Zuckerman, B. 1988, Nature, 336, 656

Beichman, C. A., Chester, T. J., Skrutskie, M., Low, F. J., \& Gillett, F. 1998, PASP, 110,480

Borysow, A., Jorgensen, U. G., \& Zheng, C. 1997, A\&A, 324, 185

Bouy, H., Brandner, W., Martín, E. L., Delfosse, X., Allard, F., \& Basri, G. 2003, AJ, 126, 1526

Burgasser, A. J., et al. 2002a, ApJ, 564, 421

- 2003a, ApJ, 592, 1186

Burgasser, A. J., Kirkpatrick, J. D., Liebert, J. \& Burrows, A. 2003b, ApJ, 594,510

Burgasser, A. J., Kirkpatrick, J. D., McElwain, M. W., Cutri, R. M., Burgasser, A. J., \& Skrutskie, M. F. 2003c, AJ, 125, 850

Burgasser, A. J., Kirkpatrick, J. D., Reid, I. N., Brown, M. E., Miskey, C. L., \& Gizis, J. E. 2003d, ApJ, 586, 512

Burgasser, A. J., Marley, M. S., Ackerman, A. S., Saumon, D., Lodders, K., Dahn, C. C., Harris, H. C., \& Kirkpatrick, J. D. 2002b, ApJ, 571, L151

Burgasser, A. J., McElwain, M. W., \& Kirkpatrick, J. D. 2003e, AJ, 126, 2487

Burrows, A., Burgasser, A. J., Kirkpatrick, J. D., Liebert, J., Milsom, J. A., Sudarsky, D., \& Hubeny, I. 2002, ApJ, 573, 394

Burrows, A., Hubbard, W. B., Lunine, J. I., \& Liebert, J. 2001, Rev. Mod. Phys., 73, 719

Burrows, A., Marley, M., Hubbard, W. B., Lunine, J. I., Guillot, T., Saumon, D., Freedman, R., Sudarsky, D., \& Sharp, C. 1997, ApJ, 491, 856

Burrows, A., Sudarsky, D., \& Lunine, J. I. 2003, ApJ, 596, 587

Burrows, A., \& Volobuyev, M. 2003, ApJ, 583, 985

Chabrier, G., \& Baraffe, I. 2000, ARA\&A, 38, 337

Collinge, M. J., et al. 2002, BAAS, 201, No. 16.03

Cruz, K. L., Reid, I. N., Liebert, J., Kirkpatrick, J. D., \& Lowrance, P. J. 2003, AJ, 126, 2421

Cushing, M. C., Rayner, J. T., Davis, S. P., \& Vacca, W. D. 2003, ApJ, 582, 1066

Dahn, C. C., et al. 2002, AJ, 124, 1170

Delfosse, X., et al. 1997, A\&A, 327, L25

Delfosse, X., Tinney, C. G., Forveille, T., Epchtein, N., Borsenberger, J., Fouqué, P., Kimeswenger, S., \& Tiphéne, D. 1999, A\&AS, 135, 41

Enoch, M. L., Brown, M. E., \& Burgasser, A. J. 2003, AJ, 126, 1006

Epchtein, N. 1997, in The Impact of Large Scale Near-IR Sky Surveys, ed.

F. Garzón, N. Epchtein, A. Omont, W. B. Burton, \& P. Persei (Dordrecht: Kluwer), 15

ESA. 1997, The Hipparcos and Tycho Catalogues (ESA SP-1200) (Noordwijk: ESA)

Fan, X., et al. 2000, AJ, 119, 928$$
\begin{aligned}
& 2001, \mathrm{AJ}, 122,2833 \\
& 2003, \mathrm{AJ}, 125,1649
\end{aligned}
$$

Fukugita, M., Ichikawa, T., Gunn, J. E., Doi, M., Shimasaku, K., \& Schneider, D. P. 1996, AJ, 111, 1748

Geballe, T. R., et al. 2002, ApJ, 564, 466 (G02)

Geballe, T. R., Saumon, D., Leggett, S. K., Knapp, G. R., Marley, M. S., \& Lodders, K. 2001, ApJ, 556, 373

Gelino, C. R., Marley, M. S., Holtzmann, J. A., Ackerman, A. S., \& Lodders, K. 2002, ApJ, 577, 433

Golimowski, D. A., et al. 2004, AJ, in press

Gorlova, N. I., Meyer, M. R., Rieke, G. H., \& Liebert, J. 2003, ApJ, 593, 1074

Gunn, J. E., et al. 1998, AJ, 116, 3040

Hall, P. B., et al. 2002, ApJS, 141, 267

Hawarden, T. G., Leggett, S. K., Letawsky, M. B., Ballantyne, D. R., \& Casali, M. M. 2001, MNRAS, 325, 563

Hawley, S. L., et al. 2002, AJ, 123, 3409

Hogg, D. W., Finkbeiner, D. P., Schlegel, D. J., \& Gunn, J. E. 2001, AJ, 122,2129

Jones, H. R. A., \& Tsuji, T. 1997, ApJ, 480, L39

Kirkpatrick, J. D., et al. 1999, ApJ, 519, 802
Kirkpatrick, J. D., et al. 2000, AJ, 120, 447

Koerner, D. W., Kirkpatrick, J. D., McElwain, M. W., \& Bonaventura, N. R. 1999, ApJ, 526, L25

Leggett, S. K., Allard, F., Geballe, T. R., Hauschildt, P. H., \& Schweitzer, A. 2001, ApJ, 548, 908

Leggett, S. K., et al. 2000, ApJ, 536, L35

. 2002a, ApJ, 564, 452

Leggett, S. K., Hauschildt, P. H., Allard, F., Geballe, T. R., \& Baron, E. 2002b, MNRAS, 332, 78

Liu, M. C., Wainscoat, R., Martín, E. L., Barris, B., \& Tonry, J. 2002, ApJ, 568, L107

Lodders, K. 1999, ApJ, 519, 793

Lucas, P. W., Roche, P. F., Allard, F., \& Hauschildt, P. H. 2001, MNRAS, 326,695

Lupton, R. H., Gunn, J. E., \& Szalay, A. S. 1999, AJ, 118, 1406

Lupton, R. H., Ivezić, Ž., Gunn, J. E., Knapp, G. R., Strauss, M. A., \& Yasuda, N. 2003, Proc. SPIE, 4836, 350

Marley, M. 2000, ASP Conf. Ser. 212, From Giant Planets to Cool Stars, ed. C. A. Griffith \& M. S. Marley (San Francisco: ASP), 152

Marley, M. S., Seager, S., Saumon, D., Lodders, K., Ackerman, A. S., Freedman, R. S., \& Fan, X. 2002, ApJ, 568, 335

Martín, E. L., Basri, G., Delfosse, X., \& Forveille, T. 1997, A\&A, 327, L29

Martín, E. L., Brandner, W., \& Basri, G. 1999a, Science, 283, 1718

Martín, E. L., Delfosse, X., Basri, G., Goldman, B., Forveille, T., \& Zapatero Osorio, M. R. 1999b, AJ, 118, 2466

Martín, E. L., Rebolo, R., \& Magazzu, A. 1994, ApJ, 436, 262

Martín, E. L., \& Zapatero Osorio, M. R. 2003, ApJ, 593, L113

Martín, E. L., Zapatero Osorio, M. R., Barrado y Navascués, D., Béjar, V. J. S., \& Rebolo, R. 2001, ApJ, 558, L117

McCaughrean, M. J., Close, L. M., Scholz, R.-D., Lenzen, R., Biller, B., Brandner, W., Hartung, M., \& Lodieu, N. 2004, A\&A, 413, 1029

McGovern, M. R., Kirkpatrick, J. D., McLean, I. S., Burgasser, A. J., Prato, L., \& Lowrance, P. J. 2004, ApJ, 600, 1020

McLean, I. S., Chuter, T. C., McCaughrean, M. J., \& Rayner, J. T. 1986, Proc. SPIE, 627, 430

McLean, I. S., McGovern, M. R., Burgasser, A. J., Kirkpatrick, J. D., Prato, L., \& Kim, S. S. 2003, ApJ, 596, 561

Nakajima, T., Oppenheimer, B. R., Kulkarni, S. R., Golimowski, D. A., Matthews, K., \& Durrance, S. T. 1995, Nature, 378, 463

Noll, K. S., Geballe, T. R., Leggett, S. K., \& Marley, M. S. 2000, ApJ, 541, L75

Oke, J. B., \& Gunn, J. E. 1983, ApJ, 266, 713

Perryman, M. A. C., et al. 1997, A\&A, 323, L49

Pier, J. R., Munn, J. A., Hindsley, R. B., Hennessy, G. S., Kent, S. M., Lupton, R. H., \& Ivezić, Z. 2003, AJ, 125, 1559

Ramsay-Howat, S. K., Ellis, M. A., Gostick, D. C., Hastings, P. R., Strachan, M., \& Wells, M. 2000, Proc. SPIE, 4008, 1067

Reid, I. N., Gizis, J. E., Kirkpatrick, J. D., \& Koerner, D. W. 2001, AJ, 121, 489

Roche, P. F., et al. 2003, Proc. SPIE, 4841, 901

Ruiz, M. T., Leggett, S. K., \& Allard, F. 1997, ApJ, 491, L107

Saumon, D., Bergeron, P., Lunine, J. I., Hubbard, W. B., \& Burrows, A. 1994, ApJ, 424, 333

Saumon, D., Marley, M. S., Lodders, K., \& Freedman, R. S. 2003, in IAU Symp. 211, Brown Dwarfs, ed. E. Martín (San Francisco: ASP), 345

Scholz, R.-D., McCaughrean, M. J., Lodieu, N., \& Kuhlbrodt, B. 2003, A\&A, 398, L29

Skrutskie, M. F., et al. 1997, in The Impact of Large Scale Near-IR Sky Surveys, ed. F. Garzón, N. Epchtein, A. Omont, B. Burton, \& P. Persei (Dordrecht: Kluwer), 25

Smith, J. A., et al. 2002, AJ, 123, 2121

Smith, V. V., et al. 2003, ApJ, 599, L107

Stephens, D. C. 2001, Ph.D. thesis, New Mexico State Univ.

- 2003, in IAU Symp. 211, Brown Dwarfs, ed. E. Martín (San Francisco: ASP), 355

Stephens, D. C., \& Leggett, S. K. 2004, PASP, 116, 9

Strauss, M. A., et al. 1999, ApJ, 522, L61

Tinney, C. G., Burgasser, A. J., \& Kirkpatrick, J. D. 2003, AJ, 126, 975

Tinney, C. G., Reid, I. N., Gizis, J., \& Mould, J. R. 1995, AJ, 110, 3014

Tsuji, T. 2002, ApJ, 575, 264

Tsuji, T., \& Nakajima, T. 2003, ApJ, 585, L151 
Tsvetanov, Z. I., et al. 2000, ApJ, 531, L61

van Altena, W. F., Lee, J. T., \& Hoffleit, E. D. 1995, The General Catalogue of Trigonometric Stellar Parallaxes (4th ed.; New Haven: Yale Univ. Obs.)

Volk, K., Blum, R., Walker, G., \& Puxley, P. 2003, IAU Circ. 8188, 2

Vrba, F. J., et al. 2004, AJ, in press
Wright, G. S., Mountain, C. M., Bridger, A., Daly, P. N., Griffin, J. L., \& Ramsay-Howat, S. K. 1993, Proc. SPIE, 1946, 547

York, D. G., et al. 2000, AJ, 120, 1579

Zapatero Osorio, M. R., Béjar, V. J. S., Martín, E. L., Rebolo, R., Barrado y Navascués, D., Mundt, R., Eislöffel, J., \& Caballero, J. A. 2002, ApJ, 578,536 